\title{
Geodesics of Random Riemannian Metrics
}

\author{
by \\ Tom LaGatta
}

A Dissertation Submitted to the Faculty of the Department of Mathematics In Partial Fulfillment of the Requirements

For the Degree of Doctor of Philosophy

In the Graduate College

The University of ARIZONA 


\section{THE UNIVERSITY OF ARIZONA GRADUATE COLLEGE}

As members of the Dissertation Committee, we certify that we have read the dissertation prepared by Tom LaGatta entitled

Geodesics of Random Riemannian Metrics

and recommend that it be accepted as fulfilling the dissertation requirement for the Degree of Doctor of Philosophy.

DATE: MAY 5, 2010

JAN WEHR

DATE: MAY 5, 2010

DAVID GLiCKEnSTEIN

DATE: MAY 5, 2010

Thomas G. Kennedy

DATE: MAY 5, 2010

Joseph C. WATKInS

FinAl APPROVAl AND ACCEPTANCE OF THIS DISSERTATION IS CONTINGENT UPON THE CANDIDATE'S SUBMISSION OF THE FINAL COPIES OF THE DISSERTATION TO the Graduate College.

I HEREBY CERTIFY THAT I HAVE READ THIS DISSERTATION PREPARED UNDER MY DIRECTION AND RECOMMEND THAT IT BE ACCEPTED AS FULFILLING THE DISSERTATION REQUIREMENT.

DATE: MAY 5, 2010

JAN WEHR 


\section{StATEMENT BY AUthoR}

THIS DISSERTATION HAS BEEN SUBMITTED IN PARTIAL FULFILLMENT OF REQUIREMENTS FOR AN ADVANCED DEgREE AT THE UNIVERSITY OF ARIZONA AND IS DEPOSITED IN THE UNIVERSITY LIBRARY TO BE MADE AVAILABLE TO BORROWERS UNDER RULES OF THE LIBRARY.

BRIEF QUOTATIONS FROM THIS DISSERTATION ARE ALLOWABLE WITHOUT SPECIAL PERMISSION, PROVIDED THAT ACCURATE ACKNOWLEDGMENT OF SOURCE IS MADE. REQUESTS FOR PERMISSION FOR EXTENDED QUOTATION FROM OR REPRODUCTION OF THIS MANUSCRIPT IN WHOLE OR IN PART MAY BE GRANTED BY THE HeAd of the Major Department or the Dean of the Graduate College WHEN IN HIS OR HER JUDGMENT THE PROPOSED USE OF THE MATERIAL IS IN THE INTERESTS OF SCHOLARSHIP. IN ALL OTHER INSTANCES, HOWEVER, PERMISSION MUST BE OBTAINED FROM THE AUTHOR.

Signed:

TOM LAGATTA 


\section{ACKNOWLEDGMENTS}

There is a long list of people I would like to thank who have helped get me through six years of graduate school. First and foremost, I thank my advisor Janek Wehr, whose passion and taste are what drew me to probability in the first place, and who has guided and supported me more than anyone else. I also thank the other members of my committee, Dave Glickenstein, Tom Kennedy and Joe Watkins, as well as Daniel Ueltschi, who have all been so generous with their advice, mathematical or otherwise. I thank Chuck Newman, who will be my supervisor at the Courant Institute next year, whose work has been a foundation for my own, and who has been so very generous with his time and help this past year.

I thank the members of the administration of the mathematics department at Arizona: Doug Ulmer, Ken McLaughlin and Tom Kennedy, the successive heads of the graduate program; Nick Ercolani and Bill McCallum, the successive heads of the department; and Michael Tabor, the current PI for the VIGRE grant. The math department would not be one tenth what it is without the excellent leadership of these men. I also thank the staff at the department, most notably Sandy Sutton, the departmental secretary for the graduate program, who single-handedly manages to keep at bay the tide of paperwork we graduate students have to face. Thanks also go out to the rest of the staff, including Sylvia Anderson, Jerrie Bieberstein, Bob Borys, Tina Deemer, David Gonzalez, Annette Horn, Denise Ingram, Anne Keyl, Christa King, Karl Newell, Alex Perlis, Karen Schaffner, Andrew Tubbiolo, Brooke Zang and every one else who has kept things running smoothly behind the scenes so that we grad students can focus on what we need to. I also thank the administration at the upper echelons of the College of Science, whose commitment to graduate student excellence is unshakable in these times of budget cuts and transformations: Joaquin Ruiz, Elliott Cheu, and Gail Burd, and their outstanding staff members including Danielle Shirar and Bernadette Thomas.

There are many great teachers at Arizona, and I have been lucky to have worked with many of them. I thank Bruce Bayly, Vita Borovyk, Sunhi Choi, Scott Clark, Carl Devito, Lennie Friedlander, Donna Krawczyk, Doug Pickrell, Natalie Sandler, Deidre Smith, Laurie Varecka, Mariamma Varghese, Bill Vélez, Joe Watkins and Janek Wehr, who have all helped shape me into a better teacher.

My research has been supported for many semesters under the NSF VIGRE grant at the University of Arizona (DMS-06-02173), and for one semester under NSF grant DMS-06-23941. I thank Krzysztof Gawȩdzki and ENS-Lyon for being outstanding hosts while I was in Lyon for a semester.

I am thankful for the Epic Café, Caffè Luce and Grill in Tucson, where I spent countless hours in graduate school working, enjoying life, then working some more. I thank my many, many friends in Tucson, San Francisco, New York City, Austin, 
Vancouver, Lyon and other cities across the globe, who continue to remind me how beautiful and diverse life can be, and how much opportunity it has to offer. In this list I include my lovely officemates Jordan Allen-Flowers, Josh Chesler, Yaron Hadad, Jeffrey Hyman, Selin Kalaycioglu, Anya Petersen, Mandi Schaeffer Fry, Brad Weir, and Mei Yin, as well as my best friend Ben Polletta and my girlfriend Emily Chambliss.

Finally and most importantly, I thank my parents, Barbara and Tom LaGatta. I have reached this point only by standing on their shoulders. 


\title{
Dedication
}

\author{
To Tucson.
}




\section{TABle of Contents}

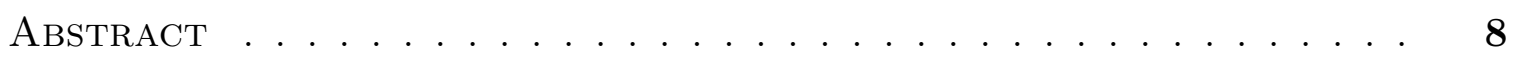

Chapter 1. Introduction . . . . . . . . . . . . . 9

1.1. Standard First-Passage Percolation _. . . . . . . . . . . . . . . 12

1.1.1. Time Constant . . . . . . . . . . . . . . . . . . . 12

1.1.2. Shape Theorem . . . . . . . . . . . . . . . . . 13

1.1.3. Shape Fluctuations and $\chi \ldots \ldots \ldots \ldots \ldots$

1.1.4. Transversal Fluctuations and $\xi \ldots \ldots \ldots$

1.1.5. Geodesics and Disordered Ferromagnets . . . . . . . . . 18

1.2. Other Models Related to First-Passage Percolation . . . . . . . . . 21

1.2.1. Euclidean First-Passage Percolation . . . . . . . . . . . 22

1.2.2. Last-Passage Percolation . . . . . . . . . . . . . . . . . . 24

1.2.3. Directed Polymers in a Random Environment . . . . . . . . . 25

1.2.4. Spin Glasses . . . . . . . . . . . . . . . . . . . . . . . . . . . 29

1.3. Included Papers . . . . . . . . . . . . . . . . . . . . . . 31

Chapter 2. Present Study . . . . . . . . . . . . . . 33

2.1. Geometry Background and Notation . . . . . . . . . . 34

2.2. Riemannian FPP . . . . . . . . . . . . . . . 35

2.3. The evolution of the environment under the geodesic flow . . . . . 37

2.4. Rarity of Minimizing Geodesics . . . . . . . . . . . . . 38

2.5. Transience of Geodesics and Existence of Frontier Times . . . . . . 41

2.6. Uniform Probability Estimates at Frontiers . . . . . . . . . . . . 44

2.7. Construction of a Bump Surface at Frontier Times and Proof of Main Result . . . . . . . . . . . . . . . . . . 48

Chapter 3. Proof of Theorem $2.10 \ldots \ldots \ldots \ldots \ldots \ldots \ldots$

Chapter 4. Sketch of Proof of Claim $2.14 \ldots \ldots \ldots \ldots . \ldots 1$

REFERENCES ............................ 69

Appendix A. A Shape Theorem for Riemannian First-Passage Per-

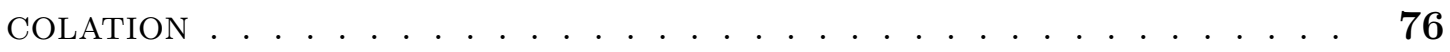

Appendix B. Continuous Disintegrations of Gaussian Measures . 104 


\begin{abstract}
We introduce Riemannian First-Passage Percolation (Riemannian FPP) as a new model of random differential geometry, by considering a random, smooth Riemannian metric on $\mathbb{R}^{d}$. We are motivated in our study by the random geometry of first-passage percolation (FPP), a lattice model which was developed to model fluid flow through porous media. By adapting techniques from standard FPP, we prove a shape theorem for our model, which says that large balls under this metric converge to a deterministic shape under rescaling. As a consequence, we show that smooth random Riemannian metrics are geodesically complete with probability one.

In differential geometry, geodesics are curves which locally minimize length. They need not do so globally: consider great circles on a sphere. For lattice models of FPP, there are many open questions related to minimizing geodesics; similarly, it is interesting from a geometric perspective when geodesics are globally minimizing. In the present study, we show that for any fixed starting direction $v$, the geodesic starting from the origin in the direction $v$ is not minimizing with probability one. This is a new result which uses the infinitesimal structure of the continuum, and for which there is no equivalent in discrete lattice models of FPP.
\end{abstract}




\section{Chapter 1 INTRODUCTION}

Standard first-passage percolation (Standard FPP) is a model of random geometry on the discrete lattice, famously introduced by Hammersley and Welsh [HW65] in order to model fluid flow through porous media. The model is simple to define: take the lattice $\mathbb{Z}^{d}$ and associate to each bond (edge) a random number, called the passage time. This induces a random metric on $\mathbb{Z}^{d}$, where the distance between two points is the infimum of passage times over all paths which connect the two points. Our model, Riemannian first-passage percolation, is a continuum analogue of Standard FPP. Instead of a random discrete metric on the lattice $\mathbb{Z}^{d}$, we consider a random Riemannian metric in the continuum $\mathbb{R}^{d}$, which again gives rise to a random distance function. Both the lattice and continuum models have a similar global geometric structure, but Riemannian geometry provides a rich local structure to our model.

Our consideration of a random Riemannian metric is a novel approach not found in the differential geometry literature. For large-scale properties which do not depend on the local structure of the metric, we are able to directly adapt techniques from Standard FPP to our model. To do this, we discretize the plane into unit cubes, and consider a dependent FPP model on the lattice formed by their centers. We exploit this strategy in proving a shape theorem in our article [LW10], included in Appendix A: large balls under this metric converge to a deterministic shape under rescaling. We also show that the metric is almost surely geodesically complete.

In this dissertation, we sketch a proof of a new result which exploits the infinitesimal structure of our model, and which is not available for lattice models. We assume now that our random metric has a rotationally invariant distribution, so that the limiting shape is a Euclidean ball. As in differential geometry [Lee97], we define 
geodesics to be curves which locally minimize arc length under our Riemannian metric. Geodesics need not globally minimize length: on the sphere, for example, geodesics are great circles, which do not minimize length past antipodal points. Geodesics are defined by a local condition: given a point and a direction, we define a geodesic to be the solution to a certain ordinary differential equation. The completeness of the metric guarantees that geodesics can be extended for all time. Geodesics can also be defined by a global condition as the curves which minimize the distance between two points, though in that case they need not be unique (consider the geodesics which connect antipodal points on a sphere).

Our main result is that globally length-minimizing geodesics are rare, and the following event holds with probability one: starting at the origin, the set $\mathcal{V} \subseteq S^{d-1}$ of directions which results in minimizing geodesics has Lebesgue measure zero on the sphere. This measure-zero property is not a technicality: the set $\mathcal{V}$ is non-empty and we furthermore conjecture that it is uncountable. We believe our proof of the main result is correct, though we still have some technical details to finish and plan to submit it for publication soon.

The proof of the main result is detailed and is split into three separate sections in Chapter 2. We consider the geodesic $\gamma$ starting at the origin in a fixed direction $v \in S^{d-1}$. By adapting our techniques from [LW10], we show in Section 2.5 that there is a sequence of "frontier times" $t_{k}$ along the geodesic at which the metric is "well-behaved" in a neighborhood $B_{k}$ of $\gamma\left(t_{k}\right)$. As the geodesic exists the Euclidean ball $B^{\mathrm{E}}\left(0,\left|\gamma\left(t_{k}\right)\right|\right)$, being well-behaved means that its exit velocity satisfies a cone condition, and the geodesics are bounded uniformly away from being tangential to the ball. The metric is well-behaved in the sense that we have a uniform bound on the $C^{2+\alpha}$-norm of $g$ in $B_{k}$, as well as a lower bound for the minimum eigenvalue of $g$ in $B_{k}$.

In Section 2.6, we show that there is a uniform lower bound $p>0$ of the probability that a certain event $U_{k}$ occurs at the frontier time $t_{k}$. In the proof, we change 
perspectives from the FPP context and focus on probability measures on Banach spaces, by means of a Strong Markov Property. The Arzelà-Ascoli theorem [Fol99] implies that the set $\Gamma$ of well-behaved metrics on $B_{k}$ is compact in the space of $C^{2}$ metrics. We show that conditional probabilities in this context vary continuously on the conditioning, so by minimizing over the compact set $\Gamma$ we have a positive lower bound on the probability of $U_{k}$. In order to carry out this uniform probability argument, the author developed the concept of continuous disintegrations, which evolved into the separate publication [LaG10], included in Appendix B.

It is the presence of positive curvature which destabilizes minimizing geodesics [LRST03]. In Section 2.7, we exploit this observation, and we describe a way to extend the metric at $\gamma_{v}\left(t_{k}\right)$ to a bump metric ahead, and argue that geodesics cannot be minimizing after spending enough time on the bump (like the top two-thirds of a sphere). This property is perturbed under small perturbations of the bump metric, so the event $U_{k}$ is that the random metric $g$ is sufficiently close to the bump metric in the region ahead.

Finally, we put the pieces together to prove the main result. We fix a direction $v \in S^{d-1}$, and estimate the probability that $v \in \mathcal{V}$ (i.e. that the geodesic $\gamma$ which it generates is minimizing). If there is not a sequence of frontier times $t_{k}$ as described above, the geodesic is not minimizing; supposing there is such a sequence, at each $t_{k}$ there is a uniform probability $p$ that the geodesic runs over a bump and stops being minimizing. Consequently, with probability one, $v \notin \mathcal{V}$.

For the remainder of this Introduction, we give a review of the literature for Standard FPP (Section 1.1) and related models (Section 1.2). In Section 1.3, we described the articles which we include in Appendices A and B. 


\subsection{Standard First-Passage Percolation}

We formally introduce the model of Standard FPP. Consider the $d$-dimensional lattice $\mathbb{Z}^{d}$ with $d \geq 2$. Let $\left\{t_{b}\right\}$ be a family of independent, identically distributed, nonnegative random variables, indexed by bonds (nearest-neighbor edges) $b$ of the lattice. For any $z, z^{\prime} \in \mathbb{Z}^{d}$, define the passage time from $z$ to $z^{\prime}$ by

$$
\tau\left(z, z^{\prime}\right)=\inf _{\gamma} \sum_{b \in \gamma} t_{b},
$$

where the infimum is taken over all lattice paths $\gamma$ connecting $z$ to $z^{\prime}$. This $\tau$ is a random distance function on $\mathbb{Z}^{d}$. For a very good introduction to Standard FPP, see Howard [How04] or the more recent Blair-Stahn [BS10].

\subsubsection{Time Constant}

The first object of study is the passage time $a_{n}:=\tau\left(0, n \mathrm{e}_{1}\right)$ between the origin and the point $n \mathrm{e}_{1}=(n, 0, \ldots, 0)$. One wishes to study the asymptotic behavior of this quantity as $n \rightarrow \infty$. In [Kin68], Kingman formulated his famous subadditive ergodic theorem in order to prove the basic result of FPP:

Theorem 1.1. If the passage times have finite mean, there exists a non-random constant $\mu_{\mathrm{e}_{1}}$, such that

$$
\lim _{n \rightarrow \infty} \frac{1}{n} \tau\left(0, n \mathrm{e}_{1}\right)=\mu_{\mathrm{e}_{1}}
$$

almost surely and in $L^{1}$.

By symmetry, the same value is the limit for the passage times $\frac{1}{n} \tau\left(0, n \mathrm{e}_{i}\right)$ in any of the coordinate directions $\mathrm{e}_{i}$. More generally, for each direction $v \in S^{d-1}$, there exists a non-random constant $\mu_{v}$, such that

$$
\lim _{n \rightarrow \infty} \frac{1}{n} \tau(0, \widetilde{n v})=\mu_{v}
$$


almost surely and in $L^{1}$, where $\widetilde{n v} \in \mathbb{Z}^{d}$ is the nearest lattice point to $n v$. The constants $\mu_{v}$ vary continuously with $v$ (cf. [LW10, Proposition 1.3]). Kesten [Kes84] has shown that the constant $\mu_{v}$ is non-zero provided that the probability that $t_{b}=0$ is less than the critical percolation probability for $\mathbb{Z}^{d}$; see [How04] for more details. While Kingman's theorem asserts the existence of the time constants $\mu_{v}$, they have not been computed explicitly for any non-trivial distribution of passage times for Standard FPP.

\subsubsection{Shape Theorem}

Henceforth, we assume that $\mu_{v}>0$ for all $v \in S^{d-1}$, and that the passage time distribution satisfies the simple moment condition

$$
\mathbb{E} \min \left\{t_{1}, \ldots, t_{2 d}\right\}^{2 d}<\infty
$$

for $2 d$ independent copies $t_{1}, \cdots, t_{2 d}$ of $t_{b}$. Where (1.1) is a law of large numbers-type statement for each fixed direction $v$, the shape theorem of Cox and Durrett [CD81] is a stronger result which holds for all directions simultaneously. We extend the function $\mu_{v}$ to a norm on $\mathbb{R}^{d}$ by defining $\mu(x):=\mu_{x /|x|}|x|$. Consider the unit ball in this norm,

$$
A=\{x: \mu(x) \leq 1\}=\left\{x:|x| \leq \mu_{x /|x|}^{-1}\right\}
$$

This non-random set depends only on the distribution of the passage times $t_{b}$, and is convex, compact, and invariant under the symmetries of the lattice $\mathbb{Z}^{d}$.

Consider

$$
\tilde{B}_{t}=\left\{z \in \mathbb{Z}^{d}: \tau(0, z) \leq t\right\}
$$

the random ball of radius $t$ in $\mathbb{Z}^{d}$. This is a lattice object, so we "inflate" it to get a continuum one: for $z \in \mathbb{Z}^{d}$, let $C_{z}=[z-1 / 2, z+1 / 2)^{d}$ be the unit cube centered at $z$ in $\mathbb{R}^{d}$, and let

$$
B_{t}=\bigcup_{z \in \tilde{B}_{t}} C_{z} .
$$


We define the rescaling $\frac{1}{t} B_{t}$ as the set of all points $x \in \mathbb{R}^{d}$ such that $t x \in B_{t}$. The shape theorem says essentially that $\frac{1}{t} B_{t} \rightarrow A$ :

Theorem 1.2. For all $\epsilon>0$, with probability one, there exists a time $T$ such that if $t \geq T$, then

$$
(1-\epsilon) A \subseteq \frac{1}{t} B_{t} \subseteq(1+\epsilon) A
$$

While the existence of a limiting shape $A$ is guaranteed by this theorem, it is in practice and in theory very difficult to obtain much information on the precise shape A. For Standard FPP, there are no known passage-time distributions which yield a rotationally-invariant limiting shape-lattice effects always seem to persist [How04]. Durrett and Liggett [DL81] have shown that if the distribution of $t_{b}$ has a positive atom with sufficiently high probability, then there are "facets" in the limiting shape: where it meets the diagonal directions, $\partial B$ is made up of flat pieces.

One expects that the facets of Durrett and Liggett are pathological, and that the boundary $\partial A$ should typically satisfy some smoothness properties. Newman and Piza [NP95] define a direction of curvature $v \in S^{d-1}$ if $\partial A$ is locally spherical near $x:=v / \mu_{v} \in \partial A$. Precisely, this means that there exists a Euclidean ball $D$ depending on the direction $v$ which contains $A$ and is tangent to $A$ at $x$ :

$$
A \subseteq D \text { and } x \in \partial D
$$

There is a simple proof that directions of curvature exist in Standard FPP: let $r$ be the minimal radius such that the Euclidean ball $D=B(0, r)$ centered at the origin contains $A$, then the directions where $D$ meets $A$ are directions of curvature [How04]. This is a very weak existence result, however, and there may only be finitely many directions of curvature. Moreover, no specific direction has been verified to be a direction of curvature for any distribution of passage times, including the axial directions [How04]. 
Newman [New95] says that $A$ is uniformly curved if every direction is a direction of curvature, and moreover that the radii of the balls $D$ is uniformly bounded away from infinity. In the $d=3$ case where $\partial A$ is a topological 2-sphere, this assumption is that the Gaussian curvature is uniformly bounded away from 0 on the boundary surface $\partial A$. Again, this has not been verified for any particular distribution of passage times in Standard FPP. However, in the rotationally invariant models of Euclidean FPP of Newman and Howard [HN97] and Riemannian FPP of LaGatta and Wehr [LW10], the limiting shape is a Euclidean ball. Consequently, all directions are directions of curvature, and the limiting shape is uniformly curved.

\subsubsection{Shape Fluctuations and $\chi$}

As is to be expected from a law of large numbers, the upper bound $\epsilon t$ in (1.3) on the fluctuations of $B_{t}$ from $t A$ is far from optimal. Using an exponential moment condition, Kesten [Kes93] was able to improve (1.3) to

\section{Theorem 1.3.}

$$
\left(t-c t^{\kappa} \log t\right) A \subseteq B_{t} \subseteq\left(t+c t^{1 / 2} \log t\right) A
$$

for some non-random constant $c>0$ and $\kappa<1$.

In his proof, he also showed that given a second-moment condition, the variance of the passage time $a_{n}=\tau\left(0, n \mathrm{e}_{1}\right)$ was at worst linear:

$$
\operatorname{Var} a_{n} \leq C n,
$$

for some non-random constant $C>0$.

Let us define the longitudinal fluctuation exponent $\chi$ as the minimum number $k$ such that, with probability one, there exists a time $T$ such that if $t \geq T$, then

$$
\left(t-t^{k}\right) A \subseteq B_{t} \subseteq\left(t+t^{k}\right) A
$$


Alexander [Ale93, Ale97] was able to improve Kesten's estimate and proved (1.5) with the value $k=1 / 2$. Consequently, in terms of the fluctuation exponent this is the upper bound $\chi \leq 1 / 2$.

The above discussion may suggest that $a_{n}$ behaves diffusively. In fact, this is far from the case. First-passage percolation is conjectured to lie in the Kardar-ParisiZhang (KPZ) universality class of growth processes [KPZ86, KS88, KS91]. In twodimensions, the optimal value of the fluctuation exponent $\chi$ should be $1 / 3$, and it is believed that the variance $\operatorname{Var} a_{n}$ is of order $n^{2 / 3}$. We will explore this connection in more detail in the next section.

Benjamini, Kalai and Schramm [BKS03] used the concentration inequalities of Talagrand [Tal94] in order to show that $a_{n}$ has sublinear distance variance

$$
\operatorname{Var} a_{n} \leq C n / \log n
$$

for a Bernoulli distribution of passage times, and Benaïm and Rossignol [BR06, BR08] were able to extend this to a much wider class of passage time distributions, including exponential passage times. This may seem like a trivial improvement of (1.4), but in fact is quite significant. Chatterjee [Cha08] has some remarkable applications of sublinear distance variance (or "superconcentration" in his terminology) which have not yet been applied successfully to first-passage percolation; he has, however, done this for spin-glass models [Cha09], and we will discuss this more in Section 1.2.4.

\subsubsection{Transversal Fluctuations and $\xi$}

If the passage time distribution does not have any atoms, then with probability one, for all $n$ there exists a unique path $\gamma_{n}$ which realizes the minimum passage time $a_{n}=\tau\left(0, n \mathrm{e}_{1}\right)$ from the origin to $n \mathrm{e}_{1}[\mathrm{How} 04]$. Let $d_{n}$ be the maximal distance that $\gamma_{n}$ deviates from the straight line path from 0 to $n \mathrm{e}_{1}$. Formally,

$$
d_{n}=\sup \left\{\inf _{0 \leq j \leq n}\left|\gamma_{n}(i)-j \mathrm{e}_{1}\right|: 0 \leq i \leq\left|\gamma_{n}\right|\right\},
$$


where $\left|\gamma_{n}\right|$ is the number of points in the path $\gamma_{n}$, and $\left|\gamma_{n}(0)-j \mathrm{e}_{1}\right|$ is the Euclidean distance between the points $\gamma_{n}(i)$ and $j \mathrm{e}_{1}$ in $\mathbb{Z}^{d} \subseteq \mathbb{R}^{d}$.

We define the transversal fluctuation exponent $\xi$ as the minimum number such that with probability one,

$$
d_{n}=O\left(n^{\xi}\right)
$$

We can similarly define the exponent $\xi(v)$ in the direction $v \in S^{d-1}$, though it is believed that the quantities $\xi(v)$ are invariant under direction. As mentioned in the previous section, it is conjectured that first-passage percolation lies in the KPZ universality class of growth processes [KS91], and the fluctuation exponents $\chi$ and $\xi$ satisfy the KPZ equation

$$
\chi=2 \xi-1 .
$$

It is further conjectured in dimension $d=2$ that $\chi=1 / 3$ and $\xi=2 / 3$ [KS91].

In terms of rigorous results, Newman and Piza [NP95] have partially proved an inequality of the form (1.6), but only as an inequality, and for transversal fluctuations in directions of curvature:

Theorem 1.4. If $v$ is a direction of curvature,

$$
\chi \geq 2 \xi(v)-1 .
$$

Along with the Kesten-Alexander upper bound $\chi \leq 1 / 2$, this implies the upper bound $\xi(v) \leq 3 / 4$ on transversal fluctuations in directions of curvature. Using techniques based on Wehr-Aizenman [WA90], Newman and Piza [NP95] are also able to prove the lower bound

$$
2 \chi^{\prime}(v) \geq 1-(d-1) \xi(v)
$$

for an exponent $\chi^{\prime}(v)$ related to $\chi$. They conjecture that $\chi^{\prime}(v)$ is independent of direction and is in fact equal to $\chi$.

If the KPZ equation (1.6) holds, then the trivial bound $\chi \geq 0$ implies that $\xi \geq 1 / 2$. This is non-trivial, since the value $\xi=1 / 2$ corresponds to the process $d_{n}$ behaving 
diffusively (e.g. like a simple random walk). However, it is believed that $d_{n}$ behaves super-diffusively, and $\xi>1 / 2$. Under some weak conditions, Licea, Newman and Piza [LNP96] rigorously prove the lower bound $\xi \geq 1 /(d+1)$ for all dimensions $d$, as well as $\xi^{\prime}(d) \geq 1 / 2$ for a related exponent $\xi^{\prime}(d)$ depending on dimension $d$, and $\xi^{\prime}(2) \geq 3 / 5>1 / 2$. It is conjectured that $\xi^{\prime}=\xi$, but it is still an open question to prove rigorously that $\xi>1 / 2$ for any model of FPP.

\subsubsection{Geodesics and Disordered Ferromagnets}

As evidenced from the above section, minimizing paths are of critical important to the study of first-passage percolation: the fluctuations of minimizing paths are related to the fluctuations of the limiting shape via the KPZ equation (1.6). Assume that the passage time distributions are continuous. This implies that finite minimizing paths exist; however, the existence of infinite minimizing paths is a subtler question.

In the first-passage percolation literature, minimizing paths are denoted by the term "geodesic." This is very different from the standard meaning of the word in differential geometry. As we will discuss in more detail in Chapter 2, geodesics are curves which locally minimize length, but not necessarily globally. On the sphere, for example, geodesics are great circles, which do not globally minimize distance past antipodal points. In this section, we only use the term geodesic to refer to minimizing lattice paths, since there is not an infinitesimal notion of geodesic for lattice models. However, when we discuss the model of Riemannian FPP in Chapter 2, we will distinguish between "geodesics" which only locally minimize length, and "minimizing geodesics" which do so globally.

We say that a path $\gamma: \mathbb{N} \rightarrow \mathbb{Z}^{d}$,

$$
\gamma=\left(\gamma^{0}, \gamma^{1}, \gamma^{2}, \ldots\right)
$$

is a one-sided geodesic if for every pair of points $x, y \in \gamma$, the passage time $\tau(x, y)$ is realized as the passage time along $\gamma$. Provided that the passage time distribution has 
no atoms, between any two points there exists a unique minimizing path [How04]. It is easy to extend this using a spanning-tree argument to show that with probability one, for every point $z \in \mathbb{Z}^{d}$, there exists a one-sided geodesic starting at $z$. Fix $z$, and for every point $z^{\prime}$ let $\gamma_{z^{\prime}}$ denote the unique minimizing path connecting $z$ and $z^{\prime}$. Let

$$
\mathcal{T}(z)=\bigcup_{z^{\prime}} \gamma_{z^{\prime}}
$$

be the union of the edges of these minimizing paths. Clearly, $\mathcal{T}(z)$ is a spanning tree of $\mathbb{Z}^{d}$ hence contains an infinite path starting at $z$.

The above demonstrates that there is at least one geodesic at each point, though Newman [New95] conjectures that there should be infinitely many. For $w \in S^{d-1}$, we say that $w$ is an asymptotic direction for a geodesic if the limit

$$
\lim _{n \rightarrow \infty} \frac{\gamma^{n}}{\left|\gamma^{n}\right|}
$$

exists and equals $w$. Under the assumption of uniform curvature on the limiting shape, Newman shows that, with probability one, every one-sided geodesic at the origin has an asymptotic direction. Furthermore, every direction $w \in S^{d-1}$ is realized as the asymptotic direction for at least one geodesic, which implies that there are infinitely many geodesics at the origin. While the uniform curvature assumption is not satisfied for any known distribution of passage times, these arguments have been successfully applied to other models, which we discuss more in Section 1.2.

For Standard FPP, Häggström and Pemantle [HP98] are able to show that if $d=2$ and the passage times have an exponential distribution, then with positive probability any particular site (e.g. the origin) has at least two distinct one-sided geodesics. Their argument involves a connection to Richardson's growth model [Ric73]. Hoffman [Hof08] extends their ideas to show that the number of one-sided geodesics at the origin is at least 4 with positive probability. The number 4 comes from the minimum number of sides of the limiting shape $A$ in $d=2$. If $A$ is not polygonal (for example a ball), then we say it has infinitely-many sides, and accordingly the number 4 is 
improved to $\infty$. Unfortunately, like the uniform curvature assumption, that $A$ is not polygonal has not been rigorously shown for any passage time distribution.

We extend the definition of geodesic to two-sided paths $\gamma: \mathbb{Z} \rightarrow \mathbb{Z}^{d}$,

$$
\gamma=\left(\ldots, \gamma^{-1}, \gamma^{0}, \gamma^{1}, \ldots\right)
$$

and say that $\gamma$ is a two-sided geodesic if for every pair of points $x, y \in \gamma$, the passage time $\tau(x, y)$ is realized as the passage time along $\gamma$. The existence of two-sided geodesics is an important open question, and it is believed that the answer is different for dimensions $d=2$ and $d>2$. In either case, Wehr [Weh97] has shown that if twosided geodesics do exist, then there are infinitely many of them with probability one.

The existence of two-sided geodesics has consequences for statistical physics. In $d=2$, Standard FPP is essentially the dual model for a disordered ferromagnet, a simplification of the Edwards-Anderson spin glass [EA75] where the nearest-neighbor couplings are non-negative random variables [New97]. Consequently, the almost-sure existence of two-sided geodesics is equivalent to the almost-sure existence of nontrivial ground states in this model. For physical reasons, it is conjectured that these do not exist. We will discuss spin-glass models more in Section 1.2.4; for more details on the connection between these two models, see Newman [New97].

Under the assumption of uniform curvature on the limiting shape $A$, Newman [New95] shows that the only two-sided geodesics which can exist are those with antipodal asymptotic directions. i.e., with probability one, for each two-sided geodesic $\gamma$ there exists $w \in S^{d-1}$ such that

$$
\lim _{n \rightarrow \pm \infty} \frac{\gamma^{n}}{\left|\gamma^{n}\right|}= \pm w .
$$

In the $d=2$ case, Licea and Newman [LN96] show that for each deterministic $w$ that, with probability one, there does not exist a two-sided geodesic with asymptotic directions $w$ and $-w$. Their argument is to fix $w \in S^{d-1}$, and consider the minimizing paths $\gamma_{n}$ from $-n w$ to $n w$ (properly adjusted so the points $\pm n w$ lie on the lattice). If 
a two-sided geodesic $\gamma$ with asymptotic directions $\pm w$ were to exist, then $\gamma_{n}$ should converge to it; if $d_{n}$ equals the minimal distance from the origin to $\gamma_{n}$, then $d_{n}$ is of order 1 . However, by Section 1.1.4, $d_{n}$ should scale like $n^{\xi}$, so a positive lower bound on $\xi$ gives a contradiction.

One of the few solid non-existence results is due to Wehr and Woo [WW98], who have shown that for FPP restricted to the half-lattice in $d=2$, there exist no two-sided geodesics with probability one.

There is a heuristic scaling argument that suggests superdiffusivity of transversal fluctuations (i.e. $\xi>1 / 2$ ) implies non-existence of two-sided geodesics in 2dimensional Standard FPP [New10]. Consider a circle of large radius $R$ centered at the origin, and break it into arcs of length $O\left(R^{\xi}\right)$, so that there are $O\left(R^{1-\xi}\right)$ such $\operatorname{arcs} \alpha_{i}$. If two-sided geodesics exist with probability one, then one meets the origin with some probability $p>0$ not depending on $R$. By coalescence arguments [LN96, HN97], for all points $x \in \alpha_{i}$ and $y \in-\alpha_{i}$ on antipodal arcs, the minimizing paths from $x$ to $y$ should all coalesce with high probability. Thus there is essentially only one geodesic passing between antipodal arcs, whose transversal fluctuations are of order $O\left(R^{\xi}\right)$ by definition of $\xi$; consequently, the probability that it passes through the origin is of order $O\left(R^{-\xi}\right)$. Roughly speaking, for different arcs, these events are almost independent, so by considering them as Bernoulli trials, the probability of at least one occurring is $O\left(R^{1-\xi} \cdot R^{-\xi}\right)=O\left(R^{1-2 \xi}\right)$. If $\xi>1 / 2$, this probability goes to zero; in particular, for large $R$ it is less than $p$, a contradiction.

\subsection{Other Models Related to First-Passage Percolation}

Boivin [Boi90] introduced a model of stationary, ergodic FPP, where one considers $d$ different passage time distributions, one for each direction $\mathrm{e}_{1}, \ldots, \mathrm{e}_{d}$, and where the assumption of independence of passage times is relaxed to ergodicity. He shows that a shape theorem is satisfied, and that the limiting shape $A$ in this setting need only 
be compact, convex and satisfy the antipodal symmetry $A=-A$. Häggström and Meester [HM95] show that these conditions on $A$ are sufficient for there to exist a stationary, ergodic passage time distribution with limiting shape $A$. Consequently, there exists some distribution of stationary, ergodic passage times for which $A$ is a Euclidean ball.

Chatterjee-Dey [CD09] consider a model of first-passage percolation restricted to growing cylinders of the form

$$
[0, n] \times\left[-h_{n}, h_{n}\right] \subseteq \mathbb{Z}^{2}
$$

where $h_{n}=o\left(n^{1 / 3}\right)$ (as well as the generalization to higher dimensions). In this context, they show that the first passage times $a_{n}=\tau\left(0, n \mathrm{e}_{1}\right)$ satisfy a Gaussian central limit theorem; in particular, the fluctuations of $a_{n}$ are order of $\sqrt{n}$. This is qualitatively different than the expected behavior in Standard FPP, where it is believed that $a_{n}$ has fluctuations of order $n^{\chi}$, and does not satisfy a Gaussian central limit theorem. Thus this is some rigorous evidence suggesting the lower bound $\chi \geq$ $1 / 3$ for Standard FPP.

\subsubsection{Euclidean First-Passage Percolation}

In Standard FPP, lattice effects always seem to persist at the macroscopic scale: the limiting shape $A$ is not rotationally-invariant for any known passage time distribution. As we saw in the last section, this is a major obstruction, as many results for Standard FPP on fluctuation exponents or the existence of geodesics hold only under strict curvature assumptions on the limiting shape. In order to circumvent this rigidity, Vahidi-Asl and Wierman [VAW90, VAW92] consider a model of FPP on the Voronoi graph generated by the points of a random Poisson point process $Q$ in $\mathbb{R}^{d}$. They are able to prove a shape theorem and, since $Q$ has a rotationally-invariant distribution, the limiting shape is a Euclidean ball. The shape theorem for this model is nontrivial for any distribution of passage times - including the case when passage times 
are non-random and constant — as the Voronoi graph of a random point process may be quite complicated.

Howard and Newman [HN97] consider another model based on a Poisson point process $Q$ in $\mathbb{R}^{d}$, which they term Euclidean FPP. Rather than deal with the complicated spatial structure of the Voronoi graph, they work with the complete graph on $Q$, where every point is adjacent to each other. They fix a parameter $\alpha>1$, and define the passage time $t_{b}$ of the bond $b$ connecting the lattice points $q$ and $q^{\prime}$ to be

$$
t_{b}=\left|q-q^{\prime}\right|^{\alpha}
$$

where $|\cdot|$ denotes the Euclidean norm in $\mathbb{R}^{d}$. By the triangle inequality of the norm, if $0 \leq \alpha \leq 1$ then the passage time between $q$ and $q^{\prime}$ is trivially minimized by taking the single edge between them. However, when $\alpha>1$, long jumps are discouraged and the model is non-trivial.

Howard and Newman [HN97] prove a shape theorem with limiting shape a Euclidean ball. Consequently, many of the results presented for Standard FPP in Section 1.1 under restrictive hypotheses hold automatically for Euclidean FPP in for all $\alpha>1$. In [HN00], Howard and Newman prove the shape fluctuation exponent bound $\chi \leq 1 / 2$ using a moderate-deviations estimate similar to Kesten's [Kes93], as well as the inequality (1.7) so that $\xi \leq 3 / 4$. Howard [How00] proves the inequality (1.8) and the lower bound $\xi \geq 1 /(d+1)$.

For geodesics, Howard and Newman [HN00] show that with probability one, every one-sided geodesic has an asymptotic direction, and for every $w \in S^{d-1}$ there exists a one-sided geodesic with asymptotic direction $w$. In the $\alpha \geq 2$, they have slightly stronger results [HN97], which they believe should also hold for the $\alpha>1$ case. As with other models of FPP, the existence of two-sided geodesics is still open. The strongest theorem on two-sided geodesics in this setting [HN01] is as with Standard FPP: with probability one, all two-sided geodesics (if they exist) must have antipodal asymptotic directions; and that for any deterministic $w \in S^{d-1}$, with probability one 
there do not exist any two-sided geodesics with asymptotic directions $\pm w$.

\subsubsection{Last-Passage Percolation}

Consider the two-dimensional lattice restricted to the upper-right quadrant,

$$
\mathbb{Z}_{+}^{2}=\left\{\left(z^{1}, z^{2}\right) \in \mathbb{Z}^{2}: z^{1} \geq 0 \text { and } z^{2} \geq 0\right\}
$$

We assign i.i.d. non-negative passage times $t_{b}$ to each bond $b$, and define the lastpassage times between $z$ and $z^{\prime}$ in $\mathbb{Z}^{2}$ to be

$$
\tau\left(z, z^{\prime}\right)=\sup _{\gamma} \sum_{b \in \gamma} t_{b}
$$

where the supremum is taken over directed paths $\gamma$ moving to the up or to the right. The last-passage time is superadditive,

$$
\tau\left(z, z^{\prime}\right) \geq \tau(z, w)+\tau\left(w, z^{\prime}\right)
$$

rather than subadditive like in FPP, but a superadditive version of Kingman's ergodic theorem [Mar00] can be applied to prove the existence of a time constant, and similarly a shape theorem [Mar04].

Directed FPP models are similar to directed polymer growth in physics, where the role of passage times is replaced by random potentials; we explore this connection further in the next section. The first-passage time between two points represents the minimal energy of a polymer, and the last-passage time the maximal energy.

Even though the models of FPP and LPP are qualitatively different, they are both believed to lie in the KPZ universality class [HM07] and consequently satisfy the KPZ equation (1.6). Most impressively, many exact results (scaling laws, asymptotic distributions, have been found for LPP, mostly for exponentially or geometrically distributed passage times [HM07]. In the case of geometric passage times, Johansson [Joh00] exploits a beautiful connection to random matrix theory by means of 
increasing subsequences of random permutations [BDJ99]. Consider the last-passage time $a_{n}=\tau\left(0, n \mathrm{e}_{1}\right)$. Johansson explicitly computes the time constant $\mu$ for which $\frac{1}{n} a_{n} \rightarrow \mu$ and its variance $\sigma^{2} n^{2 / 3}$, implying that the shape fluctuation exponent $\chi$ is exactly equal to $1 / 3$. More incredibly, he shows that $a_{n}$ satisfies a central limit theorem, and that the normalized random variable

$$
\frac{a_{n}-\mu n}{\sigma n^{1 / 3}}
$$

converges in distribution to the Tracy-Widom distribution [TW94].

\subsubsection{Directed Polymers in a Random Environment}

We describe a model of directed polymer growth in the presence of random impurities, following the survey By Comets, Shiga and Yoshida [CSY04]. This is a particular model of random walk in a random environment which in spirit has many similarities to first-passage percolation. In particular, in a certain temperature regime, the shape and transversal fluctuations of polymers are believed to satisfy the KPZ equation (1.6). The literature on random polymer models is vast; we recommend the books by Giacomin [Gia07] and den Hollander [dH09, Chapter 12].

Fix $d \geq 1$, and consider the state space $\mathbb{N} \times \mathbb{Z}^{d}$. A polymer is a randon path $\left\{\left(j, \omega_{j}\right)\right\}_{j=1}^{n}$ in this space, increasing deterministically in the time coordinate $j$. In the absence of impurities, the distribution of the path $\omega_{j}$ will a simple random walk in $\mathbb{Z}^{d}$ starting from the origin. The effects of the impurities are summarized by random variables $\eta(n, x)$ at each site of $\mathbb{N} \times \mathbb{Z}^{d}$. These variables $\eta$ represent random potential energies, and polymers will tend toward sites where $\eta$ is positive. We scale the energies by the non-negative parameter $\beta$. As we will see, there is a phase transition in $\beta$, which depends on the dimension $d$.

We present the model formally. Let $\left(\Omega_{0}, \mathcal{F}_{0}, \mathbb{P}\right)$ be a probability space, and let $\eta=\{\eta(n, x)\}$ be a family of i.i.d. random variables on $\Omega_{0}$ indexed by $\mathbb{N} \times \mathbb{Z}^{d}$. The value $\eta(n, x)$ represents the potential energy at time $n \in \mathbb{N}$ at site $x \in \mathbb{Z}^{d}$. Write 
$\mathbb{E}$ for expectation with respect to $\mathbb{P}$, and suppose that $\eta(n, x)$ has a finite momentgenerating function:

$$
\mathbb{E}\left[\mathrm{e}^{\beta \eta(n, x)}\right]<\infty
$$

for all $\beta \in \mathbb{R}$.

Write $\Omega$ for the space of paths $\omega=\left\{\omega_{j}\right\}$ in $\mathbb{Z}^{d}$ starting at the origin. For $n>0$, define the random Hamiltonians $H_{n}: \Omega \rightarrow \mathbb{R}$ by

$$
H_{n}(\omega)=-\sum_{j=1}^{n} \eta\left(j, \omega_{j}\right)
$$

Let $\mathcal{F}$ be the cylinder $\sigma$-algebra on $\Omega$, and let $\nu$ be the probability measure on $(\Omega, \mathcal{F})$ so that under $\nu, \omega \in \Omega$ is a simple random walk on $\mathbb{Z}^{d}$ starting from the origin.

The measure $\nu$ is a background measure on the space of paths $\Omega$, which we modify by the effect of the environment. Let $\beta \geq 0$ be a non-negative parameter, and define the random Gibbs measure $\mu_{n}$ on $\Omega$ by

$$
\mu_{n}(\omega)=Z_{n}^{-1} \mathrm{e}^{-\beta H_{n}(\omega)} \mathrm{d} \nu(\omega)
$$

where the normalizing constant $Z_{n}=\int_{\Omega} \mathrm{e}^{-\beta H_{n}(\omega)} \mathrm{d} \nu(\omega)$ is called the partition function of $\mu_{n}$. An important quantity is the free energy

$$
F_{n}=-\frac{1}{\beta} \log Z_{n}
$$

The Gibbs measure $\mu_{n}$, the partition function $Z_{n}$ and the free energy $F_{n}$ are all random with respect to the probability measure $\mathbb{P}$.

The parameter $\beta$ represents the inverse temperature (precisely, $\beta^{-1}$ equals the temperature multiplied by the Boltzmann constant). When $\beta=0$, the system is in the infinite-temperature regime, and polymers are exactly simple random walks. When $\beta \approx 0$ (high temperature), if polymers behave similarly to simple random walk, we say the system is in the weak-disorder phase. Conversely, when $\beta \rightarrow \infty$, the thermal fluctuations of polymers is suppressed, and they should behave like minimizing 
paths in first-passage percolation. We call this the strong-disorder phase. Things are qualitatively quite different in the two regimes: as we have seen in the previous sections, minimizing paths in FPP are superdiffusive, whereas simple random walk is diffusive.

The phase transition depends on the dimension $d$ : if $d=1$ or 2 , the system is in the strong disorder phase in the presence of any disorder (i.e. $\beta \neq 0$ ); in the case $d \geq 3$, there exists a non-trivial critical value $\beta_{c}>0$ such that when $\beta<\beta_{c}$ the system is in the weak disorder phase, and when $\beta>\beta_{c}$ it is in the strong disorder phase [CSY04]. Derrida [Der90] has estimates on the critical value $\beta_{c}$ in terms of the dimension $d$.

The model was introduced in the physics literature by Huse and Henley [HH85] in order to model interface boundaries in the low-temperature regime of an Ising model with random impurities. In the $d=1$, strong disorder phase (which corresponds to 2-dimensional FPP), they gave numerical evidence that the transversal fluctuations of polymers should scale like $n^{\xi}$ for $\xi=2 / 3$. Soon after, this value for $\xi$ was confirmed by Huse, Henley and Fisher [HHF85] and Kardar and Nelson [KN85] using heuristic, physical arguments.

In Section 1.1.3, we saw that the shape fluctuation exponent $\chi$ is related to the variance of the passage time $a_{n}=\tau\left(0, n \mathrm{e}_{1}\right)$ in Standard FPP. Here, the role of passage times is replaced by energy, and $\chi$ is related to the fluctuations of the free energy $F_{n}$ :

$$
\operatorname{Var} F_{n} \sim n^{2 \chi}
$$

where the variance is with respect to the probability measure $\mathbb{P}$. It is strongly believed [KS91] that this model also lies in the KPZ universality class, hence satisfies the KPZ equation

$$
\chi=2 \xi-1
$$

in all dimensions $d$. When $d=1$, it is believed that $\chi=1 / 3$, as with two-dimensional Standard FPP. 
Imbrie and Spencer [IS88] formulated the mathematical model described above; for the Bernoulli potential $\eta= \pm 1$, they rigorously showed the phase transition in $d \geq 3$ using an expansion in the small parameter $\beta$. Bolthausen [Bol89] reproved their result using a simple martingale method, which Song and Zhou [SZ96] extended for general environments $\eta$. Adapting the uniform-curvature assumption for Standard FPP as described in Sections 1.1.3 and 1.1.4, Piza [Piz97] proved many rigorous results on fluctuation exponents in this model.

When $d \geq 3$ and $\beta$ is small (weak-disorder phase), one expects polymers to behave roughly like simple random walks. Carmona and $\mathrm{Hu}$ [CH02] proved a theorem on delocalization of polymers in a Gaussian random potential $\eta$ (later improved to general potentials by Comets, Shiga and Yoshida [CSY03]). Recall that a simple random walk $\omega_{n}$ is typically a distance $O(\sqrt{n})$ from the origin. There are $O\left(n^{d / 2}\right)$ points near the surface of the $d$-sphere of radius $\sqrt{n}$, and the probability that $\omega_{n}$ lies at any particular one is $O\left(n^{-d / 2}\right)$ :

$$
\max _{z \in \mathbb{Z}^{d}} \nu\left(\omega_{n}=z\right)=O\left(n^{-d / 2}\right)
$$

where $\nu$ is the simple random walk measure on the space of paths $\Omega$. This is the $\beta=0$ case for random polymers; the precise statement for $d \geq 3$ and small $\beta$ is that

$$
\lim _{n \rightarrow \infty} \max _{z \in \mathbb{Z}^{d}} \mu_{n-1}\left(\omega_{n}=z\right)=0
$$

for $\mathbb{P}$-almost every environment $\eta$. In the strong disorder phase the situation is very different, and polymers are strongly localized. The same authors proved [CH02, CSY03] that if $d=1$ or 2 and $\beta \neq 0$, or if $d \geq 3$ and $\beta$ is large enough, then there exists non-random $c>0$ such that

$$
\limsup _{n \rightarrow \infty} \max _{z \in \mathbb{Z}^{d}} \mu_{n-1}\left(\omega_{n}=z\right) \geq c
$$

for $\mathbb{P}$-almost every environment $\eta$. Giacomin and Toninelli [GT06] have more recent results on the nature of the phase transition between delocalization and localization. 


\subsubsection{Spin Glasses}

Spin-glasses are models of interacting particles on a lattice, governed by a Hamiltonian of the form $H(\sigma)=-\sum J_{i j} \sigma_{i} \sigma_{j}$. Unlike a disordered ferromagnet, where the coupling constants $J_{i j}$ are assumed to be random but positive (so that like-spins attract), for a spin-glass model one assumes that the coupling constants $J_{i j}$ can take positive or negative values. This introduces magnetic frustration (nearby spins need not align), which makes the model difficult to study.

Edwards and Anderson [EA75] introduced a particularly simple spin-glass model to describe. Consider a large box $\Lambda \subseteq \mathbb{Z}^{d}$ of size $|\Lambda|=N^{d}$, and the space $\Sigma=$ $\{-1,+1\}^{\Lambda}$ of up-down configurations $\sigma=\left\{\sigma_{i}\right\}$ on $\Lambda$. Let $J_{i j}$ be an i.i.d. family of random variables on a probability space $(\Omega, \mathcal{F}, \mathbb{P})$, and consider the random Hamiltonian $H: \Sigma \rightarrow \mathbb{R}$

$$
H_{N}(\sigma)=-\sum_{|i-j|=1} J_{i j} \sigma_{i} \sigma_{j},
$$

where the sum is over nearest neighbors of $\Lambda$. For $\beta \geq 0$, consider the random Gibbs measure

$$
\mu_{N}(\sigma)=Z_{N}^{-1} \mathrm{e}^{-\beta H_{N}(\sigma)}
$$

with partition function $Z_{N}=\sum_{\sigma} \mathrm{e}^{-\beta H_{N}(\sigma)}$. If the model is ferromagnetic (nonnegative coupling constants $J_{i j} \geq 0$ ), then there are only two ground states: all sites equal to +1 or to -1 . In the general spin-glass model, the all-up and all-down states are $\mathbb{P}$-almost surely no longer ground states. It is an open question if spin-glass models have any non-constant ground states. As mentioned in Section 1.1.5, the twodimensional disordered ferromagnet $\left(J_{i j} \geq 0\right)$ is the dual model to Standard FPP, where the interface boundaries of non-trivial ground states [New97] are the two-sided geodesics on the dual lattice. Consequently,

The spatial structure makes the Edwards-Anderson spin glass extremely difficult to work with. A drastic simplification is to consider a mean-field model, where the 
underlying graph is the complete graph $\Lambda$ on $N$ vertices, and every node on the graph interacts with every other one. Mean-field models are often easier to work with than ones with finite-dimensional interactions.

The Sherrington-Kirkpatrick spin-glass [SK75] is one famous example of a meanfield spin-glass model which has exact solutions. Here the random coupling constants $J_{i j}$ are i.i.d., symmetric random variables with mean zero and variance $J^{2} \gg 1$ (when the variance is small, the system is in a weak-disorder phase hence easier to study). The random Hamiltonian is

$$
H_{N}(\sigma)=-\frac{1}{\sqrt{N}} \sum_{i, j \in \Lambda} J_{i j} \sigma_{i} \sigma_{j},
$$

and the random Gibbs measure

$$
\mu_{N}(\sigma)=Z_{N}^{-1} \mathrm{e}^{-H_{N}(\sigma)}
$$

with partition function $Z_{N}=\Sigma_{\sigma} \mathrm{e}^{-H_{N}(\sigma)}$. The random free energy is

$$
F_{N}=-\log Z_{N}
$$

Using the non-rigorous technique of replica symmetry breaking, Parisi [Par79] calculated an exact form for the free energy in the infinite-volume limit. Aizenman, Lebowitz and Ruelle [ALR87] rigorously calculated the average value of the free energy per site, as well as the fluctuations. Talagrand [Tal98] used his powerful concentration-of-measure techniques to rigorously verify Parisi's full ansatz, and Guerra and Toninelli [GT02] have pushed these techniques further.

As discussed in Section 1.1.5, it is believed that there are no two-sided minimizing geodesics in two-dimensional Standard FPP. When interpreted in the context of the disordered ferromagnet, this means that there are no non-trivial ground states, only the unique ground state. However, this is not believed to be preserved under small perturbations of the metric (a phenomenon called disorder chaos). It is believed that spin-glass models demonstrate the multiple valleys phenomenon: there 
are many very different states which are almost ground states. Chatterjee [Cha08] has shown that chaos and multiple valleys often occur in tandem in general, along with a phenomenon called "superconcentration," when the variance of the free energy is sublinear. In [Cha09] Chatterjee proves that the Sherrington-Kirkpatrick model exhibits superconcentration, chaos and multiple valleys.

Different from the replica method, Mezard, Parisi and Virasoro [MPV87] introduced the ultrametricity assumption to calculate the free energy; see [PRT00] and [ASS07] for more details. Derrida [Der85] developed the Random Energy Model (REM) to formulate a general proof, which Ruelle [Rue87] improved to the probability cascade technique. Arguin and Aizenman [AA09] have recently developed a theory based on multiple valleys to confirm the ultrametricity assumption.

Superconcentration is reminiscent of the sublinear passage-time variation of Benjamini, Kalai and Schramm [BKS03] for Standard FPP discussed in Section 1.1.4, though Chatterjee's demonstration of the phenomenon in the Sherrington-Kirkpatrick model uses very different techniques. The equivalence of the three phenomena has not yet been shown for Standard FPP.

\subsection{Included Papers}

As part of my dissertation work, my advisor Jan Wehr and I wrote the article [LW10], included as Appendix A. It will be published in the May 2010 issue of the Journal of Mathematical Physics. This was a collaborative effort between Prof. Wehr and myself. We wrote this article in order to introduce our continuum model of Riemannian FPP, and demonstrate that we could adapt the basic techniques of Standard FPP for our setting. The main result is a shape theorem: large balls in the Riemannian metric grow roughly like Euclidean ones. As a consequence, we show that the random metric is geodesically complete with probability one.

I wrote the article [LaG10], included as Appendix B, in order to deal with a con- 
ditional probability estimate stemming from the project on geodesics described in Chapter 2. A disintegration (or regular conditional probability) is a way to condition a probability measure on a single point $y$. In that paper, we introduce continuous disintegrations as those which vary continuously in $y$. I present a necessary and sufficient condition for continuous disintegrations to exist for Gaussian measures on separable Banach spaces, and analyze how they transform under absolutely-continuous changes of measure. This project was motivated by the application to Riemannian FPP detailed in Chapter 4; however, the full study of continuous disintegrations was interesting and general enough to warrant submission as a separate publication. 
Chapter 2

Present Study 


\subsection{Geometry Background and Notation}

Before introducing any probabilistic structure, we introduce some geometric notation. Consider $\mathbb{R}^{d}$ with $d \geq 2$ and the standard Euclidean coordinates, and fix $\alpha>0$. Write

$$
\mathrm{SPD}=\{\text { symmetric, positive-definite } d \times d \text { real matrices }\}
$$

and let $g \in C^{2+\alpha}\left(\mathbb{R}^{d}\right.$, SPD) be a $C^{2+\alpha}$-smooth function on $\mathbb{R}^{d}$ with values in the space of symmetric, positive-definite matrices. $g$ defines a Riemannian structure on $\mathbb{R}^{d}$ : for tangent vectors $v, v^{\prime} \in T_{x} \mathbb{R}^{d}$, we consider the inner product $\left\langle v, g(x) v^{\prime}\right\rangle$. For a single tangent vector $v$, we denote by $\|v\|=\sqrt{\langle v, g(x) v\rangle}$ and $|v|=\sqrt{\langle v, v\rangle}$ the Riemannian and Euclidean lengths of $v$, respectively. For a $C^{1}$-curve $\gamma:[a, b] \rightarrow \mathbb{R}^{d}$, we define the Riemannian and Euclidean lengths of $\gamma$ by

$$
R(\gamma)=\int_{a}^{b}\|\dot{\gamma}(t)\| \mathrm{d} t \quad \text { and } \quad L(\gamma)=\int_{a}^{b}|\dot{\gamma}(t)| \mathrm{d} t
$$

respectively. We say that a curve is finite if it has finite Euclidean length; for our model, Theorem 2.2 will imply that finite curves have finite Riemannian length. The Riemannian distance between two points $x$ and $y$ is defined by

$$
d(x, y)=\inf _{\gamma} R(\gamma)
$$

where the infimum is over all $C^{1}$-curves $\gamma$ connecting $x$ to $y$.

A $C^{2}$-curve $\gamma$ is called a geodesic if it locally minimizes the Riemannian energy functional $R^{2}(\gamma)=\frac{1}{2} \int\|\dot{\gamma}\|^{2}$. Equivalently, geodesics are the solutions to the EulerLagrange equation for $R^{2}$,

$$
\ddot{\gamma}^{k}=-\Gamma_{i j}^{k} \dot{\gamma}^{i} \dot{\gamma}^{j}
$$

where we follow the Einstein convention of summing over the repeated indices $i$ and $j$, and where $\Gamma_{i j}^{k}$ are the Christoffel symbols [Lee97] for the metric $g_{i j}$. As this is a second-order system of ordinary differential equations, a geodesic is uniquely determined by its starting point and velocity. Geodesics are locally length-minimizing 
[Lee97]. We call a geodesic $\gamma$ minimizing (or globally minimizing) if for all $x, y \in \gamma$, the Riemannian distance $d(x, y)$ is realized as the Riemannian length of $\gamma$ from $x$ to $y$. Not all geodesics are minimizing; for example, on the sphere, the geodesics are great circles, which are not minimizing past antipodal points. Geodesics have constant speed [Lee97]; henceforth, we assume $\|\dot{\gamma}\|=1$ so that geodesics are parametrized by Riemannian arc length.

For a Riemannian metric $g$, we define the real, positive functions

$\Lambda(x)=$ maximum eigenvalue of $g(x) \quad$ and $\quad \lambda(x)=$ minimum eigenvalue of $g(x)$.

For any $K \subseteq \mathbb{R}^{d}$, define

$$
\Lambda(K)=\sup _{x \in K} \Lambda(x) \quad \text { and } \quad \lambda(K)=\inf _{x \in K} \lambda(x) .
$$

By the continuity and positivity of $g$, if $K$ is bounded then

$$
0<\lambda(K) \leq \Lambda(K)<\infty
$$

For $z \in \mathbb{Z}^{d}$, let $C_{z}=[z-1 / 2, z+1 / 2)^{d}$ be the unit cube centered at $z$. Write

$$
\Lambda_{z}=\Lambda\left(C_{z}\right) \quad \text { and } \quad \lambda_{z}=\lambda\left(C_{z}\right)
$$

\subsection{Riemannian FPP}

We consider the probability space $\Omega=C^{2+\alpha}\left(\mathbb{R}^{d}, \mathrm{SPD}\right)$ with the $\sigma$-algebra $\mathcal{F}$ generated by cylinder sets. This space $\Omega$ is a topological subspace of the Fréchet space $\hat{\Omega}=$ $C^{2+\alpha}\left(\mathbb{R}^{d}\right.$, Sym $)$, where Sym is the space of symmetric $d \times d$ real matrices with matrix norm.

We call $\Omega$ the space of Riemannian metrics on $\mathbb{R}^{d}$. Let $g$ be an $\Omega$-valued random variable with a Radon probability distribution $\mathbb{P}$ satisfying the following four assumptions:

\section{Assumption 2.1.}


a. $\mathbb{P}$ is isotropic, that is, invariant under the isometries of $\mathbb{R}^{d}$, rotations, translations and reflections.

b. $\mathbb{P}$ has finite-range dependence. i.e., there exists $\xi>0$ such that if $|x-y| \geq \xi$, then $g(x)$ and $g(y)$ are independent.

c. The random variables $\Lambda_{0}$ and $\Lambda_{0} / \lambda_{0}$ have finite moment-generating functions. That is,

$$
\mathbb{E}\left[\mathrm{e}^{r \Lambda_{0}}\right]<\infty \quad \text { and } \quad \mathbb{E}\left[\mathrm{e}^{r \Lambda_{0} / \lambda_{0}}\right]<\infty
$$

for some $r>0$. Since $\lambda_{0} \leq \Lambda_{0}$, it follows that $\mathbb{E}\left[\mathrm{e}^{r \lambda_{0}}\right]<\infty$.

d. There exists a stationary, mean-zero Gaussian measure $\hat{\mathbb{P}}$ on $\hat{\Omega}$ such that $\mathbb{P}$ is absolutely continuous with respect to $\hat{\mathbb{P}}$ on $\hat{\Omega}$; and the Radon-Nikodym derivative $\frac{d \mathbb{P}}{d \hat{\mathbb{P}}}(g)$ is bounded and continuous, and is positive exactly on the open set $\Omega \subseteq \hat{\Omega}$.

The first three assumptions imply that $(\Omega, \mathcal{F}, \mathbb{P})$ satisfies the hypotheses of [LW10], including a shape theorem with limiting shape equal to a Euclidean ball and almostsure completeness of the metric $g$. We summarize these results in this theorem:

\section{Theorem 2.2.}

a) There exists $\mu>0$ such that $\frac{1}{t} d(0, t v) \rightarrow \mu$ a.s. and in $L^{1}$, uniformly in the direction $v \in S^{d-1}$. Precisely, for all $\epsilon>0$, with probability one, there exists $T>0$ such that if $t \geq T$, then $|d(0, t v)-\mu t| \leq \epsilon t$ for any $v \in S^{d-1}$.

b) Let $A=\left\{x:|x| \leq \mu^{-1}\right\}$ and $B_{t}=\{x: d(0, x) \leq t\}$ be the Euclidean and Riemannian balls centered at the origin of radius $\mu^{-1}$ and $t$, respectively. For all $\epsilon>0$, with probability one, there exists $T>0$ such that if $t \geq T$, then

$$
(1-\epsilon) A \subseteq \frac{1}{t} B_{t} \subseteq(1+\epsilon) A
$$

The Euclidean ball $A$ is called the limiting shape of the model. 
c) With probability one, the Riemannian metric $g$ is geodesically complete. Consequently, with probability one, for all $x$ and $y$ in $\mathbb{R}^{d}$, there is a finite, minimizing geodesic $\gamma$ connecting $x$ to $y$ such that $d(x, y)=R(\gamma)$.

Proof. The constant $\mu$ is independent of the direction $v$ since the measure $\mathbb{P}$ is rotationally-invariant. Part (a) is Proposition 3.3 of [LW10]. Part (b) is Theorem 3.1 of [LW10]. Part (c) is Corollary 3.5 of [LW10].

\subsection{The evolution of the environment under the geodesic flow}

For each $x \in \mathbb{R}^{d}$, the matrix $g(x)$ is positive-definite, hence invertible. Omitting the $x$, we write $g$ in coordinates as $g_{i j}$, and its inverse $g^{-1}$ as $g^{i j}$. We define the Christoffel symbols [Lee97]

$$
\Gamma_{i j}^{k}=\frac{1}{2} g^{k m}\left(\frac{\partial}{\partial x^{i}} g_{m j}+\frac{\partial}{\partial x^{j}} g_{i m}-\frac{\partial}{\partial x^{m}} g_{i j}\right)
$$

where we follow the Einstein notation by summing over repeated indices. Geodesics are the solutions to the equation

$$
\ddot{\gamma}^{k}=-\Gamma_{i j}^{k} \dot{\gamma}^{i} \dot{\gamma}^{j}
$$

In terms of a vector field $U: \mathbb{R}^{2 d} \rightarrow \mathbb{R}^{2 d}$, geodesics are the flow lines for

$$
U(x, v)=\left(v,-\Gamma_{i j}^{k}(x) v^{i} v^{j} \mathrm{e}_{k}\right)
$$

where $\mathrm{e}_{k}$ is the $k^{\text {th }}$ standard basis vector in $\mathbb{R}^{d}$. Let $F_{t}: \mathbb{R} \times \mathbb{R}^{2 d} \rightarrow \mathbb{R}^{2 d}$ be the geodesic flow, so that $\frac{\mathrm{d}}{\mathrm{d} t} F_{t}=U\left(F_{t}\right)$ and

$$
F_{t}(x, v)=\left(\gamma_{x, v}(t), \dot{\gamma}_{x, v}(t)\right)
$$

where $\gamma_{x, v}$ is the unique geodesic starting at $x$ in the direction $v$. By assumption, the

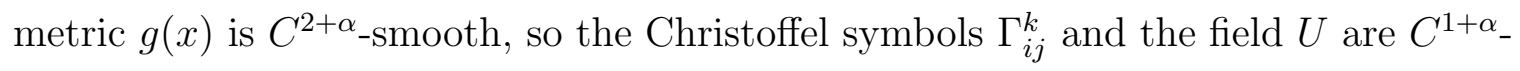
smooth. Consequently, the flow $F_{t}$ is $C^{1+\alpha}$-smooth [AL88] hence locally Lipschitz. 
Fix $v \in S^{d-1}$. Rather than fixing the environment $g \in \Omega$ and considering the flow $F_{t}(0, v)$ along the geodesic $\gamma_{v}:=\gamma_{0, v}$ (the Eulerian perspective), we instead consider a reference frame centered along a particle traveling along $\gamma_{v}$ (the Lagrangian perspective). Define the random flow $\sigma_{t}: \Omega \rightarrow \Omega$ on the space of Riemannian metrics by

$$
\left(\sigma_{t} g\right)(u)=g\left(u+\gamma_{v}(t)\right)
$$

The variable $u \in \mathbb{R}^{d}$ represents the displacement from $\gamma_{v}(t)$, so that $\left(\sigma_{t} g\right)(0)=$ $g\left(\gamma_{v}(t)\right)$ always represents the metric at $\gamma_{v}(t)$. The flow $\sigma_{t}$ induces the random measure $\mathbb{P} \circ \sigma_{t}^{-1}$ on $\Omega$.

Claim 2.3. Fix $v \in S^{d-1}$. With probability one, for all $t \in \mathbb{R}$ the random measure $\mathbb{P} \circ \sigma_{t}^{-1}$ on $\Omega$ is absolutely continuous with respect to $\mathbb{P}$. That is, there exists a family of measurable functions $\rho_{t}: \Omega \rightarrow \mathbb{R}$ so that for all measurable $f: \Omega \rightarrow \mathbb{R}$,

$$
\int_{\Omega} f\left(\sigma_{t} g\right) \mathrm{d} \mathbb{P}(g)=\int_{\Omega} f(g) \rho_{t}(g) \mathrm{d} \mathbb{P}(g) .
$$

This should follow from the main theorem of Geman and Horowitz [GH75]; see Zirbel [Zir01] for a more recent presentation. They call a vector field homogeneous if its law is translation-invariant. By the isotropy of $\mathbb{P}$, the vector field $U(x, v)$ is homogeneous in the first coordinate. If $U$ were homogeneous in both coordinates, then the claim would immediately follow by Proposition 8.2 of [Zir01]. However, this is not the case, and their work must be modified for this situation.

\subsection{Rarity of Minimizing Geodesics}

Consider the set of all minimizing, unit-speed geodesics between the origin and the boundary sphere of the Euclidean ball $B_{n}^{\mathrm{E}}:=B^{\mathrm{E}}(0, n)$ of radius $n$. Let $\mathcal{V}_{n} \subseteq S^{d-1}$ be the set of initial velocities of these geodesics. Note that these geodesics may exit $B_{n}^{\mathrm{E}}$. Clearly, $\mathcal{V}_{n}$ is monotonically decreasing in the sense that $\mathcal{V}_{n+1} \subseteq \mathcal{V}_{n}$. Let $\mathcal{V}=\bigcap \mathcal{V}_{n}$ 
Proposition 2.4. The set $\mathcal{V}$ is non-empty and closed.

Proof. Since the metric is complete with probability one, each distance $d\left(0, n \mathrm{e}_{1}\right)$ is realized by a finite minimizing geodesic $\gamma_{n}$ connecting 0 to $n \mathrm{e}_{1}$. Let $v_{n} \in S^{d-1}$ be the initial velocities of these geodesics. Since the sphere is compact, a subsequence $v_{n_{k}}$ converges to some $v \in S^{d-1}$. Let $\gamma$ be the unique geodesic with $\gamma(0)=0$ and $\dot{\gamma}(0)=v$, parametrized by Riemannian length. We claim that $\gamma$ is minimizing.

Let $x=\gamma(t)$ and $x^{\prime}=\gamma\left(t^{\prime}\right)$ be two points along the curve $\gamma$. As mentioned in the previous section, the geodesic flow is smooth in the initial conditions, so

$$
x=\lim _{k \rightarrow \infty} \gamma_{n_{k}}(t) \quad \text { and } \quad x^{\prime}=\lim _{k \rightarrow \infty} \gamma_{n_{k}}\left(t^{\prime}\right)
$$

Since the distance function $d$ is continuous and the finite geodesics $\gamma_{n_{k}}$ are minimizing,

$$
d\left(x, x^{\prime}\right)=\lim _{k \rightarrow \infty} d\left(\gamma_{n_{k}}(t), \gamma_{n_{k}}\left(t^{\prime}\right)\right)=\left|t-t^{\prime}\right|
$$

This proves that $\gamma$ globally minimizes length.

In fact, the same argument shows that $\mathcal{V}$ is closed. Let $v_{n} \in \mathcal{V}$, and suppose that $v_{n} \rightarrow v$ in $S^{d-1}$. Let $\gamma_{n}$ and $\gamma$ be the geodesics starting at the origin in directions $v_{n}$ and $v$, respectively. The above argument shows that $\gamma$ is minimizing, so $v \in \mathcal{V}$.

We call $w$ an asymptotic direction of $\gamma$ if the limit of $\gamma(t) /|\gamma(t)|$ exists and equals $w$ as $t \rightarrow \infty$. Howard and Newman [HN97] have shown that for their rotationallyinvariant model of Euclidean first-passage percolation, every one-sided minimizing geodesic has an asymptotic direction. The key to their proof is that the limiting shape of Euclidean FPP is a Euclidean ball. Since the global structure of both their model and our own are similar, we conjecture that the same is true in this setting:

Conjecture 2.5. For every $v \in \mathcal{V}$, there exists $w \in S^{d-1}$ such that

$$
\lim _{t \rightarrow \infty} \frac{\gamma_{v}(t)}{\left|\gamma_{v}(t)\right|}=w
$$


If this conjecture holds, then we should be able to improve non-emptyness of $\mathcal{V}$ to uncountability:

Corollary 2.6. If Conjecture 2.5 holds, then the set $\mathcal{V}$ is uncountable.

We claim that for each $w \in S^{d-1}$, there is some $v \in \mathcal{V}$ so that $\gamma_{v}$ has asymptotic direction $w$. The proof mimics that of Proposition 2.4: we begin with the minimizing geodesics $\gamma_{n}$ from 0 to $n w$, and take the limit of a subsequence of their starting directions $\dot{\gamma}_{n_{k}}(0) \rightarrow v$. By Conjecture 2.5, $\gamma_{v}$ has an asymptotic direction $w^{\prime}$. An additional argument is needed to show that $w=w^{\prime}$; for example a result that the transversal fluctuation exponent $\xi$ is less than 1, as for lattice FPP models [How04].

The main goal of this project is to show that for a deterministic $v \in S^{d-1}$, the geodesic $\gamma_{v}$ starting in direction $v$ is length minimizing with probability zero.

Claim 2.7. For each $v \in S^{d-1}$, the event

$$
\{v \in \mathcal{V}\}=\left\{\gamma_{v} \text { is minimizing }\right\}
$$

has probability zero.

This is a new result for this model for which there is no analogue in lattice FPP models. At the time of submitting this dissertation, we do not yet have a full proof of this claim. However, we have formulated the general argument of the proof, as well as many technical lemmas. We plan to work through all the technical details and submit this soon for publication.

In Section 2.5, we prove that minimizing geodesics are transient, i.e. leave every compact set. We use this along with some results on dependent lattice FPP which we developed in [LW10] to prove a global existence statement: there are a number of "frontier times" along a minimizing geodesic $\gamma_{v}$ at which things are "well-behaved." In Section 2.6, using the continuous disintegrations which we developed for stationary 
Gaussian measures in [LaG10], we show that at each of these times $t_{k}$, there is a uniform probability estimate for a destabilizing phenomenon to occur in front of $\gamma_{v}\left(t_{k}\right)$. In Section 2.7, we argue that this phenomenon should be a "bump surface," to exploit positive curvature so that the curve $\gamma_{v}$ develops conjugate points.

By a standard application of Tonelli's theorem [Fol99], Claim 2.7 can be improved to show that, with probability one, $\mathcal{V}$ has measure zero on the sphere $S^{d-1}$. This is not just a technicality: in light of Corollary 2.6, we believe that $\mathcal{V}$ is uncountable. We further believe that $\mathcal{V}$ has no isolated points and is nowhere dense, so that the set of minimizing directions is a random topological Cantor set.

Theorem 2.8. If Claim 2.7 holds, then with probability one, the set $\mathcal{V}$ has measure zero on the sphere $S^{d-1}$. Precisely, if $\nu$ is the uniform measure on $S^{d-1}$, then

$$
\mathbb{P}(\nu(\mathcal{V})=0)=1
$$

Proof. For $v \in S^{d-1}$, let $E_{v}=\{v \notin \mathcal{V}\}$ be the event that the geodesic $\gamma_{v}$ is not minimizing. Claim 2.7 implies that $\mathbb{P}\left(E_{v}\right)=1$. Write $\mathcal{V}^{c}=\left\{v \in S^{d-1}: E_{v}\right.$ occurs $\}$ for the directions which do not give minimizing geodesics, and let $\nu$ be the uniform measure on $S^{d-1}$. Tonelli's theorem [Fol99] implies that

$$
\begin{aligned}
\int_{\Omega} \nu\left(\mathcal{V}^{c}\right) \mathrm{d} \mathbb{P}(\omega) & =\int_{\Omega} \nu\left(v: E_{v} \text { occurs }\right) \mathrm{d} \mathbb{P}(\omega)=\int_{\Omega} \int_{S^{d-1}} 1_{E_{v}}(\omega) \mathrm{d} \nu(v) \mathrm{d} \mathbb{P}(\omega) \\
& =\int_{S^{d-1}} \int_{\Omega} 1_{E_{v}}(\omega) \mathrm{d} \mathbb{P}(\omega) \mathrm{d} \nu(v)=\int_{S^{d-1}} \mathbb{P}\left(E_{v}\right) \mathrm{d} \nu(v)=\int_{S^{d-1}} 1 \mathrm{~d} \nu(v)=1,
\end{aligned}
$$

since $\mathbb{P}\left(E_{v}\right)=1$. Thus $\nu\left(\mathcal{V}^{c}\right)=1$ with probability one, so $\nu(\mathcal{V})=0$.

\subsection{Transience of Geodesics and Existence of Frontier Times}

As part of their definition in Section 2.1, geodesics are parametrized by Riemannian arc length, so $\|\dot{\gamma}(t)\|=1$ for all $t$. This is the natural parametrization from the point of view of differential geometry, as it depends only on the intrinsic geometry. In our probabilistic model, the initial Euclidean coordinate system is also natural. Since 
geodesics are curves in $\mathbb{R}^{d}$, we will also consider them parametrized by Euclidean arc length $l$, so that $|\dot{\gamma}(l)|=1$ for all $l$.

The following theorem demonstrates that minimizing geodesics are transient, whether parametrized by Riemannian or Euclidean length. We show that for any (possibly random) compact set $K$, there exists a uniform time after which all minimizing geodesics never return to $K$. We use the notation $\gamma_{v}$ to mean the unique geodesic starting at 0 in direction $v \in S^{d-1}$.

\section{Theorem 2.9.}

a) Suppose that geodesics are parametrized by Riemannian arc length $t$. With probability one, if $K$ is a (possibly random) compact set in $\mathbb{R}^{d}$, then there exists a time $T$ such that for all $v \in \mathcal{V}$ and $t>T, \gamma_{v}(t) \notin K$.

b) Suppose that geodesics are parametrized by Euclidean arc length $l$. With probability one, if $K$ is a (possibly random) compact set in $\mathbb{R}^{d}$, then there exists a time $L$ such that for all $v \in \mathcal{V}$ and $l>L, \gamma_{v}(l) \notin K$.

We require almost-sure completeness of the metric in our proof of part (b), where we assume that a Riemannian ball of finite radius must be compact in $\mathbb{R}^{d}$.

Proof of a). Let $\hat{K}=B^{\mathrm{E}}(0, r)$ be the smallest Euclidean ball centered at the origin which contains $K$. The metric $g$ is continuous hence bounded on the ball $\hat{K}$, so the maximum eigenvalue $\Lambda(\hat{K})$ is finite. Let $T=r \sqrt{\Lambda(\hat{K})}$.

Let $v \in \mathcal{V}$ and suppose that $\gamma_{v}$ is the unique geodesic starting at the origin in direction $v$. If $\gamma_{v}(t) \in K$ for some time $t$, then since $\gamma_{v}$ is minimizing,

$$
t=d\left(0, \gamma_{v}(t)\right) \leq r \sqrt{\Lambda(\hat{K})}=T
$$

where we estimate the distance by the Riemannian length of the straight-line path between 0 and $\gamma_{v}(t)$. Thus, if $t>T$, then $\gamma_{v}(t) \notin K$. 
Proof of b). For a (possibly random) compact set $K$, let $T$ be as in part (a). Consider $B=B^{\mathrm{R}}(0, T)$, the closed Riemannian ball centered at the origin of radius $T$. By the almost-sure completeness of the metric, $B$ is compact. The metric is positivedefinite and continuous, so $\lambda(B)>0$. Let $L=T / \sqrt{\lambda(B)}$.

Let $v \in \mathcal{V}$ and suppose that $\gamma_{v}(l) \in K$; we will show that $l \leq L$. Let $t(l)=$ $\int_{0}^{l} \sqrt{\left\langle\dot{\gamma}_{v}, g \dot{\gamma}_{v}\right\rangle}$ be the Riemannian arc length of $\gamma_{v}$ from 0 to $l$. Since $\gamma_{v}(l) \in K$, the above argument shows that $t(l) \leq T$. Furthermore, since $t$ is an increasing function of $l$, for all $l^{\prime} \leq l$ the Riemannian times $t\left(l^{\prime}\right)$ are bounded above by $T$, hence $\gamma_{v}\left(l^{\prime}\right) \in B$. Thus

$$
T \geq t(l)=\int_{0}^{l} \sqrt{\left\langle\dot{\gamma}_{v}, g \dot{\gamma}_{v}\right\rangle} \geq l \sqrt{\lambda(B)},
$$

since $\gamma_{v}$ is parametrized by Euclidean arc length so $\left\langle\dot{\gamma}_{v}, \dot{\gamma}_{v}\right\rangle=1$. Therefore, $l \leq$ $T / \sqrt{\lambda(B)}=L$.

The next theorem is an improvement on the previous one. Not only are minimizing geodesics transient, but for each $v \in \mathcal{V}$, there exists a sequence of "frontier times" $t_{k}(v) \uparrow \infty$ such that things are "nice" at $\gamma_{v}\left(t_{k}\right)$. First, the geodesic satisfies a cone condition at these times: there is a uniform $\theta<\frac{\pi}{2}$ such that the angle between $\gamma_{v}\left(t_{k}\right)$ and $\dot{\gamma}_{v}\left(t_{k}\right)$ is less than $\theta$. In particular, this means that at $t_{k}$, the geodesic is not tangent to the Euclidean ball of radius $\left|\gamma_{v}\left(t_{k}\right)\right|$ centered at the origin. Second, there is a uniform upper bound on the $C^{2+\alpha}$-norm of the metric $g$ in a uniform neighborhood $B_{k}$ of $\gamma_{v}\left(t_{k}\right)$, as well as a lower bound on $\lambda$, the minimum eigenvalue of $g$.

Let $\xi$ be the finite-dependence length of the metric. i.e., if $|u-v| \geq \xi$, then $g(u)$ and $g(v)$ are independent.

Theorem 2.10. There exist non-random $\beta \in(0,1)$ and $h>0$ such that, with probability one, for all $v \in \mathcal{V}$, there exists a sequence of "frontier times" $t_{k}(v) \uparrow \infty$ such that

- The angle between $\gamma_{v}\left(t_{k}\right)$ and $\dot{\gamma}_{v}\left(t_{k}\right)$ is at most $\theta:=\cos ^{-1} \beta$, uniformly in $k$. 
i.e.,

$$
\left\langle\gamma_{v}\left(t_{k}\right), \dot{\gamma}_{v}\left(t_{k}\right)\right\rangle \geq\left|\gamma_{v}\left(t_{k}\right)\right|\left|\dot{\gamma}_{v}\left(t_{k}\right)\right| \cos \theta=\left|\gamma_{v}\left(t_{k}\right)\right|\left|\dot{\gamma}_{v}\left(t_{k}\right)\right| \beta
$$

- Let $\rho=2 \xi / \beta$, for $\xi$ as above. Write

$$
B_{k}=B^{\mathrm{E}}\left(\gamma\left(t_{k}\right), \rho\right)
$$

for the Euclidean ball of radius $\rho$ centered at $\gamma\left(t_{k}\right)$. Then

$$
\|g\|_{C^{2+\alpha}\left(B_{k}\right)}+\frac{1}{\lambda\left(B_{k}\right)} \leq h .
$$

The event in this theorem holds simultaneously for all directions in the set $\mathcal{V}$ with probability one, though the particular sequence of times $t_{k}$ depends on the direction $v$. In fact, we will prove this theorem for arbitrary $\rho$ in (2.2), though in that case the non-random constant $h$ will depend on $\rho$. The proof is technical, and uses some lemmas from [LW10]. It can be found in Chapter 3.

\subsection{Uniform Probability Estimates at Frontiers}

For this section, we fix $v \in S^{d-1}$, and consider the unique geodesic $\gamma_{v}$ starting from the origin in direction $v$. If $\gamma_{v}$ is to be minimizing, a necessary condition will be that there is a sequence of "frontier times" along the geodesic. We argue in Claim 2.14 that at each of these times, there is a uniform probability $p$ with which a certain event occurs.

To this more precise, we consider a filtration, ordered by space rather than time. Since minimizing geodesics are transient, a natural filtration to consider is

$$
\mathcal{F}_{r}:=\sigma\{g(x):|x| \leq r\}
$$

the $\sigma$-algebra generated by the metric in the closed Euclidean ball $B_{r}^{\mathrm{E}}=B^{\mathrm{E}}(0, r)$. 
Define the random function $\tau_{v}:[0, \infty) \rightarrow[0, \infty]$ as the first time that $\gamma_{v}$ leaves the ball of radius $r$. That is,

$$
\tau_{v}(r)=\inf \left\{t:\left|\gamma_{v}(t)\right|=r \text { and is increasing }\right\}
$$

where $\tau_{v}(r)=\infty$ if $\gamma_{v}$ is trapped in the ball $B_{r}^{\mathrm{E}}$ for all time (i.e. $\left|\gamma_{v}(t)\right| \leq r$ for all $t$ ). Where they are finite, the random functions $\tau_{v}(r)$ are all strictly increasing and right-continuous with left limits. The exit times $\tau_{v}(r)$ depend only on the metric in the Euclidean ball of radius $r$, hence are adapted to the filtration $\mathcal{F}_{r}$. For transient geodesics $\gamma_{v}$ (including minimizing geodesics by Proposition 2.9), the exit time $\tau_{v}(r)$ is finite for all $r$.

Let $\beta, h$ and $\rho=2 \xi / \beta$ be as in Theorem 2.10, and fix $v \in S^{d-1}$. We will call $R$ a frontier of $\gamma_{v}$ if the exit time $t:=\tau_{v}(R)$ is finite and satisfies the conclusions of Theorem 2.10, where $B_{k}$ is replaced by

$$
B=B^{\mathrm{E}}(\gamma(t), \rho) \cap B_{R}^{\mathrm{E}}
$$

the part of the neighborhood around $\gamma(t)$ which is contained in the large ball $B_{R}^{\mathrm{E}}=$ $B^{\mathrm{E}}(0, R)$.

Definition 2.11. We define $R \geq 0$ to be a frontier of $\gamma_{v}$ if the exit time $t:=\tau_{v}(R)$ is finite, the angle between $\gamma_{v}\left(t_{k}\right)$ and $\dot{\gamma}_{v}\left(t_{k}\right)$ is at most $\theta:=\cos ^{-1} \beta$, uniformly in $k$, and

$$
\|g\|_{C^{2+\alpha}(B)}+\frac{1}{\lambda(B)} \leq h .
$$

Frontiers are "stopping times" (in the probabilistic sense) with respect to the filtration $\mathcal{F}_{r}$, since the event

$$
\left\{R \text { is a frontier of } \gamma_{v}\right\} \cap\{R \leq r\}
$$

depends only on the metric in the ball $B_{r}^{\mathrm{E}}$ (i.e. the event is $\mathcal{F}_{r}$-measurable). Theorem 2.10 implies that there is a sequence of frontiers along minimizing geodesics: 
Corollary 2.12. With probability one, if $v \in \mathcal{V}$, then there is a sequence of frontiers $R_{k} \uparrow \infty$ along $\gamma_{v}$.

Let $v \in S^{d-1}$. We will use frontiers to test if $v \in \mathcal{V}$. If we can not find a sequence of frontiers $R_{k}$ along $\gamma_{v}$, then Corollary 2.12 implies that $v \notin V$. If there does exist such a sequence $R_{k}$, then our Claim 2.14 will imply that there is a uniform probability $p$ so that at each frontier time $R_{k}$, the geodesic $\gamma_{v}$ encounters a phenomenon which destabilizes the minimization property.

Let $\mathcal{O}_{x}: \mathbb{R}^{d} \rightarrow \mathbb{R}^{d}$ be a family of affine transformations on $\mathbb{R}^{d}$ which map $0 \mapsto x$ and $-|x| \mathrm{e}_{1} \mapsto 0 .{ }^{1}$ Fix $v \in S^{d-1}$, and define the $\mathcal{F}_{r}$-measurable affine transformation $\mathcal{O}_{r}:=\mathcal{O}_{\gamma_{v}\left(\tau_{v}(r)\right)}$ on the event $\left\{\tau_{v}(r)<\infty\right\}$. The map $\mathcal{O}_{r}$ rotates and translates $\mathbb{R}^{d}$ so that at the frontier time $\tau_{v}(r)$, the transformed geodesic is sitting at the origin with the former ball $B^{\mathrm{E}}(0, r)$ contained entirely in the left half-space. We define the random transformation $\mathcal{O}_{r}$ on the space $\Omega=C^{2+\alpha}\left(\mathbb{R}^{d}, \mathrm{SPD}\right)$ by

$$
\left(\mathcal{O}_{r} g\right)(u):=g\left(\mathcal{O}_{r} u\right), \quad u \in \mathbb{R}^{d} .
$$

If we consider a particle traveling along the geodesic $\gamma_{v}$, then by adopting the point of view of the particle, $\Theta_{r} g$ is the environment the particle sees at time $\tau_{v}(r)$. The left half-space represents the "past" of the particle's trajectory, and the right halfspace the "future." The transformation $\mathcal{O}_{r}$ is a random shift, followed by a random rotation. Consequently, the random measure $\mathbb{P} \circ \mathcal{O}_{r}^{-1}$ on $\Omega$ is absolutely continuous with respect to $\mathbb{P}$, as in Section 2.3.

Claim 2.13. Fix $v \in S^{d-1}$. With probability one, for all $r \geq 0$ the random measure $\mathbb{P} \circ \mathcal{O}_{r}^{-1}$ on $\Omega$ is absolutely continuous with respect to $\mathbb{P}$. That is, there exists a family

\footnotetext{
${ }^{1}$ For example, let $\mathcal{O}_{x}^{\text {trans }}$ be the translation which sends 0 to $|x| \mathrm{e}_{1}$, and let $\mathcal{O}_{x}^{\text {rot }}$ be the identity transformation if $x$ is parallel to $\mathrm{e}_{1}$; otherwise, let $\mathcal{O}_{x}^{\text {rot }}$ be the rotation which fixes the $(d-2)$ dimensional space $\operatorname{span}\left\{\mathrm{e}_{1}, x\right\}^{\perp}$, and rotates the vector $\mathrm{e}_{1}$ in the plane $\operatorname{span}\left\{\mathrm{e}_{1}, x\right\}$ to be parallel to $x$. Define $\mathcal{O}_{x}=\mathcal{O}_{x}^{\text {rot }} \mathcal{O}_{x}^{\text {trans }}$.
} 
of measurable functions $\rho_{r}: \Omega \rightarrow \mathbb{R}$ so that for all measurable $f: \Omega \rightarrow \mathbb{R}$,

$$
\int_{\Omega} f\left(\mathcal{O}_{r} g\right) \mathrm{d} \mathbb{P}(g)=\int_{\Omega} f(g) \rho_{r}(g) \mathrm{d} \mathbb{P}(g) .
$$

This should follow from Claim 2.3, where we must account for the stopping time $\tau_{v}(r)$, as well as the random rotation.

For $\rho=2 \xi / \beta$ as above, let $B_{\infty}=B^{\mathrm{E}}(0, \rho) \cap\left\{x: x^{1} \leq 0\right\}$ be the closed left half-ball of radius $\rho$, and let

$$
B_{r}=B^{\mathrm{E}}(0, \rho) \cap B^{\mathrm{E}}\left(-r \mathrm{e}_{1}, r\right)
$$

be the part cut out of $B_{\infty}$ by the large ball $B^{\mathrm{E}}\left(-r \mathrm{e}_{1}, r\right)$. Fix $\eta>0$, and define the cone $C$ in the right half-space by

$$
C=\left\{x \in \mathbb{R}^{d}: 0 \leq x^{1} \leq \eta \text { and } \sqrt{\left(x^{2}\right)^{2}+\cdots+\left(x^{d}\right)^{2}} \leq \rho x^{1}\right\} .
$$

If $\phi$ denotes the angle of of the cone $C$ from the horizontal axis, then $\cos \phi=\beta / 2$. Thus $\phi$ is strictly greater than $\theta=\cos ^{-1} \beta$, since cosine is decreasing.

Write $W=B_{\infty} \cup C$. Note that the only points in the left half-space which are Euclidean distance less than $\xi$ away from $C$ are those in $B_{\infty}$. Conditioned on the left half-space, the metric $\left.g\right|_{C}$ in the cone depends only on the metric $\left.g\right|_{B_{\infty}}$ in the half-ball. This is an important point which we exploit in the proof of Claim 4.2 to show that there is a Markov Property of the metric at frontier times.

Define $\Theta_{r}: \Omega \rightarrow C^{2+\alpha}(W, \mathrm{SPD})$ by

$$
\left(\Theta_{r} g\right)(u)=g\left(\mathcal{O}_{r} u\right), \quad u \in W .
$$

Thus $\Theta_{r} g$ is the metric in the neighborhood of $\gamma_{v}\left(\tau_{v}(r)\right)$, rotated and translated to lie at the origin. Let

$$
\eta_{r}: C^{2+\alpha}(W, \mathrm{SPD}) \rightarrow C^{2}\left(B_{r}, \mathrm{SPD}\right)
$$

be the restriction-and-inclusion map, defined by $\left(\eta_{r} x\right)(u)=x(u)$ for $u \in B_{r}$. The map $\eta_{r} \Theta_{r}: \Omega \rightarrow U_{r}$ is $\mathcal{F}_{r}$-measurable. 
Claim 2.14. Let $v \in S^{d-1}$, and let $\left\{\Theta_{r}\right\}$ be the family of $\mathcal{F}_{r}$-adapted random maps as defined in (2.7). If $U \subseteq C^{2+\alpha}(W, \mathrm{SPD})$ is open, then there exist non-random $p>0$ and $r_{0}>0$ such that if $R \geq r_{0}$ is a frontier of $\gamma_{v}$ and $\eta_{R} \Theta_{R} g \in \eta_{R} U$, then

$$
\mathbb{P}\left(\Theta_{R}^{-1} U \mid \mathcal{F}_{R}\right)>p
$$

The event $\left\{\eta_{R} \Theta_{R} g \in \eta_{R} U\right\}$ is simply that the part of the metric $g$ contained in $B_{R}^{\mathrm{E}}$ is compatible with the event $\Theta_{R}^{-1} U$. In the sequel, this event will be implied by the estimate $(2.3)$.

We sketch the proof of this claim, which involves some tools coming from probability in Banach spaces and developed in [LaG10]. Assumption 2.1.d of this model was that $\mathbb{P}$ is absolutely continuous with respect to a Gaussian measure, which implies that the disintegration (i.e. regular conditional probability) satisfies certain continuity properties [LaG10, Theorem 11]. The Arzelà-Ascoli theorem [Fol99] implies that the set of metrics for which (2.3) holds is compact in the $C^{2}$-norm. This gives us a positive lower bound for the event to occur. The proof is technical and can be found in Chapter 4.

\subsection{Construction of a Bump Surface at Frontier Times and Proof of Main Result}

Consider the cone $C$ as defined in (2.6) as a manifold with boundary. Let $Z=$ $C^{2}(C, \mathrm{SPD})$ be the space of $C^{2}$-Riemannian metrics on $C$. Let $\phi$ be the angle of the cone at 0 , so that $\tan \phi=\rho / \xi=2 / \beta$, and $\phi$ is strictly greater than $\theta=\cos ^{-1} \beta$. Consequently, if a geodesic $\gamma$ starts at the origin with initial rightward direction within angle $\theta$ of $\mathrm{e}_{1}$, that is,

$$
\dot{\gamma}^{1}(0) \geq|\dot{\gamma}(0)| \cos \theta=|\dot{\gamma}(0)| \beta
$$

then $\gamma(t)$ is in the interior of the cone for small, positive time $t$. 
Let $Y=C^{2}\left(B_{\infty}, \mathrm{SPD}\right)$ be the space of $C^{2}$-Riemannian metrics on the half-ball $B_{\infty}$, defined in (2.5) (this is the space $Y_{\infty}$ as defined in Chapter 4 ). The set $\Gamma=\{g \in$ $\left.Y:\|g\|_{C^{2+\alpha}}+1 / \lambda \leq h\right\}$ is compact in $Y$ by the Arzelà-Ascoli theorem [Fol99].

Claim 2.15. There exists a continuous map $b: \Gamma \rightarrow X$ and $\epsilon>0$ such that if $\left\|g-b\left(\left.g\right|_{B_{\infty}}\right)\right\|_{Z}<\epsilon$, then for all geodesics $\gamma$ starting at 0 with initial directions within an angle $\theta$ of $\mathrm{e}_{1}$, there exists a point $x$ in the interior of $C$ such that 0 and $x$ are conjugate points along $\gamma$.

For each $g \in \Gamma$, the function $b(g): C \rightarrow \mathrm{SPD}$ is a Riemannian metric on the cone $C$, which we call a "bump metric." All the geodesics which pass over the bump develop conjugate points [Lee97] and lose the minimization property. While we will see this exact Riemannian manifold with probability zero, the loss of minimization persists under small perturbations of the metric.

This construction has two elements: first that we can construct a Riemannian metric $\tilde{g}:=b(g)$ such that the geodesics remain in the cone $C$ and develop conjugate points, and that this is stable under a uniform perturbation $\epsilon$ of the metric. We have not yet completed the construction with all the technical details, but we include the sketch of our argument here. The cone $C$ meets the half-ball $B_{\infty}$ at the origin, so the Riemannian metric $\tilde{g}$ must agree at 0 with $g$ up to second derivatives. Since $\Gamma$ is compact, these derivatives are all bounded. Other than this condition, we have absolute freedom to choose a Riemannian metric which does whatever we want in $C$.

Let $\tilde{\Gamma}_{i j}^{k}$ be the Christoffel symbols [Lee97] for the Riemannian metric $\tilde{g}$, so that the geodesic equation is

$$
\ddot{\gamma}^{k}=-\tilde{\Gamma}_{i j}^{k} \dot{\gamma}^{i} \dot{\gamma}^{j}
$$

where we follow the Einstein summation convention and sum over the repeated indices $i$ and $j$. In particular, for the first coordinate

$$
\ddot{\gamma}^{1}=-\tilde{\Gamma}_{i j}^{1} \dot{\gamma}^{i} \dot{\gamma}^{j}
$$


As a geodesic approaches the boundary of the cone, we want it to be accelerated rightward, so we want the Christoffel symbols $\tilde{\Gamma}_{i j}^{1}$ to be negative and very large near the boundary. Once we guarantee that the geodesics are moving roughly parallel and to the right, we smooth the metric out into a spherical metric. This is the origin of the name "bump": the attached Riemannian manifold begins with arbitrary (but bounded) positive, zero or negative curvature at the origin, then as geodesics follow the manifold the curvature becomes constant and positive. It is the presence of positive curvature which forces geodesics to develop conjugate points, after which they are not minimizing [Lee97].

Conjugate points occur when the solution to the Jacobi equation [Lee97] along a geodesic vanishes twice. The Jacobi equation is a differential equation with coefficients comprised of the second derivatives of the metric $\tilde{g}$. Consequently, zeros to solutions are stable under small $C^{2}$ perturbations of the metric. For each $y \in \Gamma$, let $\epsilon(y)>0$ be the maximum such perturbation such that the consequence of Claim 2.15 holds. This should be a continuous function of $y$ in the compact set $\Gamma$, hence the minimum $\epsilon=\inf _{y \in \Gamma} \epsilon(y) 0$ is non-zero.

Lemma 2.16. The set $U \subseteq X$ defined by

$$
U=\left\{g \in X:\left\|\left.g\right|_{C}-b\left(\eta_{\infty} g\right)\right\|_{Z}<\epsilon\right\}
$$

is open in $X$.

Proof. The function $f: X \rightarrow \mathbb{R}$ defined by

$$
f(g)=\left\|\left.g\right|_{C}-b\left(\eta_{\infty} g\right)\right\|_{Z}
$$

is continuous, and $U=f^{-1}((-\infty, \epsilon))$.

Finally, we can prove the main result of the paper, and show that $v \in \mathcal{V}$ with probability zero. 
Proof of Claim 2.7. Let $v \in S^{d-1}$. If there is no sequence of frontiers $R_{k} \uparrow \infty$ along $\gamma_{v}$, then $v \notin \mathcal{V}$ by Corollary 2.12. Suppose that the event does hold, and let $R_{k}$ be the sequence of frontiers. Let $U$ be as in the preceding lemma, so if any of the events $\Theta_{R_{k}}^{-1} U$ occur then the geodesic $\gamma_{v}$ is not minimizing.

For all $k$,

$$
\mathbb{P}\left(\bigcap_{k^{\prime}=1}^{k}\left(\Theta_{R_{k^{\prime}}}^{-1} U\right)^{c} \mid \mathcal{F}_{R_{k}}\right) \leq(1-p)^{k} .
$$

Thus with probability one, the event $\Theta_{R_{k}}^{-1} U$ occurs for some $k$. 


\section{CHAPTER 3 \\ Proof of THEOREM 2.10}

In this Chapter we prove Theorem 2.10. Corollary 3.1 is a summary of some results from [LW10]. We apply those results in the proof of Lemma 3.2, which controls the Euclidean arc length of a minimizing geodesic. The key assumption is that $\Lambda / \lambda-$ the ratio of the largest eigenvalue of the Riemannian metric $g$ in a unit cube to the smallest eigenvalue - is a random variable with strong tail decay properties. This means that for most cubes it passes through, a minimizing geodesic will not wiggle too much.

We recall some notation from [LW10]. For $z \in \mathbb{Z}^{d}$, we write $z=\left(z^{1}, \ldots, z^{d}\right)$. We say that $z, z^{\prime} \in \mathbb{Z}^{d}$ are $*$-adjacent if $\max _{1 \leq i \leq d}\left(z-z^{\prime}\right)^{i} \leq 1$. The $*$-lattice is the graph with vertex set $\mathbb{Z}^{d}$, and edge set given by $*$-adjacency; that is, the usual lattice $\mathbb{Z}^{d}$ along with all the diagonal edges.

We say that a set $\Gamma \subseteq \mathbb{Z}^{d}$ is $*$-connected if for all $z, z^{\prime} \in \Gamma$, there is a path from $z$ to $z^{\prime}$ along the $*$-lattice which remains in the set $\Gamma$. Technically, that there is a finite sequence of $*$-adjacent points beginning with $z$ and ending with $z^{\prime}$, all contained in $\Gamma$.

Let $X_{z}$ be a stationary, non-negative random field on the $*$-lattice with finite-range dependence, and with a finite moment-generating function

$$
M(r)=\mathbb{E}\left[\mathrm{e}^{r X}\right]<\infty \quad \text { for all } r \in \mathbb{R}
$$

The finite-range dependence means that there exists $\xi>0$ such that if $\left|z-z^{\prime}\right| \geq \xi$, then $X_{z}$ and $X_{z^{\prime}}$ are independent. We write

$$
X(\Gamma)=\sum_{z \in \Gamma} X_{z}
$$


Assumption c implies that $\Lambda$ and $\Lambda / \lambda$ have finite moment-generating functions and satisfy (3.1). Since $\lambda<\Lambda, \lambda$ also satisfies (3.1).

\section{Corollary 3.1.}

a) For $\mu$ as in Corollary 2.2, with probability one, there exists $M_{1}>0$ such that if $|x| \geq M_{1}$, then $d(0, x) \leq 2 \mu|x|$.

b) Suppose that $X_{z}$ is stationary and positive, and satisfies finite-range dependence and (3.1). For any $A>0$ there is a non-random $B>0$ such that, with probability one, there exists $N>0$ such that for all $n \geq N$, if $\Gamma$ is a $*$-connected set containing the origin and $X(\Gamma) \leq A n$, then $|\Gamma| \leq B n$.

c) Suppose that $X_{z}$ is stationary and non-negative, and satisfies finite-range dependence and (3.1). For any $B>0$ there is a non-random $C>0$ such that, with probability one, there exists $N>0$ such that for all $n \geq N$, if $\Gamma$ is a $*$-connected set containing the origin and $|\Gamma| \leq B n$, then $X(\Gamma) \leq C n$.

Proof. Part (a) is implied by Theorem 2.2.a. Parts (b) and (c) are Lemmas 2.2 and 2.3 of [LW10], respectively, applied to the constant sequence $a_{n} \equiv 0$.

Lemma 3.2. There exists a non-random $D \geq 1$ such that, with probability one, there exists $M>0$ such that if $|x| \geq M$ and $\gamma$ is a length-minimizing geodesic connecting 0 to $x$, then

$$
|x| \leq L(\gamma) \leq D|x|
$$

where $L(\gamma)$ denotes the Euclidean length of $\gamma$ between 0 and $x$.

Proof. The lower estimate $|x| \leq L(\gamma)$ is trivial, since $\gamma$ has Euclidean length at least that of the straight line path from 0 to $x$.

By Corollary 3.1.a, with probability one, there exists $M_{1}>0$ such that if $|x|>M_{1}$, then

$$
d(0, x) \leq 2 \mu|x|
$$


Apply Corollary 3.1.b to $A=8 \mu$ and $X_{z}=\lambda_{z}$. Thus there exists a non-random $B>0$ such that, with probability one, there exists $N_{1}>0$ such that for all $n \geq N_{1}$, if $\Gamma$ is a finite $*$-connected set which contains the origin and $\lambda(\Gamma) \leq 8 \mu n$, then $|\Gamma| \leq B n$.

By Assumption c, $\Lambda_{z} / \lambda_{z}$ has a finite moment-generating function. Apply Corollary 3.1.c to the above $B$ and $X_{z}=\Lambda_{z} / \lambda_{z}$. Thus there exists a non-random $C>0$ such that, with probability one, there exists $N_{2}>0$ such that for all $n \geq N_{2}$, if $\Gamma$ is a finite *-connected set which contains the origin and $|\Gamma| \leq B n$, then $(\Lambda / \lambda)(\Gamma) \leq C n$.

Set $D=\frac{3^{d} B}{2}+2 C \sqrt{d}$, and let $|x| \geq \max \left\{M_{1}, N_{1}, N_{2}, 1\right\}$. Let $n$ be the smallest integer greater than $|x|$; we will later use the trivial estimate $n \leq 2|x|$. Let $\gamma$ be a length-minimizing geodesic between 0 and $x$. Since $\gamma$ connects the origin to a point Euclidean distance $|x|$ away, $L(\gamma) \geq|x|$. Define the discrete set

$$
\Gamma=\left\{z \in \mathbb{Z}^{d}: L\left(\gamma \cap C_{z}\right) \geq 1 / 4\right\}
$$

That is, $z \in \Gamma$ if $\gamma$ spends at least Euclidean length $1 / 4$ in the unit cube $C_{z}$. The set $\Gamma$ is $*$-connected; see the discussion following (2.8) of [LW10]. Clearly, $0 \in \Gamma$.

Since $\gamma$ is length-minimizing,

$$
R(\gamma)=d(0, x) \leq 2 \mu|x| \leq 2 \mu n
$$

Furthermore, by summing $\lambda_{z}$ over the points of $\Gamma$, we get an upper bound using $R(\gamma)$ :

$$
\frac{1}{4} \lambda(\Gamma) \leq \sum_{z \in \Gamma} L\left(\gamma \cap C_{z}\right) \lambda_{z} \leq \sum_{z \in \Gamma} R\left(\gamma \cap C_{z}\right) \leq R(\gamma) \leq 2 \mu n
$$

Thus, $\lambda(\Gamma) \leq 8 \mu n$, hence

$$
|\Gamma| \leq B n
$$

and $(\Lambda / \lambda)(\Gamma) \leq C n$.

In each cube $C_{z}$, we can estimate the Euclidean length of $\gamma$ using $\Lambda_{z} / \lambda_{z}$ :

$$
L\left(\gamma \cap C_{z}\right) \lambda_{z} \leq R\left(\gamma \cap C_{z}\right) \leq \Lambda_{z} \sqrt{d}
$$


So

$$
L\left(\gamma \cap C_{z}\right) \leq \frac{\Lambda_{z}}{\lambda_{z}} \sqrt{d}
$$

Define the set $\hat{\Gamma}$ consisting of $\Gamma$ and all neighboring points on the $*$-lattice:

$$
\hat{\Gamma}=\left\{z \in \mathbb{Z}^{d}: \exists z^{\prime} \in \Gamma \text { s.t. } z \text { and } z^{\prime} \text { are } * \text {-adjacent }\right\} \supset \Gamma \text {. }
$$

The geodesic $\gamma$ is completely contained in union of the cubes with centers $\hat{\Gamma}$. The geodesic can get contributions to Euclidean length from the cubes with centers $z \in$ $\hat{\Gamma} \backslash \Gamma$, but only up to $1 / 4$ and there are fewer than $3^{d}|\Gamma| \leq 3^{d} B n$ of such cubes. Thus $L(\gamma) \leq \sum_{z \in \hat{\Gamma} \backslash \Gamma} L\left(\gamma \cap C_{z}\right)+\sum_{z \in \Gamma} L\left(\gamma \cap C_{z}\right) \leq \frac{3^{d} B}{4} n+\sqrt{d} \sum_{z \in \Gamma} \frac{\Lambda_{z}}{\lambda_{z}} \leq \frac{3^{d} B}{4} n+\sqrt{d} C n=\frac{1}{2} D n$, since $D=\frac{3^{d} B}{2}+2 C \sqrt{d}$. Since $n \leq 2|x|$, the proof is complete.

Let $\beta=1 / 2 D<1$, and let $\theta \in(0, \pi / 2)$ be the angle such that $\cos \theta=\beta$. For $v \in \mathcal{V}$, consider the length-minimizing geodesic $\gamma_{v}$, and suppose that it is parametrized by Euclidean arc length $l$. Write $r_{v}(l)=\left|\gamma_{v}(l)\right|$. Define the set of Euclidean frontier times of $\gamma_{v}$ to be

$$
F_{v}=\left\{l: \dot{r}_{v}(l)>\beta \text { and } r_{v}(l)=\sup _{l^{\prime} \leq l} r_{v}\left(l^{\prime}\right)\right\} .
$$

In the next lemma, we show that the set of Euclidean frontier times takes up a non-zero fraction of the Euclidean length of $\gamma_{v}$.

Lemma 3.3. With probability one, for all $v \in \mathcal{V}$, the set of Euclidean frontier times $F_{v} \subseteq[0, \infty)$ comprises right-open intervals and is unbounded. Furthermore, there exists non-random $\delta>0$ such that, with probability one, there exists $L>0$ such that if $l \geq L$, then

$$
\operatorname{Leb}\left(F_{v} \cap[0, l]\right) \geq \delta l
$$

for all $v \in \mathcal{V}$. 
Proof. We first argue that $F_{v}$ is right-open. Suppose $l \in F_{v}$. Since $\dot{r}$ is continuous, there exists $\epsilon>0$ such that if $h \in[0, \epsilon)$, then $\dot{r}_{v}(l+h)>\beta$. Since $r_{v}(l)=\sup _{l^{\prime} \leq l} r_{v}\left(l^{\prime}\right)$ and $r$ is strictly increasing on $[l, l+\epsilon), r_{v}(l+h)$ is the new supremum. Thus $[l, l+\epsilon) \subseteq$ $F_{v}$.

Let $D$ and $M$ be as in Lemma 3.2. Let $K=B^{\mathrm{E}}(0, M)$ be the Euclidean ball of (random) radius $M$. By Theorem 2.9, with probability one, all minimizing geodesics escape $K$ in uniform time: there exists $L$ such that if $l \geq L$ and $v \in \mathcal{V}$, then $r_{v}(l)=\left|\gamma_{v}(l)\right| \geq M$, hence

$$
r_{v}(l) \leq l \leq D r_{v}(l)
$$

Let $\delta=1 /(2 D-1)$. Write $S=\left\{l: r_{v}(l)=\sup _{l^{\prime} \leq l} r_{v}\left(l^{\prime}\right)\right\}$ for the times $l$ at which $r_{v}(l)$ attains the supremum, so that we can decompose the non-frontier times $F_{v}^{c}$ by

$$
F_{v}^{c}=(\{0 \leq \dot{r} \leq \beta\} \cap S) \cup S^{c} .
$$

If $l \geq L$, then the fundamental theorem of calculus implies that

$$
D^{-1} l \leq r_{v}(l)=\int_{0}^{l} \dot{r}=\int_{F_{v} \cap[0, l]} \dot{r}+\int_{\{0 \leq \dot{r} \leq \beta\} \cap S \cap[0, l]} \dot{r}+\int_{S^{c} \cap[0, l]} \dot{r} .
$$

Since $f_{v}(l):=\sup _{l^{\prime} \leq l} r_{v}\left(l^{\prime}\right)-r_{v}(l)$ is continuous, $S^{c}=f_{v}^{-1}((0, \infty))$ is open, hence a union of open intervals. Let $I$ be a maximal subinterval of $S^{c}$. The curve $\gamma_{v}$ is transient by Theorem 2.9 so $f(l)=0$ for arbitrarily large $l$; this implies that $I$ is bounded. At both endpoints of $I$, the function $r$ equals $\sup r$, so the third integral of (3.5) vanishes.

Write $b(l)=\operatorname{Leb}\left(F_{v} \cap[0, l]\right)$; we must show $b(l) \geq \delta l$. Since the geodesic is parametrized by Euclidean length, $\dot{r} \leq 1$. We use this to estimate the first integral of (3.5); for the second integral, we use $\dot{r} \leq \beta$. Thus

$$
D^{-1} l \leq 1 \cdot b(l)+\beta \cdot(l-b(l))+0 .
$$

Since $\beta=1 / 2 D$ and $\delta=1 /(2 D-1)$, by rewriting this expression, we have $b(l) \geq \delta l$ as desired. 
Proof of Theorem 2.10. Suppose that geodesics are parametrized by Euclidean length. For $v \in S^{d-1}$, let $t_{v}(l)$ be the change in parametrization to Riemannian arc length along $\gamma_{v}$, and let $r_{v}(l)=\left|\gamma_{v}(l)\right|$. It suffices to prove that for all $v \in \mathcal{V}$, there exists a sequence $l_{k} \uparrow \infty$ such that the conclusions of Theorem 2.10 hold for the sequence $t_{k}:=t_{v}\left(l_{k}\right)$. The metric is complete with probability one, so $l_{k} \uparrow \infty$ implies that $t_{k} \uparrow \infty$.

Let $v \in \mathcal{V}$, and fix $l \in F_{v}$. We first prove that the angle between $\gamma_{v}(l)$ and $\dot{\gamma}_{v}(l)$ is less than $\theta:=\cos ^{-1} \beta$. This follows quickly from the definition of frontier times and elementary trigonometry. Since $\gamma_{v}$ is parametrized by Euclidean length, $\left|\dot{\gamma}_{v}(l)\right|=1$. Since $l$ is a frontier time, $\dot{r}_{v}(l) \geq \beta$ : the projection of $\dot{\gamma}(l)$ onto the direction $\gamma(l)$ is at least $\beta$. Consequently, the angle between $\dot{\gamma}(l)$ and $\gamma(l)$ is at $\operatorname{most} \theta$, where $\cos \theta=\beta$.

Fix $\rho>0$, and let $m$ be the minimum number of cubes $C_{z}$ which can cover any Euclidean ball of radius $\rho$. Let $B$ be as in Lemma 3.2. Write $\tilde{\rho}=\rho+\sqrt{d}$.

Let $v \in \mathcal{V}$. Define an increasing sequence of frontier times $l_{j} \in F_{v}$ and balls $B_{j} \subseteq \mathbb{R}^{d}$ as follows. Let $l_{0}=0$ and

$$
l_{j}=\inf \left\{l \in F_{v}: l>l_{j-1} \text { and }\left|\gamma(l)-\gamma\left(l_{j^{\prime}}\right)\right| \geq 2 \tilde{\rho} \text { for } j^{\prime}<j\right\}
$$

Define the ball $B_{j}=B^{\mathrm{E}}\left(\gamma\left(l_{j}\right), \rho\right)$ of radius $\rho$ centered at $\gamma\left(l_{j}\right)$, and let

$$
\tilde{B}_{j}=\left\{z \in \mathbb{Z}^{d}: B_{j} \cap C_{z} \neq \emptyset\right\}
$$

be the centers of the cubes $C_{z}$ which form a discrete cover of $B_{j}$, so $\left|\tilde{B}_{j}\right| \leq m$. The discrete sets $\tilde{B}_{j}$ are disjoint, since two distinct $\rho$-balls $B_{j}$ are separated by distance at least $\sqrt{d}$.

Lemma 3.4. Let $\delta$ and $L$ be as in Lemma 3.3, and let $A=\frac{8 \tilde{\rho}}{\beta \delta}$. If $l_{j} \geq L$ then

$$
l_{j} \leq A j
$$

Proof. Clearly, the balls $B\left(\gamma\left(l_{j}\right), 4 \tilde{\rho}\right)$ of larger radius $4 \tilde{\rho}$ cover the image under $\gamma$ of 
all frontier times $F_{v}$ :

$$
F_{v} \subseteq \bigcup_{j^{\prime}=1}^{\infty}\left\{l \in F_{v}:\left|\gamma(l)-\gamma\left(l_{j^{\prime}}\right)\right| \leq 4 \tilde{\rho}\right\}=: \bigcup_{j^{\prime}=1}^{\infty} I_{j^{\prime}}
$$

hence

$$
\operatorname{Leb}\left(F_{v} \cap\left[0, l_{j}\right]\right) \leq \sum_{j^{\prime}=1}^{j} \operatorname{Leb}\left(I_{j^{\prime}}\right)
$$

On $I_{j}$, the maximum distance to the origin $\sup _{l^{\prime} \leq l} r_{v}\left(l^{\prime}\right)$ can grow by at most $8 \tilde{\rho}$, the diameter of the ball $B^{\mathrm{E}}\left(\gamma\left(l_{j}\right), 4 \tilde{\rho}\right)$. Thus by the fundamental theorem of calculus,

$$
8 \tilde{\rho} \geq \int_{I_{j}} \dot{r} \geq \beta \operatorname{Leb}\left(I_{j}\right) .
$$

If $l_{j} \geq L$, then Lemma 3.3 implies that $\delta l_{j} \leq \operatorname{Leb}\left(F_{v} \cap\left[0, l_{j}\right]\right)$. Thus

$$
l_{j} \leq \frac{1}{\delta} \sum_{j^{\prime}=1}^{j} \operatorname{Leb}\left(I_{j^{\prime}}\right) \leq \frac{8 \tilde{\rho}}{\delta \beta} j=A j
$$

Let

$$
W_{j}=\left\{y \in \mathbb{R}^{d}:|y-\gamma(l)| \leq \rho \text { for some } l \in\left[0, l_{j}\right]\right\}
$$

be the $\rho$-neighborhood of $\left.\gamma\right|_{\left[0, l_{j}\right]}$. Let

$$
\tilde{\Gamma}_{j}=\left\{z \in \mathbb{Z}^{d}: C_{z} \cap W_{j} \neq \emptyset\right\}
$$

be the centers of the cubes $C_{z}$ which cover $W_{j}$. Note that $\tilde{B}_{j^{\prime}} \subseteq \tilde{\Gamma}_{j}$ for all $j^{\prime} \leq j$.

Lemma 3.5. There exists non-random $B^{\prime}>0$ and there exists $J_{1}>0$ such that if $j \geq J_{1}$, then

$$
\left|\tilde{\Gamma}_{j}\right| \leq B^{\prime} j
$$

Proof. Let $L$ be as in Lemma 3.3. Let

$$
\Gamma_{j}=\left\{z \in \mathbb{Z}^{d}:\left.\gamma\right|_{\left[0, l_{j}\right]} \cap C_{z} \neq \emptyset\right\}
$$


be the centers of the cubes $C_{z}$ which the curve $\left.\gamma\right|_{\left[0, l_{j}\right]}$ meets. As in Lemma 3.2, there exists non-random $B>0$ and there exists $L_{1}>0$ such that if $l_{j} \geq L_{1}$, then $\left|\Gamma_{j}\right| \leq B l_{j}$. Let $J_{1}$ be the minimum $j$ such that $l_{j} \geq \max \left\{L, L_{1}\right\}$, and suppose $j \geq J_{1}$. By (3.6), $l_{j} \leq A j$. Let $B^{\prime}=m B A$, so

$$
\left|\tilde{\Gamma}_{j}\right| \leq m\left|\Gamma_{j}\right| \leq m B l_{j} \leq m B A j=B^{\prime} j .
$$

Now let $h \in(0, \infty)$, and let $A_{z}^{h}$ be the event that

$$
\|g\|_{C^{2+\alpha}\left(C_{z}\right)}+\frac{1}{\lambda\left(C_{z}\right)}>h
$$

Let $X_{z}^{h}=1\left(A_{z}^{h}\right)$ be the indicator function of the event $A_{z}^{h}$. Since the family $X_{z}^{h}$ only takes the values 0 and 1 , it is bounded hence has a finite moment-generating function.

Apply Corollary 3.1.c to the $B^{\prime}$ from Lemma 3.5 and the family $X_{z}^{h}$. Thus there exists a non-random $C(h)>0$ (depending on $h$ ) such that, with probability one, there exists $J_{2}>0$ such that for all $j \geq J_{2}$, if $\Gamma$ is a finite $*$-connected set which contains the origin and $|\Gamma| \leq B^{\prime} j$, then $X^{h}(\Gamma) \leq C(h) j$.

With probability one, the metric $g$ is $C^{2+\alpha}$ and positive everywhere. Thus for every $z \in \mathbb{Z}^{d}$,

$$
\lim _{h \rightarrow \infty} \mathbb{P}\left(A_{z}^{h}\right)=0 .
$$

Consequently, $C(h) \rightarrow 0$ as $h \rightarrow \infty$. Choose a value of $h$ large enough so that

$$
C(h)<\frac{1}{2}
$$

Let $j \geq \max \left\{J_{1}, J_{2}\right\}$. By the above lemma, $\left|\tilde{\Gamma}_{j}\right| \leq B^{\prime} j$ so

$$
X^{h}\left(\tilde{\Gamma}_{j}\right) \leq C(h) j<\frac{j}{2} .
$$

That is, the number of points $z \in \tilde{\Gamma}_{j}$ for which that the event $A_{z}^{h}$ occurs is fewer than $j / 2$. There are $j$ disjoint sets $\left\{\tilde{B}_{j^{\prime}}\right\}$ contained in $\tilde{\Gamma}_{j}$; consequently, there are at least $j / 2$ balls $B_{j_{k}}$ such that

$$
\|g\|_{C^{2+\alpha}\left(B_{j_{k}}\right)}+\frac{1}{\lambda\left(B_{j_{k}}\right)} \leq h
$$


As $j \rightarrow \infty$, we may choose infinitely many $j_{k} \rightarrow \infty$. This proves Theorem 2.10 . $\square$ 


\section{Chapter 4}

\section{Sketch of Proof of Claim 2.14}

Let $B_{r}$ be the half-ball and $C$ be the cone defined as in (2.5) and (2.6), respectively, and let $W=B_{\infty} \cup C$. Recall that $\Omega=C^{2+\alpha}\left(\mathbb{R}^{d}, \mathrm{SPD}\right)$. Write Sym for the space of symmetric $d \times d$ real matrices, and consider the Banach spaces

$$
X=C^{2+\alpha}(W, \text { Sym }) \quad \text { and } \quad Y_{r}=C^{2}\left(B_{r}, \text { Sym }\right), \quad r \leq \infty
$$

equipped with the $C^{2+\alpha}$ and $C^{2}$ norms, respectively. The set inclusions $B_{r} \subseteq B_{\infty} \subseteq$ $W \subseteq \mathbb{R}^{d}$ induce restriction-and-inclusion maps

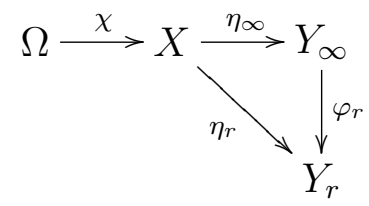

We need to account for the parameter $r$ in our maps, since the region we will be conditioning on later on will be cut out from the large ball $B^{\mathrm{E}}\left(-r \mathrm{e}_{1}, r\right)$.

Let $\mathbb{P}_{X}=\mathbb{P} \circ \chi^{-1}$ be the push-forward of the probability measure $\mathbb{P}$ on $X$. Similarly, let $\mathbb{P}_{Y_{r}}=\mathbb{P}_{X} \circ \eta_{r}^{-1}$ be the push-forward probability measures on $Y_{r}$ for $r \leq \infty$. When there is no ambiguity we will write $\mathbb{P}$ for $\mathbb{P}_{X}$. These measures satisfy the change-ofvariable equations

$$
\int_{\Omega} f(\chi g) \mathrm{d} \mathbb{P}(g)=\int_{X} f(x) d \mathbb{P}_{X}(x) \quad \text { and } \quad \int_{X} g\left(\eta_{r} x\right) d \mathbb{P}_{X}(x)=\int_{Y_{r}} g(y) d \mathbb{P}_{Y_{r}}(y)
$$

for any measurable functions $f: X \rightarrow \mathbb{R}$ and $g: Y_{r} \rightarrow \mathbb{R}$.

Assumption 2.1.d implies that there exists a stationary, mean-zero Gaussian measure $\hat{\mathbb{P}}$ on $X$ such that $\mathbb{P}$ is absolutely continuous with respect to $\hat{\mathbb{P}}$ on $X$; and the Radon-Nikodym derivative

$$
\rho(x):=\frac{\mathrm{d} \mathbb{P}}{\mathrm{d} \mathbb{P}}(x)
$$


is bounded and continuous, and is positive exactly on the open subset

$$
X^{0}:=C^{2+\alpha}(W, \mathrm{SPD}) \subseteq X .
$$

This implies that $X^{0}$ has full $\mathbb{P}$-measure. Let $\mathbb{P}_{Y_{r}}=\mathbb{P} \circ \eta_{r}^{-1}$ denote the push-forward measures of $\mathbb{P}$ on the spaces $Y_{r}$. Consequently, the sets

$$
Y_{r}^{0}:=C^{2+\alpha}\left(B_{r}, \mathrm{SPD}\right)=\eta_{r}\left(X^{0}\right) \subseteq Y_{r}
$$

have full $\mathbb{P}_{Y_{r}}$-measure, though not open since the spaces $Y_{r}$ are equipped with the $C^{2}$-norm instead of the $C^{2+\alpha}$-norm.

Proposition 4.1. There exist regular conditional probabilities $\nu_{r}: Y_{r}^{0} \times \mathcal{B}(X) \rightarrow[0,1]$ such that:

a) If $\Gamma \subseteq Y_{r}^{0}$ is compact in $Y_{r}$, and if $y_{n} \in \Gamma$ and $y_{n} \rightarrow y$, then the measures $\nu_{r}\left(y_{n}, \cdot\right)$ converge weakly to $\nu_{r}(y, \cdot)$ on $X$.

b) If $B \subseteq X^{0}$ is open and $y \in Y_{r}^{0} \cap \eta_{r}(B)$, then $\nu_{r}(y, B)>0$.

c) Claim: If $\Gamma \subseteq Y_{\infty}^{0}$ is compact in $Y_{\infty}$ and $B \subseteq X^{0}$ is open, then for all $\epsilon>0$, there exists $R>0$ such that if $r \geq R$, then for all $y \in \Gamma$,

$$
\nu_{r}\left(\varphi_{r} y, B\right) \geq \nu_{\infty}(y, B)-\epsilon .
$$

Proof of a). Let $c: W \times W \rightarrow$ Sym be the matrix-valued covariance function of the Gaussian measure $\hat{\mathbb{P}}$. i.e., if $\omega \in X$ is a realization of $\hat{\mathbb{P}}$, then

$$
c(u, v)=\hat{\mathbb{E}}(\omega(u) \omega(v)),
$$

where the product is matrix multiplication. For $u \in W$, write $c_{u}(\cdot)=c(u, \cdot)$, so $c_{u} \in X$. If $u \in B_{r}$, then $c_{u} \in Y_{r}$.

Let $K: X^{*} \rightarrow X$ be the covariance operator of the Gaussian measure $\hat{\mathbb{P}}$ on $X$, defined by $K f(u)=f\left(c_{u}\right)$. The spaces

$$
\hat{Y}_{r}=\overline{\eta_{r} K \eta_{r}^{*} Y_{r}^{*}} \subseteq Y_{r}
$$


have full $\hat{\mathbb{P}}_{Y_{r}}$-measure. Furthermore, as subspaces of $X$,

$$
\overline{\bigcup K \eta_{r}^{*} Y_{r}^{*}}=\overline{\operatorname{span}_{u \in \bigcup B_{r}}\left\{c_{u}\right\}}=\overline{\operatorname{span}_{u \in B \infty}\left\{c_{u}\right\}}=\overline{K \eta_{\infty}^{*} Y_{\infty}^{*}}
$$

since the family $\left\{c_{u}\right\}$ is equicontinuous for $u \in \bigcup B_{r}$.

Let $u_{0} \in \bigcap B_{r}$. We assume that $\hat{\mathbb{P}}$ is non-degenerate, so $\left\|c\left(u_{0}, u_{0}\right)\right\|_{\text {Sym }}>0$. Let $M=\sup _{u_{\in} B_{\infty}}\left\|c_{u}\right\|_{X} /\left\|c\left(u_{0}, u_{0}\right)\right\|_{\text {Sym }}<\infty$. Since $c$ is stationary,

$$
M_{r}:=\sup _{e \in Y_{r}^{*}} \frac{\left\|K \eta_{r}^{*} e\right\|}{\left\|\eta_{r} K \eta_{r}^{*} e\right\|}=\sup _{u \in B_{r}} \frac{\left\|c_{u}\right\|_{X}}{\left\|c_{u}\right\|_{Y}} \leq \frac{\sup _{u_{\in} B_{\infty}}\left\|c_{u}\right\|_{X}}{\left\|c\left(u_{0}, u_{0}\right)\right\|_{\text {Sym }}}=M<\infty
$$

uniformly for all $r \leq \infty$. On the dense subspace $\eta_{r} K \eta_{r}^{*} Y_{r}^{*}$ of $\hat{Y}_{r}$, define $m_{r}$ : $\eta_{r} K \eta_{r}^{*} Y_{r}^{*} \rightarrow X$ by $y \mapsto \eta_{r}^{-1}(y)$. This linear map has operator norm $M_{r} \leq M<\infty$, hence we may extend $m_{r}$ continuously to all of $\hat{Y}_{r}$.

For all $r \leq \infty$ and $y \in \hat{Y}_{r}$, let $\mathbb{P}_{Y_{r}}^{y}$ be the Gaussian measure on $X$ with mean $m_{r}(y)$ and covariance operator $\hat{K}_{r}=K-K \eta_{r}^{*} m_{r}^{*}$. By Theorem 6 of [LaG10], each $\hat{\mathbb{P}}_{Y_{r}}^{y}$ is a continuous disintegration on $\hat{Y}_{r}$ with respect to the map $\eta_{r}$. That is, $\hat{\mathbb{P}}_{Y_{r}}^{y}$ is a regular conditional probability with respect to $\eta_{r}$, and if $y_{n} \in \hat{Y}_{r}$ and $y_{n} \rightarrow y$, then $\hat{\mathbb{P}}_{Y_{r}}^{y_{n}} \rightarrow \hat{\mathbb{P}}_{Y_{r}}^{y}$ weakly.

In the context of $\mathbb{P} \ll \hat{\mathbb{P}}$, Theorem 11 of [LaG10] implies that $\mathbb{P}_{Y_{r}} \ll \hat{\mathbb{P}}_{Y_{r}}$ with bounded, continuous Radon-Nikodym derivative

$$
\rho_{Y_{r}}(y):=\frac{\mathrm{d} \mathbb{P}_{Y_{r}}}{\mathrm{~d}_{Y_{Y_{r}}}}(y)=\int_{\eta_{r}^{-1}(y)} \rho(x) \mathrm{d} \hat{\mathbb{P}}_{r}^{y}(x) .
$$

The function $\rho_{Y_{r}}$ is positive exactly on the set $Y_{r}^{0}$. Theorem 11 of [LaG10] also implies that the measure $\nu_{r}: Y_{r}^{0} \times \mathcal{B}(X) \rightarrow[0,1]$ defined by

$$
\nu_{r}(y, B)=\int_{B} \frac{\rho(x)}{\rho_{Y_{r}}(y)} \mathrm{d} \hat{\mathbb{P}}_{r}^{y}(x)
$$

is a regular conditional probability for $\mathbb{P}$ and that property (a) holds.

Proof of b). Let $B \subseteq X^{0}$ be open, and let $y \in Y_{r}^{0} \cap \eta_{r}(B)$. Choose $x_{0} \in B$ such that $\eta_{r}\left(x_{0}\right)=y$. The function $\rho$ is positive and continuous at $x_{0}$ and the set $B$ is open so 
there exist $a>0$ and $\delta>0$ such that the open ball $B\left(x_{0}, \delta\right)$ is contained in $B$, and $\rho(x)>a$ on $B\left(x_{0}, \delta\right)$. Gaussian measures assign positive measure to open sets, so

$$
\nu_{r}(y, B)=\int_{B} \frac{\rho(x)}{\rho_{Y_{r}}(y)} \mathrm{d} \hat{\mathbb{P}}_{r}^{y}(x) \geq \frac{a}{\rho_{Y_{r}}(y)} \hat{\mathbb{P}}_{r}^{y}\left(B\left(x_{0}, \delta\right)\right)>0 .
$$

Sketch of proof of c). Define $Y_{r, \infty}=\eta_{\infty} K \eta_{r}^{*} Y_{r}^{*} \subseteq \hat{Y}_{\infty}$. The union of these spaces is dense in $\hat{Y}_{\infty}$, since

$$
\overline{\bigcup Y_{r, \infty}}=\overline{\operatorname{span}_{u \in \bigcup B_{r}}\left\{c_{u}\right\}}=\overline{\operatorname{span}_{u \in B_{\infty}}\left\{c_{u}\right\}}=\hat{Y}_{\infty}
$$

We show now that the operators $m_{r} \varphi_{r}$ converge uniformly to $m_{\infty}$ on $\hat{Y}_{\infty}$. On $Y_{r, \infty}$, the maps $m_{r} \varphi_{r}$ and $m_{\infty}$ are equal, since if $g \in Y_{r}^{*}$,

$$
\left(m_{r} \varphi_{r}-m_{\infty}\right)\left(\eta_{\infty} K \eta_{r}^{*} g\right)=m_{r} \eta_{r} K \eta_{r}^{*} g-m_{\infty} \eta_{\infty} K \eta_{\infty}^{*}\left(\varphi_{r}^{*} g\right)=0
$$

Let $\epsilon>0$, and choose $r_{0}$ such that for all $y \in \hat{Y}_{\infty}$, there is some $y^{\prime} \in Y_{r_{0}, \infty}$ such that $\left\|y-y^{\prime}\right\|_{Y_{\infty}} \leq \epsilon / 2 M$. Then

$\left\|\left(m_{r} \varphi_{r}-m_{\infty}\right)(y)\right\|_{X} \leq\left\|\left(m_{r} \varphi_{r}-m_{\infty}\right)\left(y-y^{\prime}\right)\right\|_{X}+0 \leq\left(\left\|m_{r}\right\|\left\|\varphi_{r}\right\|+\left\|m_{\infty}\right\|\right)\left\|y-y^{\prime}\right\| \leq \epsilon$,

proving that $m_{r} \varphi_{r}$ converges uniformly to $m_{\infty}$.

The Gaussian measure $\hat{\mathbb{P}}_{Y_{\infty}}^{y}$ has mean $m_{\infty}(y)$ and covariance operator $\hat{K}_{\infty}=$ $K-K \eta_{\infty}^{*} m_{\infty}^{*}$, and the Gaussian measure $\hat{\mathbb{P}}_{Y_{r}}^{\varphi_{r} y}$ has mean $m_{r}\left(\varphi_{r} y\right)$ and covariance operator

$$
\hat{K}_{r}=K-K \eta_{r}^{*} m_{r}^{*}=K-K\left(\varphi_{r} \eta_{\infty}\right)^{*} m_{r}^{*}=\hat{K}_{\infty}-K \eta_{\infty}^{*}\left(m_{r} \varphi_{r}-m_{\infty}\right)^{*}
$$

It should follow from standard theory on Gaussian measures [Bog98, IR78] that an approximation statement like (4.2) holds for the Gaussian measures $\hat{\mathbb{P}}_{Y_{\infty}}^{y}$ and $\hat{\mathbb{P}}_{Y_{r}}^{\varphi_{r} y}$. Once we have proved that, estimate (4.2) should follow easily from the explicit construction of $\nu$ from the Gaussian disintegration. 
Let $\chi: \Omega \rightarrow X$ be the restriction-and-inclusion map from $\Omega$ to $X$ and let $\Theta_{r}=$ $\chi \mathcal{O}_{r}: \Omega \rightarrow X$ be the family of $\mathbb{P}$-random maps as defined in (2.7), depending on fixed $v \in S^{d-1}$.

Proposition 4.2 (Markov Property). If $v \in S^{d-1}$ and $f: X \rightarrow \mathbb{R}$ is measurable, then for all $r<\infty$,

$$
\mathbb{E}\left(f \circ \Theta_{r} \mid \mathcal{F}_{r}\right)=\int_{X} f(x) \nu_{r}\left(\eta_{r} \Theta_{r} g, \mathrm{~d} x\right)
$$

on the event $\left\{\tau_{v}(r)<\infty\right\}$ for $\mathbb{P}$-almost every $g \in \Omega$.

Proof. For this proof, we suppose that $r$ is fixed, and consequently drop it from our notation when it is clear. Recall that $\Theta_{r}=\chi \circ \mathcal{O}_{r}$. By Claim 2.13, the measure $\mathbb{P} \circ \mathcal{O}_{r}^{-1}$ is absolutely continuous to $\mathbb{P}$ on the event $\left\{\tau_{v}(r)<\infty\right\}$, so we will first prove a statement analogue to (4.3) without the random transformation $\mathcal{O}_{r}$. After that, we will transform the measure and prove (4.3).

Consider the $\sigma$-algebras

$\mathcal{F}_{B^{\mathrm{E}}}=\sigma\left\{g(x): x \in B^{\mathrm{E}}\left(-r \mathrm{e}_{1}, r\right)\right\}, \quad \mathcal{F}_{B}=\sigma\left\{g(x): x \in B_{r}\right\}, \quad$ and $\quad \mathcal{F}_{W}=\sigma\left\{g(x): x \in B_{r} \cup C\right\}$.

By the construction of $B_{r}$ and $C$, the sets $B^{\mathrm{E}}\left(-r \mathrm{e}_{1}, r\right) \backslash B_{r}$ and $C$ are separated by Euclidean distance at least $\xi$. Thus as Hilbert subspaces of $L^{2}(\Omega, \mathcal{F})$, this implies that

$$
L^{2}\left(\Omega, \mathcal{F}_{B^{\mathrm{E}}}\right) \cap L^{2}\left(\Omega, \mathcal{F}_{W}\right)=L^{2}\left(\Omega, \mathcal{F}_{B}\right)
$$

The random variable $f \chi: \Omega \rightarrow \mathbb{R}$ is $\mathcal{F}_{W}$-measurable, so conditioning it on the $\sigma$ algebra $\mathcal{F}_{B^{\mathrm{E}}}$ reduces to conditioning on $\mathcal{F}_{B}$ :

$$
\mathbb{E}\left(f \chi \mid \mathcal{F}_{B^{\mathrm{E}}}\right)=\mathbb{E}\left(f \chi \mid \mathcal{F}_{B}\right)
$$

Now, we claim that

$$
\mathbb{E}\left(f \chi \mid \mathcal{F}_{B}\right)=\int_{X} f(x) \nu\left(\eta_{r} \chi g, \mathrm{~d} x\right)
$$


for $\mathbb{P}$-almost every $g$. Suppose $A$ is a $\mathcal{F}_{B}$-measurable event. The map $(\eta \chi)^{-1}(\eta \chi)$ : $\mathcal{F} \rightarrow \mathcal{F}$ projects an event onto the coordinates generated by points in $U$; consequently, $(\eta \chi)^{-1}(\eta \chi) A=A$. Thus by applying both change-of-variable formulas (4.1) and the disintegration equation,

$$
\begin{aligned}
\int_{A} \mathbb{E}\left(f \chi \mid \mathcal{F}_{B}\right) \mathrm{d} \mathbb{P}(g) & =\int_{A} f(\chi g) \mathrm{d} \mathbb{P}(g) \\
& =\int_{\chi A} f(x) \mathrm{d} \mathbb{P}_{X}(x) \\
& =\int_{\eta \chi A} \int_{X} f(x) \nu(y, \mathrm{~d} x) \mathrm{d} \mathbb{P}_{Y}(y) \\
& =\int_{A} \int_{X} f(x) \nu(\eta \chi g, \mathrm{~d} x) \mathrm{d} \mathbb{P}(g) .
\end{aligned}
$$

This proves (4.5).

We return to the random-transformation case to prove (4.3). Let $A \in \mathcal{F}_{r}$, and write $A^{\prime}=A \cap\left\{\tau_{v}(r)<\infty\right\}$. We claim that

$$
\int_{A^{\prime}} \mathbb{E}\left(f \circ \Theta_{r} \mid \mathcal{F}_{r}\right) \mathrm{d} \mathbb{P}(g)=\int_{A^{\prime}} \int_{X} f(x) \nu_{r}\left(\eta_{r} \Theta_{r} g, \mathrm{~d} x\right) \mathrm{d} \mathbb{P}(g) .
$$

The left side is equal to

$$
\int_{A^{\prime}} f\left(\chi \mathcal{O}_{r} g\right) \mathrm{d} \mathbb{P}(g)=\int_{\Omega} f(\chi g) 1_{A^{\prime}}\left(\mathcal{O}_{r}^{-1} g\right) \rho_{r}(g) \mathrm{d} \mathbb{P}(g)
$$

by the change of measure (2.4). The random transformation $\mathcal{O}_{r}^{-1}$ on $\Omega$ is $\mathcal{F}_{B^{\mathrm{E}}}$ measurable, as is the function $\rho_{r}$. Consequently, the right-hand side of (4.7) is equal to

$$
\int_{\Omega} \mathbb{E}\left(f \chi \cdot 1_{\mathcal{O}_{r} A^{\prime}} \cdot \rho_{r} \mid \mathcal{F}_{B^{\mathrm{E}}}\right) \mathrm{dP}=\int_{\Omega} \mathbb{E}\left(f \chi \mid \mathcal{F}_{B^{\mathrm{E}}}\right) 1_{\mathcal{O}_{r} A^{\prime}} \cdot \rho_{r} \mathrm{~d} \mathbb{P}=\int_{\Omega} \mathbb{E}\left(f \chi \mid \mathcal{F}_{B}\right) 1_{\mathcal{O}_{r} A^{\prime}} \cdot \rho_{r} \mathrm{~d} \mathbb{P}
$$
since $\mathbb{E}\left(f \chi \mid \mathcal{F}_{B^{\mathrm{E}}}\right)=\mathbb{E}\left(f \chi \mid \mathcal{F}_{B}\right)$ by (4.4). Substituting (4.5), this is equal to

$$
\int_{\Omega}\left(\int_{X} f(x) \nu\left(\eta_{r} \chi g, \mathrm{~d} x\right)\right) 1_{\mathcal{O}_{r} A^{\prime}}(g) \rho_{r}(g) \mathrm{d} \mathbb{P}(g)=\int_{A^{\prime}} \int_{X} f(x) \nu\left(\eta_{r} \chi \mathcal{O}_{r} g, \mathrm{~d} x\right) \mathrm{d} \mathbb{P}(g),
$$

where we transform the measure back to $\mathbb{P}$ via (2.4). 
Claim 4.3 (Strong Markov Property). Supposing that Claim 4.2 holds, if $v \in S^{d-1}$ and $f: X \rightarrow \mathbb{R}$ is measurable, then

$$
\mathbb{E}\left(f \circ \Theta_{R} \mid \mathcal{F}_{R}\right)=\int_{X} f(x) \nu_{R}\left(\eta_{R} \Theta_{R} g, \mathrm{~d} x\right)
$$

on the event $\left\{R\right.$ is a frontier of $\left.\gamma_{v}\right\}$ for $\mathbb{P}$-almost every $g \in \Omega$.

This proof follows the classic proof of the Strong Markov Property [Dur96], where we approximate the random frontiers by deterministic radii. We have not yet worked through the argument in full detail, but there should be no technical complications. With the Strong Markov Property in hand, we are ready to prove Claim 2.14:

Proof of Claim 2.14. Let $v \in S^{d-1}$ and let $B \subseteq C^{2+\alpha}(W$, SPD) be open. Since we are considering frontiers, define

$$
\Gamma=\left\{y \in Y:\|y\|_{C^{2+\alpha}\left(B_{\infty}\right)}+\frac{1}{\lambda\left(B_{\infty}\right)} \leq h\right\} \subseteq Y_{\infty}
$$

for the value of $h$ as in Theorem 2.10. Because of the Hölder condition $\alpha$ on the second derivatives, the Arzelà-Ascoli Theorem [Fol99] implies that $\Gamma$ is compact in $Y_{\infty}$

Let

$$
p=\frac{1}{2} \inf _{y \in \Gamma} \nu_{\infty}(y, B) .
$$

Since $B$ is open, Proposition 4.1.b implies that the function $\nu_{\infty}(\cdot, B)$ is lower semicontinuous. Hence on the compact set $\Gamma$ it attains its minimum $2 p$. By Proposition 4.1.a, this is positive so $p>0$.

By the Strong Markov Property,

$$
\mathbb{P}\left(\Theta_{R}^{-1} B \mid \mathcal{F}_{r}\right)=\nu_{R}\left(\eta_{R} \Theta_{R} g, B\right)
$$

on the event $\left\{R\right.$ is a frontier of $\left.\gamma_{v}\right\}$. This event further implies that $\eta_{R} \Theta_{R} g \in \varphi_{R} \Gamma$.

Following the discussion on the definition of $m_{r}$ in the proof of Proposition 4.1.a, define the continuous map $\alpha_{r}=\varphi_{r}^{-1}: Y_{r} \rightarrow Y_{\infty}$ on the dense subspace $\varphi_{r} \eta_{\infty} K \eta_{r}^{*} Y_{r}^{*}$ of $\hat{Y}_{r}$. 
Claim: There exists $R_{1}$ such that if $r \geq R_{1}$, then $\alpha_{r} \varphi_{r} \Gamma \subseteq \Gamma$.

By applying Proposition 4.1.c to $\epsilon=p$, with probability one, there exists $R_{2}>0$ such that if $R \geq \max \left\{R_{1}, R_{2}\right\}$, then

$$
\nu_{R}\left(\eta_{R} \Theta_{R} g, B\right) \geq \nu_{\infty}\left(\alpha_{R} \eta_{R} \Theta_{R} g, B\right)-p \geq \inf _{y \in \Gamma} \nu_{\infty}(y, B)-p=p
$$




\section{REFERENCES}

[AA09] L.P. Arguin and M. Aizenman. On the structure of quasi-stationary competing particle systems. Ann. Probab, 37(3):1080-1113, 2009.

[AL88] V.I. Arnold and M. Levi. Geometrical methods in the theory of ordinary differential equations. Springer, 1988.

[Ale93] K.S. Alexander. A note on some rates of convergence in first-passage percolation. The Annals of Applied Probability, 3(1):81-90, 1993.

[Ale97] K.S. Alexander. Approximation of subadditive functions and convergence rates in limiting-shape results. The Annals of Probability, 25(1):30-55, 1997.

[ALR87] M. Aizenman, J.L. Lebowitz, and D. Ruelle. Some rigorous results on the Sherrington-Kirkpatrick spin glass model. Communications in mathematical physics, 112(1):3-20, 1987.

[ASS07] M. Aizenman, R. Sims, and S.L. Starr. Mean-Field Spin Glass models from the Cavity-ROSt Perspective. In Prospects in mathematical physics: Young Researchers Symposium of the 14th International Congress on Mathematical Physics, July 25-26, 2003, Lisbon, Portugal, page 1. Amer Mathematical Society, 2007.

[BDJ99] J. Baik, P. Deift, and K. Johansson. On the distribution of the length of the longest increasing subsequence of random permutations. Journal of the American Mathematical Society, 12(4):1119-1178, 1999.

[BKS03] I. Benjamini, G. Kalai, and O. Schramm. First passage percolation has sublinear distance variance. Annals of Probability, pages 1970-1978, 2003.

[Bog98] V.I. Bogachev. Gaussian measures. American Mathematical Society, 1998.

[Boi90] D. Boivin. First passage percolation: the stationary case. Probability Theory and Related Fields, 86(4):491-499, 1990.

[Bol89] E. Bolthausen. A note on the diffusion of directed polymers in a random environment. Communications in Mathematical Physics, 123(4):529-534, 1989.

[BR06] M. Benaïm and R. Rossignol. A modified Poincare inequality and its application to First Passage Percolation. arXiv preprint math/0602496, 2006. 
[BR08] M. Benaïm and R. Rossignol. Exponential concentration for First Passage Percolation through modified Poincaré inequalities. Annales de l'Institut Henri Poincaré, Probabilités et Statistiques, 44(3):544-573, 2008.

[BS10] N.D. Blair-Stahn. First Passage Percolation and Competition Models. arXiv preprint arXiv:1005.0649, 2010.

[CD81] J.T. Cox and R. Durrett. Some limit theorems for percolation processes with necessary and sufficient conditions. The Annals of Probability, 9(4):583-603, 1981.

[CD09] S. Chatterjee and P.S. Dey. Central limit theorem for first-passage percolation time across thin cylinders. arXiv preprint arXiv:0911.5702, 2009.

[CH02] P. Carmona and Y. Hu. On the partition function of a directed polymer in a Gaussian random environment. Probability Theory and Related Fields, 124(3):431-457, 2002.

[Cha08] S. Chatterjee. Chaos, concentration, and multiple valleys. arXiv preprint arXiv:0810.4221, 2008.

[Cha09] S. Chatterjee. Disorder chaos and multiple valleys in spin glasses. Arxiv preprint arXiv:0907.3381, 2009.

[CSY03] F. Comets, T. Shiga, and N. Yoshida. Directed polymers in a random environment: path localization and strong disorder. Bernoulli, pages 705723, 2003.

[CSY04] F. Comets, T. Shiga, and N. Yoshida. Probabilistic analysis of directed polymers in a random environment: a review. Advanced Studies in Pure Mathematics, 39:115-142, 2004.

[Der85] B. Derrida. A generalization of the random energy model which includes correlations between energies. Journal de Physique Lettres, 46(9):401-407, 1985 .

[Der90] B. Derrida. Directed polymers in a random medium. Physica A: Statistical and Theoretical Physics, 163(1):71-84, 1990.

[dH09] F. den Hollander. Random polymers: École d'Été de Probabilités de SaintFlour XXXVII-2007. Springer Verlag, 2009.

[DL81] R. Durrett and T.M. Liggett. The shape of the limit set in Richardson's growth model. The Annals of Probability, 9(2):186-193, 1981. 
[Dur96] R. Durrett. Probability: theory and examples. Duxbury Press Belmont, CA, 1996.

[EA75] S.F. Edwards and P.W. Anderson. Theory of spin glasses. Journal of Physics F: Metal Physics, 5:965-974, 1975.

[Fol99] G.B. Folland. Real Analysis: Modern Techniques and Their Applications. Wiley-Interscience, 1999.

[GH75] D. Geman and J. Horowitz. Random shifts which preserve measure. Proceedings of the American Mathematical Society, 49(1):143-150, 1975.

[Gia07] G. Giacomin. Random polymer models. Imperial College Pr, 2007.

[GT02] F. Guerra and F.L. Toninelli. The thermodynamic limit in mean field spin glass models. Communications in Mathematical Physics, 230(1):71-79, 2002 .

[GT06] G. Giacomin and F.L. Toninelli. Smoothing effect of quenched disorder on polymer depinning transitions. Communications in Mathematical Physics, 266(1):1-16, 2006.

[HH85] D.A. Huse and C.L. Henley. Pinning and roughening of domain walls in Ising systems due to random impurities. Physical review letters, 54(25):2708-2711, 1985.

[HHF85] D.A. Huse, C.L. Henley, and D.S. Fisher. Huse, Henley, and Fisher respond. Physical Review Letters, 55(26):2924-2924, 1985.

[HM95] O. Häggström and R. Meester. Asymptotic shapes for stationary first passage percolation. The Annals of Probability, 23(4):1511-1522, 1995.

[HM07] B. Hambly and J.B. Martin. Heavy tails in last-passage percolation. Probability Theory and Related Fields, 137(1):227-275, 2007.

[HN97] C.D. Howard and C.M. Newman. Euclidean models of first-passage percolation. Probability Theory and Related Fields, 108(2):153-170, 1997.

[HN00] C.D. Howard and C.M. Newman. Geodesics and spanning trees for Euclidean first-passage percolation. arXiv preprint math/0010205, 2000.

[HN01] C.D. Howard and C.M. Newman. Special Invited Paper: Geodesics And Spanning Trees For Euclidean First Passage Percolation. Ann. Probab, 29(2):577-623, 2001. 
[Hof08] C. Hoffman. Geodesics in first passage percolation. Ann. Appl. Probab, 18(5):1944-1969, 2008.

[How00] C.D. Howard. Lower bounds for point-to-point wandering exponents in Euclidean first-passage percolation. Journal of Applied Probability, 37(4):1061-1073, 2000.

[How04] C.D. Howard. Models of first-passage percolation. Probability on Discrete Structures, pages 125-173, 2004.

[HP98] O. Häggström and R. Pemantle. First passage percolation and a model for competing spatial growth. Journal of Applied Probability, 35(3):683-692, 1998.

[HW65] J.M. Hammersley and D.J.A. Welsh. First-passage percolation, subadditive process, stochastic network and generalized renewal theory. Bernoulli, 1713: Bayes, 1763; Laplace, 1813. Anniversary Volume, page 61, 1965.

[IR78] I.A. Ibragimov and J.A. Rozanov. Gaussian random processes. SpringerVerlag New York, 1978.

[IS88] J.Z. Imbrie and T. Spencer. Diffusion of directed polymers in a random environment. Journal of Statistical Physics, 52(3):609-626, 1988.

[Joh00] K. Johansson. Shape fluctuations and random matrices. Communications in mathematical physics, 209(2):437-476, 2000.

[Kes84] H. Kesten. Aspects of first passage percolation. Ecole d'été de Probabilités de St. Flour. Lecture Notes in Math, 1180:125-264, 1984.

[Kes93] H. Kesten. On the speed of convergence in first-passage percolation. The Annals of Applied Probability, 3(2):296-338, 1993.

[Kin68] J.F.C. Kingman. The ergodic theory of subadditive stochastic processes. Journal of the Royal Statistical Society. Series B (Methodological), 30(3):499-510, 1968.

[KN85] M. Kardar and D.R. Nelson. Commensurate-incommensurate transitions with quenched random impurities. Physical review letters, 55(11):11571160, 1985 .

[KPZ86] M. Kardar, G. Parisi, and Y.C. Zhang. Dynamic scaling of growing interfaces. Physical Review Letters, 56(9):889-892, 1986. 
[KS88] J. Krug and H. Spohn. Universality classes for deterministic surface growth. Physical Review A, 38(8):4271-4283, 1988.

[KS91] J. Krug and H. Spohn. Kinetic roughening of growing surfaces. Solids far from equilibrium, pages 479-582, 1991.

[LaG10] T. LaGatta. Continuous Disintegrations of Gaussian Measures. arXiv preprint arXiv:1003.0975, 2010.

[Lee97] J.M. Lee. Riemannian Manifolds: An Introduction to Curvature. Springer, 1997.

[LN96] C. Licea and C.M. Newman. Geodesics in two-dimensional first-passage percolation. The Annals of Probability, 24(1):399-410, 1996.

[LNP96] C. Licea, C.M. Newman, and M.S.T. Piza. Superdiffusivity in first-passage percolation. Probability Theory and Related Fields, 106(4):559-591, 1996.

[LRST03] V.G. Lamburt, E.R. Rozendorn, D.D. Sokoloff, and V.N. Tutubalin. Geodesics with random curvature on Riemannian and pseudo-Riemannian manifolds. Trudy Geom. Sem. Kazan Gos. Univ, 24:99-106, 2003.

[LW10] T. LaGatta and J. Wehr. A Shape Theorem for Riemannian First-Passage Percolation. J. Math. Phys., 51(5), 2010.

[Mar00] J.B. Martin. Linear growth for greedy lattice animals. Rapport de recherche-institut national de recherche en informatique et en automatique, 2000 .

[Mar04] J.B. Martin. Limiting shape for directed percolation models. Annals of probability, 32(4):2908-2937, 2004.

[MPV87] M. Mezard, G. Parisi, and M.A. Virasoro. Spin glass theory and beyond. World Scientific Singapore, 1987.

[New95] C.M. Newman. A surface view of first-passage percolation. In S.D. Chatterji, editor, Proceedings of the International Congress of Mathematicians, volume 2, pages 1017-1023, 1995.

[New97] C.M. Newman. Topics in disordered systems. Birkhäuser, 1997.

[New10] C.M. Newman. personal communication, 2010.

[NP95] C.M. Newman and M.S.T. Piza. Divergence of shape fluctuations in two dimensions. The Annals of Probability, 23(3):977-1005, 1995. 
[Par79] G. Parisi. Infinite number of order parameters for spin-glasses. Physical Review Letters, 43(23):1754-1756, 1979.

[Piz97] M.S.T. Piza. Directed polymers in a random environment: some results on fluctuations. Journal of Statistical Physics, 89(3):581-603, 1997.

[PRT00] G. Parisi and F. Ricci-Tersenghi. On the origin of ultrametricity. Journal of Physics A: Mathematical and General, 33:113-129, 2000.

[Ric73] D. Richardson. Random growth in a tessellation. In Proceedings of the Cambridge Philosophical Society, volume 74, page 515, 1973.

[Rue87] D. Ruelle. A mathematical reformulation of Derrida's REM and GREM. Communications in Mathematical Physics, 108(2):225-239, 1987.

[SK75] D. Sherrington and S. Kirkpatrick. Solvable model of a spin-glass. Physical review letters, 35(26):1792-1796, 1975.

[SZ96] R. Song and X.Y. Zhou. A remark on diffusion of directed polymers in random environments. Journal of Statistical Physics, 85(1):277-289, 1996.

[Tal94] M. Talagrand. On Russo's approximate zero-one law. The Annals of Probability, 22(3):1576-1587, 1994.

[Tal98] M. Talagrand. The Sherrington-Kirkpatrick model: a challenge for mathematicians. Probability Theory and Related Fields, 110(2):109-176, 1998.

[TW94] C.A. Tracy and H. Widom. Level-spacing distributions and the Airy kernel. Communications in Mathematical Physics, 159(1):151-174, 1994.

[VAW90] M.Q. Vahidi-Asl and J.C. Wierman. First-passage percolation on the Voronoi tessellation and Delaunay triangulation. In Random graphs' 8\%: based on proceedings of the 3rd International Seminar on Random Graphs and Probabilistic Methods in Combinatorics, June 27-July 3 1987, Poznań, Poland, page 341. John Wiley \& Sons Inc, 1990.

[VAW92] M.Q. Vahidi-Asl and J.C. Wierman. A shape result for first-passage percolation on the Voronoi tessellation and Delaunay triangulation. In Random graphs, volume 2, pages 247-262. Wiley-Interscience, 1992.

[WA90] J. Wehr and M. Aizenman. Fluctuations of extensive functions of quenched random couplings. Journal of Statistical Physics, 60(3):287-306, 1990.

[Weh97] J. Wehr. On the number of infinite geodesics and ground states in disordered systems. Journal of Statistical Physics, 87(1):439-447, 1997. 
[WW98] J. Wehr and J. Woo. Absence of geodesics in first-passage percolation on a half-plane. Annals of Probability, 26(1):358-367, 1998.

[Zir01] C.L. Zirbel. Lagrangian observations of homogeneous random environments. Advances in Applied Probability, 33(4):810-835, 2001. 


\section{Appendix A \\ A Shape Theorem for Riemannian First-Passage PERCOLATION}

Published in Journal of Mathematical Physics.

Received 17 December 2009; accepted 1 April 2010; published online 3 May 2010. 


\section{AMERICAN INSTITUTE OF PHYSICS \\ Suite 1NO1, 2 Huntington Quadrangle}

Melville, NY 11747-4502 USA

The following transfer agreement must be signed and returned to the Editor's Office before the manuscript can be published. For further information about AIP policies and practices regarding copyright, see http://journals.aip.org/copyright.html or contact the Office of Rights and Permissions, American Institute of Physics, Suite 1NO1, 2 Huntington Quadrangle, Melville, NY 11747-4502 USA; Phone: 516-576-2268; Email: rights@aip.org.

Article Title: A ShapeTheorem for Ripmannian Fivjt.Pajgage Perrol.tion

Names of All Authors: Tom LaGatta and Jan Wehr

Journal: Journal of Mothemetical Physics

\section{TRANSFER OF COPYRIGHT AGREEMENT}

Copyright to the above-listed unpublished and original article submitted by the above author(s), the abstract forming part thereof, and any subsequent errata (collectively, the "Article") is hereby transferred to the American Institute of Physics (AIP) for the full term thereof throughout the world, subject to the Author Rights (as hereinafter defined) and to acceptance of the Article for publication in a journal of AIP. This transfer of copyright includes all material to be published as part of the Article (in any medium), including but not limited to tables, figures, graphs, movies, and other multimedia files. AIP shall have the right to register copyright to the Article in its name as claimant, whether separately or as part of the journal issue or other medium in which the Article is included.

The author(s), and in the case of a Work Made For Hire, as defined in the U.S. Copyright Act, 17 U.S.C. § 101, the employer named below, shall have the following rights (the "Author Rights"):

(1) All proprietary rights other than copyright, such as patent rights

(2) The nonexclusive right, after publication by AIP, to give permission to third parties to republish print versions of the Article or a translation thereof, or excerpts therefrom, without obtaining permission from AIP, provided the AIP-prepared version is not used for this purpose, the Article is not published in another journal, and the third party does not charge a fee. If the AIP version is used, or the third party republishes in a publication or product charging a fee for use, permission from AIP must be obtained.

(3) The right to use all or part of the Article, including the AIP-prepared version without revision or modification, on the author(s)' web home page or employer's website and to make copies of all or part of the Article for the author(s)' and/or the employer's use for lecture or classroom purposes. If a fee is charged for any use, AIP permission must be obtained.

(4) The right to post and update the Article on free-access e-print servers as long as files prepared and/or formatted by AIP or its vendors are not used for that purpose. Any such posting made or updated after acceptance of the Article for publication shall include a link to the online abstract in the AIP journal or to the entry page of the journal. If the author wishes the AIP-prepared version to be used for an online posting other than on the author(s)' or employer's website, AIP permission is required; if permission is granted, AIP will provide the Article as it was published in the journal, and use will be subject to AIP terms and conditions.

(5) If the Article was prepared under a U.S. Government contract, the government shall have the rights under the copyright to the extent required by the contract.

All copies of the Article made under any of the Author Rights shall include notice of the AIP copyright.

By signing this Agreement, the author(s), and in the case of a Work Made For Hire, the employer, jointly and severally represent and warrant that the Article is original with the author(s) and does not infringe any copyright or violate any other right of any third parties, and that the Article has not been published elsewhere, and is not being considered for publication elsewhere in any form, except as provided herein. If each author's signature does not appear below, the signing author(s) represent that they sign this Agreement as authorized agents for and on behalf of all the authors, and that this Agreement and authorization is made on behalf of all the authors. The signing author(s) (and, in the case of a Work Made For Hire, the signing employer) also represent and warrant that they have the full power to enter into this Agreement and fo make the grants contained herein.
Author Signature(s) John Lebifta
Name(s) (print)

If the Article has been prepared as a Work Made For Hire, the transfer should be signed by both the employee (above) and the employer (below):

\section{Employer}

Authorized Signature(s)

\section{U.S. GOVERNMENT EMPLOYEES}

A work prepared by a U.S. Government officer or employee* as part of his or her official duties is not eligible for U.S. copyright. If at least one of the authors is not in this category, that author should sign the transfer Agreement above. If all the authors are in this category, one of the authors should sign below, and indicate his or her affiliation.

\begin{tabular}{l}
\hline Author Signature(s) Name(s) (print) Institution (e.g., Naval Research Laboratory, NIST) \\
*Employees of national laboratories, e.g. Argonne National Laboratory, are not U.S. Government employees.
\end{tabular}




\title{
A SHAPE THEOREM FOR RIEMANNIAN FIRST-PASSAGE PERCOLATION
}

\author{
T. LAGATTA AND J. WEHR
}

\begin{abstract}
Riemannian first-passage percolation (FPP) is a continuum model, with a distance function arising from a random Riemannian metric in $\mathbb{R}^{d}$. Our main result is a shape theorem for this model, which says that large balls under this metric converge to a deterministic shape under rescaling. As a consequence, we show that smooth random Riemannian metrics are geodesically complete with probability one.
\end{abstract}

\section{INTRODUCTION}

1.1. Motivation. We introduce Riemannian first-passage percolation (FPP) as a model of random geometry in the continuum. Our work is motivated by standard FPP, where one builds a random distance function on $\mathbb{Z}^{d}$ from independent, identically-distributed "passage times" over bonds of the lattice. We instead construct our distance function on $\mathbb{R}^{d}$ from a random Riemannian metric. The main result of the paper is a shape theorem for Riemannian FPP: if $B_{t}$ is the random Riemannian ball of radius $t$, then $\frac{1}{t} B_{t}$ tends toward a limiting shape. From this it follows that a random Riemannian metric is geodesically complete with probability one.

Hammersley and Welsh [7] introduced standard FPP in 1965 in order to model fluid flow through porous media. Consider the $d$-dimensional lattice $\mathbb{Z}^{d}$ with $d \geq 2$. Let $\left\{t_{b}\right\}$ be a family of independent, identically distributed, non-negative random variables, indexed by bonds (nearest-neighbor edges) $b$ of the lattice. For any $z, z^{\prime} \in$ 
$\mathbb{Z}^{d}$, define

$$
d\left(z, z^{\prime}\right)=\inf _{\gamma} \sum_{b \in \gamma} t_{b}
$$

where the infimum is taken over all lattice paths $\gamma$ connecting $z$ to $z^{\prime}$. This $d$ is a random distance function on $\mathbb{Z}^{d}$. For a very good introduction to standard FPP, see Howard [8].

Consider the random distance $d\left(0, n \mathrm{e}_{1}\right)$ between the origin and the point $n \mathrm{e}_{1}=$ $(n, 0, \ldots, 0)$. One wishes to study the asymptotic behavior of this quantity as $n \rightarrow \infty$. In [12], Kingman formulated his famous subadditive ergodic theorem in order to prove the basic result of FPP: provided the passage times have finite mean, there exists a non-random constant $\mu_{\mathrm{e}_{1}}$, such that

$$
\lim _{n \rightarrow \infty} \frac{1}{n} d\left(0, n \mathrm{e}_{1}\right)=\mu_{\mathrm{e}_{1}}
$$

almost surely and in $L^{1}$. The same is clearly true for all coordinate axes and, more generally, for each direction $v \in S^{d-1}$, there exists a non-random constant $\mu_{v}$, such that

$$
\lim _{n \rightarrow \infty} \frac{1}{n} d(0, \widetilde{n v})=\mu_{v}
$$

almost surely and in $L^{1}$, where $\widetilde{n v} \in \mathbb{Z}^{d}$ is the nearest lattice point to $n v$. The constant $\mu_{v}$ is non-zero provided that the probability that $t_{b}=0$ is less than the critical percolation probability for $\mathbb{Z}^{d}[8]$.

The shape theorem of Cox and Durrett [2] is a stronger result. Consider

$$
\tilde{B}_{t}=\left\{z \in \mathbb{Z}^{d}: d(0, z) \leq t\right\}
$$

the random ball of radius $t$ in $\mathbb{Z}^{d}$. This is a lattice object, so we "inflate" it to get a continuum one: for $z \in \mathbb{Z}^{d}$, let $C_{z}=[z-1 / 2, z+1 / 2)^{d}$ be the unit cube centered at 
$z$ in $\mathbb{R}^{d}$, and let

$$
B_{t}=\bigcup_{z \in \tilde{B}_{t}} C_{z} .
$$

We define the rescaling $\frac{1}{t} B_{t}$ as the set of all points $x \in \mathbb{R}^{d}$ such that $t x \in B_{t}$. The shape theorem says that there exists a non-random convex, compact set $A$, depending only on the distribution of $t_{b}$, such that $\frac{1}{t} B_{t} \rightarrow A$ : for all $\epsilon>0$, with probability one, there exists a time $T$ such that if $t \geq T$, then

$$
(1-\epsilon) A \subseteq \frac{1}{t} B_{t} \subseteq(1+\epsilon) A
$$

Just as standard FPP is a model of random geometry on the discrete lattice $\mathbb{Z}^{d}$, Riemannian FPP is a model of random geometry in the continuum. We consider a random Riemannian metric $g$ on $\mathbb{R}^{d}$ whose distribution is translation-invariant, has finite-range dependence and satisfies certain moment conditions. By the standard construction in Riemannian geometry, this defines a random distance function $d(x, y)$ in $\mathbb{R}^{d}$. Kingman's theorem can again be applied to prove that for each unit vector $v$, there exists a non-random constant $\mu_{v} \geq 0$ such that $\frac{1}{n} d(0, n v) \rightarrow \mu_{v}$ a.s. and in $L^{1}$. From the positive-definiteness of $g$, we prove Theorem 2.5: $\mu_{v}>0$ for all $v \in S^{d-1}$. Consider the set

$$
A=\left\{x \in \mathbb{R}^{d}:|x| \leq \mu_{x /|x|}^{-1}\right\}
$$

and the Riemannian ball of radius $t$ centered at the origin

$$
B_{t}=\left\{x \in \mathbb{R}^{d}: d(0, x) \leq t\right\}
$$

Theorem 3.1 is the shape theorem for Riemannian FPP, which states that for all $\epsilon>0$, with probability one, there exists a random time $T>0$ such that if $t \geq T$, then

$$
(1-\epsilon) A \subseteq \frac{1}{t} B_{t} \subseteq(1+\epsilon) A
$$


Consequently, we call $A$ the limiting shape of the model.

Theorem 3.4 follows from the shape theorem: with probability one, no curve $\gamma$, parametrized by Riemannian length, reaches infinity in finite time. When the metric is further assumed to be smooth with probability one, then this is geometrically significant: by the Hopf-Rinow theorem [13], this is equivalent to geodesic completeness of the metric.

To prove our results, we need some technical estimates on the distance function $d$, which we obtain in Section 2. To prove these, we discretize the continuum model: to each point $z \in \mathbb{Z}^{d}$, we associate a certain value $X_{z}$ based on the Riemannian metric $g(x)$ over the unit cube $C_{z}=[z-1 / 2, z+1 / 2)^{d}$. By treating $X_{z}$ as defining a dependent FPP model on the lattice, we prove some estimates of the "entropy-energy type" on $X_{z}$, and from these derive the desired estimates on $d$.

1.2. Geometry Background and Notation. Before introducing any probabilistic structure, we introduce some geometric notation. Consider $\mathbb{R}^{d}$ with $d \geq 2$ and the standard Euclidean coordinates. Write

$$
\mathrm{SPD}=\{\text { symmetric, positive-definite } d \times d \text { real matrices }\}
$$

and let $g \in C\left(\mathbb{R}^{d}, \mathrm{SPD}\right)$ be a continuous matrix-valued function on $\mathbb{R}^{d}$ with values in SPD. $g$ defines a Riemannian structure on $\mathbb{R}^{d}$ : for tangent vectors $v, v^{\prime} \in T_{x} \mathbb{R}^{d}$, we consider the inner product $\left\langle v, g(x) v^{\prime}\right\rangle$. For a single vector $v$, we denote by $\|v\|=\sqrt{\langle v, g(x) v\rangle}$ and $|v|=\sqrt{\langle v, v\rangle}$ the Riemannian and Euclidean lengths of $v$, respectively. For a $C^{1}$-curve $\gamma:[a, b] \rightarrow \mathbb{R}^{d}$, we define the Riemannian and Euclidean lengths of $\gamma$ by

$$
R(\gamma)=\int_{a}^{b}\|\dot{\gamma}(t)\| \mathrm{d} t \quad \text { and } \quad L(\gamma)=\int_{a}^{b}|\dot{\gamma}(t)| \mathrm{d} t
$$


respectively. We say that a curve is finite if it has finite Euclidean length; for our model, Theorem 3.4 will imply that finite curves have finite Riemannian length. The Riemannian distance between two points $x$ and $y$ is defined by

$$
d(x, y)=\inf _{\gamma} R(\gamma)
$$

where the infimum is over all $C^{1}$-curves $\gamma$ connecting $x$ to $y$.

For a Riemannian metric $g$, we define the real, positive functions

$\Lambda(x)=$ maximum eigenvalue of $g(x) \quad$ and $\quad \lambda(x)=$ minimum eigenvalue of $g(x)$.

For any $K \subseteq \mathbb{R}^{d}$, define

$$
\Lambda(K)=\sup _{x \in K} \Lambda(x) \quad \text { and } \quad \lambda(K)=\inf _{x \in K} \lambda(x) .
$$

By the continuity and positivity of $g$, if $K$ is bounded then

$$
0<\lambda(K) \leq \Lambda(K)<\infty
$$

For $z \in \mathbb{Z}^{d}$, let $C_{z}=[z-1 / 2, z+1 / 2)^{d}$ be the unit cube centered at $z$. Write

$$
\Lambda_{z}=\Lambda\left(C_{z}\right) \quad \text { and } \quad \lambda_{z}=\lambda\left(C_{z}\right)
$$

1.3. Riemannian FPP. Let $\Omega=C\left(\mathbb{R}^{d}, \mathrm{SPD}\right)$ and let $\mathcal{F}$ be the $\sigma$-algebra generated by cylinder sets. Let $\mathbb{P}$ be a translation-invariant probability measure on $(\Omega, \mathcal{F})$ which has finite-range dependence, and consider a random Riemannian metric $g \in \Omega$ with distribution $\mathbb{P}$. The finite-range dependence means there exists some $R>0$ such that if $|x-y| \geq R$, then $g(x)$ and $g(y)$ are independent. Furthermore, suppose that $\Lambda_{0}$ has a finite moment-generating function. That is,

$$
M(r)=\mathbb{E}\left[\mathrm{e}^{r \Lambda_{0}}\right]<\infty \quad \text { for all } r \in \mathbb{R}
$$


where $\mathbb{E}$ denotes expectation with respect to $\mathbb{P}$. By translation invariance, the family $\left\{\Lambda_{z}\right\}$ is identically distributed, and we refer to its generic element as $\Lambda$; similarly for $\left\{\lambda_{z}\right\}$ and $\lambda$. By Chebyshev's inequality [4], (1.2) implies that $\Lambda$ and $\lambda$ have exponential tail decay:

$$
\mathbb{P}(\lambda>u) \leq \mathbb{P}(\Lambda>u) \leq M(r) \mathrm{e}^{-r u}
$$

for all $u>0$ and $r>0$.

We provide a concrete example.

Example 1.1. Let $c:[0, \infty) \rightarrow \mathbb{R}$ be a compactly-supported covariance function (see [6] for examples). Let $\xi: \mathbb{R}^{d} \rightarrow \mathbb{R}$ be a mean-zero, stationary, istropic Gaussian field with covariance function $c$; that is,

$$
\mathbb{E}[\xi(x)]=0 \quad \text { and } \quad \mathbb{E}[\xi(x) \xi(y)]=c(|x-y|)
$$

for all $x, y \in \mathbb{R}^{d}$. The covariance $c$ must satisfy certain necessary and sufficient conditions [17] for the field $\xi$ to be everywhere continuous with probability one; suppose this is the case.

Let $g: \mathbb{R}^{d} \rightarrow \mathbb{R}$ be the diagonal matrix with entries

$$
g_{i i}(x)=\log \left(1+\mathrm{e}^{\xi(x)}\right)
$$

for $1 \leq i \leq d$. This is continuous and positive, so it suffices to show that the assumption (1.2) is satisfied.

Proposition 1.2. Assumption (1.2) is satisfied for this choice of $g$, and the shape theorem (Theorem 3.1) applies. Let $A$ be the limiting shape, defined in (3.1). The measure $\mathbb{P}$ is isotropic, so Corollary 3.2 implies that $A$ is a Euclidean ball. Furthermore, if the field $\xi$ is $C^{1}$ with probability one, then Corollary 3.5 implies that the metric $g$ is geodesically complete. 
Proof. Assume for simplicity that $c(0)=1$. The Gaussian concentration inequality [1] implies that

$$
\mathbb{P}\left(\Lambda_{0}>u\right)=\mathbb{P}\left(\sup \xi>\log \left(e^{u}-1\right)\right) \leq \exp \left(-\log \left(\mathrm{e}^{u}-1\right)^{2} / 2\right) \leq 2 \mathrm{e}^{-u^{2} / 2} .
$$

The fundamental theorem of calculus and Fubini's theorem imply that

$\mathbb{E} \mathrm{e}^{r \Lambda_{0}}=\mathbb{E}\left(1+\int_{0}^{\Lambda_{0}} r \mathrm{e}^{r u} \mathrm{~d} u\right)=1+\int_{0}^{\infty} r \mathrm{e}^{r u} \mathbb{P}\left(\Lambda_{0}>u\right) \mathrm{d} u \leq 1+2 \int_{0}^{\infty} r \mathrm{e}^{r u} \mathrm{e}^{-u^{2} / 2} \mathrm{~d} u$

which is finite for all $r$. Thus (1.2) is satisfied, and the results of this paper apply to the random Riemannian metric $g$.

The random variables $\lambda_{z}$ and $\Lambda_{z}$ give rise to a dependent FPP model on sites of the lattice $\mathbb{Z}^{d}$. In Section 2.1, we prove some general estimates for dependent FPP, then in Section 2.2 we apply these to estimates on our distance function $d$. Our techniques are based on the energy-entropy methods of mathematical physics, where one shows that an event occurs with extremely low probability over one particular connected set ("high energy"), but sums this over all possible connected sets at the origin ("high entropy"). One adjusts parameters in the problem so that this sum converges, then applies the Borel-Cantelli lemma. A large-deviations estimate like 1.2 is critical: the number of connected sets at the origin grows exponentially in $n$, the size of the sets, so the probabilities must decay exponentially in $n$ for the arguments to hold.

In the standard FPP setting of passage times $t_{b}$ across bonds $b$, Cox and Durrett [2] prove that a necessary and sufficient condition for a shape theorem is that $\mathbb{E} \min \left\{t_{1}, \ldots, t_{2 d}\right\}^{d}<\infty$, where $t_{i}$ are $2 d$ independent copies of $t_{b}$. Thus we believe that our assumption (1.2) is not the most general, and can be replaced by a finite moment estimate on $\Lambda$ instead to prove a more general result.

In probability theory, Kingman's subadditive ergodic theorem [4] is used to prove that stationary, subadditive sequences obey laws of large numbers. If $X_{n, m}$ is a 
non-negative, stationary sequence which satisfies $X_{n, m} \leq X_{n, r}+X_{r, m}$, then $\frac{1}{n} X_{0, n}$ converges almost surely and in $L^{1}$. Furthermore, if the sequence is ergodic, this convergence is to a non-random constant. In our context, the sequence in question is $X_{n, m}=d(n v, m v)$ for a fixed unit vector $v$. The subadditivity condition is exactly the triangle inequality for $d$, so Kingman's theorem implies that for each $v \in S^{d-1}$, there exists a non-random constant $\mu_{v} \geq 0$ such that

$$
\lim _{t \rightarrow \infty} \frac{1}{t} d(0, t v)=\mu_{v}
$$

almost surely and in $L^{1}$. The constants $\mu_{v}$ may depend on the direction $v$, though if the measure $\mathbb{P}$ is isotropic (rotationally-invariant) then $\mu_{v}=\mu$ will be independent of $v$. We show in Theorem 2.5 that $\mu_{v}>0$ for all $v$.

Proposition 1.3. $\mu_{v}$ is a continuous function of $v$.

Proof. This remarkably short proof is due to Kesten [11] (see his Proof of Theorem 1.7 on page 158). First we show that the function $\mu_{v}$ is bounded above as a function of $S^{d-1}$. Write $v=\sum_{1}^{d} v^{i} \mathrm{e}_{i}$, for the standard basis vectors $\mathrm{e}_{i}$ in $\mathbb{R}^{d}$. We use the triangle inequality to bound $d(0, t v)$ by the sum of the distances between successive points $0, t v^{1} \mathrm{e}_{1}, t v^{1} \mathrm{e}_{1}+t v^{2} \mathrm{e}_{2}$ and so on until $t v$. Translation-invariance implies

$$
\mathbb{E} d(0, t v) \leq \mathbb{E} d\left(0, t v^{1} \mathrm{e}_{1}\right)+\cdots+\mathbb{E} d\left(0, t v^{d} \mathrm{e}_{d}\right)
$$

Dividing by $t$ and taking the limit $t \rightarrow \infty$ gives

$$
\mu_{v}=\lim _{t \rightarrow \infty} \frac{1}{t} \mathbb{E} d(0, t v) \leq \sum_{i=1}^{d} \lim _{t \rightarrow \infty} \frac{1}{t} \mathbb{E} d\left(0, t v^{i} \mathrm{e}_{d}\right) .
$$


The terms which equal zero we may ignore; for the non-zero terms, we make the substitution $t^{\prime}=\left|v^{i}\right| t$, so that the right-hand side equals

$$
\sum_{i=1}^{d} \lim _{t^{\prime} \rightarrow \infty} \frac{\left|v^{i}\right|}{t^{\prime}} \mathbb{E} d\left(0, \pm t^{\prime} \mathrm{e}_{i}\right)=\sum_{i=1}^{d}\left|v^{i}\right| \mu_{\mathrm{e}_{i}} \leq d \max \left\{\mu_{\mathrm{e}_{i}}\right\}
$$

as desired.

Now, consider two different unit vectors $v$ and $v^{\prime}$, and write $u=\frac{v-v^{\prime}}{\left|v-v^{\prime}\right|}$. By the same arguments,

$$
\left|\mu_{v}-\mu_{v^{\prime}}\right| \leq \lim _{t \rightarrow \infty} \frac{1}{t} \mathbb{E} d\left(t v, t v^{\prime}\right)=\mu_{u}\left|v-v^{\prime}\right|
$$

This tends to zero as $v^{\prime} \rightarrow v$ since $\mu_{u}$ is bounded above.

\section{Discretization Lemmas}

2.1. Dependent FPP on a Lattice. For a continuous curve $\gamma$, we would like to introduce a discrete analogue $\Gamma$ on the lattice. For example, $z \in \Gamma$ if $\gamma$ meets the cube $C_{z}$. However, this set $\Gamma$ is not connected on $\mathbb{Z}^{d}$; consider the straight line from 0 to $(1,1)$ in $\mathbb{R}^{2}$. We get around this by modifying the familiar graph structure of $\mathbb{Z}^{d}$ to introduce a new lattice, which we call the $*$-lattice. In this section, we prove some estimates for dependent FPP on the $*$-lattice, then in Section 2.2 we apply these estimates to the continuum model.

For $z \in \mathbb{Z}^{d}$, we write $z=\left(z^{1}, \ldots, z^{d}\right)$. We say that $z, z^{\prime} \in \mathbb{Z}^{d}$ are $*$-adjacent if $\max _{1 \leq i \leq d}\left(z-z^{\prime}\right)^{i} \leq 1$. The $*$-lattice is the graph with vertex set $\mathbb{Z}^{d}$, and edge set given by *-adjacency; that is, the usual lattice $\mathbb{Z}^{d}$ along with all the diagonal edges.

We say that a set $\Gamma \subseteq \mathbb{Z}^{d}$ is $*$-connected if for all $z, z^{\prime} \in \Gamma$, there is a path from $z$ to $z^{\prime}$ along the $*$-lattice which remains in the set $\Gamma$. Technically, that there is a finite sequence of $*$-adjacent points beginning with $z$ and ending with $z^{\prime}$, all contained in $\Gamma$.

Let $S_{n}$ be the number of $*$-connected sets which contain the origin. 
Lemma 2.1. There exists $\sigma$ such that $S_{n} \leq \sigma^{n}$. Obviously, $\sigma>1$.

Proof. Clearly, $\log S_{n}$ is a non-negative subadditive sequence:

$$
\log S_{n+m} \leq \log S_{n}+\log S_{m}
$$

By Fekete's lemma [5], there exists a constant $a \geq 0$ such that $\log S_{n} \leq$ an for all $n$. Defining $\sigma=\mathrm{e}^{a}$ proves the result.

Let $X_{z}$ be a stationary, non-negative random field on the $*$-lattice with finite-range dependence, and with a finite moment-generating function

$$
M(r)=\mathbb{E}\left[\mathrm{e}^{r X}\right]<\infty \quad \text { for all } r \in \mathbb{R}
$$

The finite-range dependence means that there is an integer $R \geq 1$ such that if $\left|z-z^{\prime}\right| \geq$ $R$, then $X_{z}$ and $X_{z^{\prime}}$ are independent.

If $\Gamma \subseteq \mathbb{Z}^{d}$ is a collection of lattice points, we write

$$
X(\Gamma)=\sum_{z \in \Gamma} X_{z}
$$

and call this the passage time of $\Gamma$.

The following two lemmas can be thought of as spatial laws of large numbers. The first says that for sufficiently large $n$, if there is a uniform bound on $X(\Gamma) / n$, then there is also a uniform bound on $|\Gamma| / n$. The second lemma reverses the implication, though with different constants.

Lemma 2.2. Suppose $X_{z}$ additionally satisfies

$$
\mathbb{P}(X=0)<\sigma^{-(2 R+1)^{d}} .
$$


For any $A>0$ there is a non-random $B>0$ such that, with probability one, for any sequence $a_{n} \in \mathbb{Z}^{d}$, there exists $N>0$ such that for all $n \geq N$, if $\Gamma$ is a $*$-connected set which contains the point $a_{n}$ and $X(\Gamma) \leq A n$, then $|\Gamma| \leq B n$.

Lemma 2.3. For any $B>0$ there is a non-random $C>0$ such that, with probability one, for any sequence $a_{n} \in \mathbb{Z}^{d}$, there exists $N>0$ such that for all $n \geq N$, if $\Gamma$ is a *-connected set which contains the point $a_{n}$ and $|\Gamma| \leq B n$, then $X(\Gamma) \leq C n$.

Some assumption like (2.2) is necessary for Lemma 2.2. Let $p=\mathbb{P}(X=0)$, and suppose that $p>p_{c}$, the critical probability for site percolation on the $*$-lattice. By percolation theory, with probability one, the set

$$
\Gamma=\left\{z \in \mathbb{Z}^{d}: X_{z}=0\right\}
$$

contains an infinite $*$-connected component. That is, $|\Gamma|=\infty$ but $X(\Gamma)=0$. No such assumption is necessary for Lemma 2.3.

In this paper, our assumptions of non-negativity and (2.2) are stronger than necessary: we apply these lemmas only to the positive fields $\lambda_{z}$ and $\Lambda_{z}$. However, we anticipate these lemmas to be of independent use in future work on Riemannian FPP, where one may consider fields $X_{z}$ which take the value 0 on a cube $C_{z}$ with small but non-zero probability. For example, if $E_{z}$ is the event that $\Lambda_{z} \leq h$ for a sufficiently large value of $h$, one may apply these lemmas to $X_{z}=1_{E_{z}}$, the indicator function of $E_{z}$.

The generality of the sequence $a_{n}$ is needed for Lemma 2.4. In the proof of that result, we fix a point $x \in \mathbb{R}^{d}$, and define the sequence $a_{n}=\widetilde{n x} \in \mathbb{Z}^{d}$ to be the nearest lattice point to $n x$. 
Proof of Lemma 2.2. In what follows, we assume that $\Gamma$ is a $*$-connected set. Fix $A>0$ and the sequence $a_{n}$. Consider the events

$$
E_{n}=\left\{\exists \Gamma \text { such that } a_{n} \in \Gamma, X(\Gamma) \leq A n \text {, and }|\Gamma|>B n\right\}
$$

We claim that we can choose an integer $B$ large enough so that $\mathbb{P}\left(E_{n}\right)$ decays exponentially in $n$. From the Borel-Cantelli lemma it will follow that, with probability one, only finitely many of the events $E_{n}$ occur, which will prove the result.

Let $z_{i}$ be a predetermined enumeration of $\mathbb{Z}^{d}$; for example, a spiral path beginning at $a_{n}$. For any $*$-connected set $\Gamma$, we define $\Gamma^{\prime} \subseteq \Gamma$ by proceeding along the sequence $z_{i}$ and including each point of $\Gamma$ at a distance at least $R$ away from the previous points chosen. In the form of an algorithm:

- Let $i_{1}$ be the first index for which $z_{i_{1}} \in \Gamma$. Let $g_{1}=z_{i_{1}}$.

- Given $\left\{g_{1}, \ldots, g_{j-1}\right\}$, let $i_{j}$ be the first index for which $z_{i_{j}} \in \Gamma$ and so that $\left|z_{i_{j}}-g_{j^{\prime}}\right|>R$ for $1 \leq j^{\prime}<j$. Let $g_{j}=z_{i_{j}}$.

Let $\Gamma^{\prime}=\left\{g_{1}, g_{2}, \ldots\right\}$. If $\Gamma$ is finite, then so is $\Gamma^{\prime}$. Let

$$
B(z, R)=\left\{z^{\prime} \in \mathbb{Z}^{d}:\left|z-z^{\prime}\right| \leq R\right\}
$$

be the Euclidean ball of radius $R$ centered at $z$ in $Z^{d}$. Note that $|B(z, R)| \leq(2 R+1)^{d}$. To avoid this cumbersome factor $(2 R+1)^{d}$ which appears frequently, for the remainder of this proof we write

$$
K=(2 R+1)^{d}
$$

By construction, the set $\Gamma$ is covered by taking balls around every point in $\Gamma^{\prime}$ :

$$
\Gamma \subseteq \bigcup_{z \in \Gamma^{\prime}} B(z, R)
$$


Let $\Gamma$ be a $*$-connected set described in the event $E_{n}$, so that

$$
B n<|\Gamma| \leq \sum_{z \in \Gamma^{\prime}}|B(z, R)| \leq\left|\Gamma^{\prime}\right| K
$$

Thus, $\left|\Gamma^{\prime}\right|>B n / K$.

We may assume that $\Gamma$ consists only of the first $B n$ points it meets of the sequence $z_{i}$; the non-negativity of $X_{z}$ implies that the passage time $X(\Gamma)$ is still at most $A n$. Similarly, we assume $\left|\Gamma^{\prime}\right|=\lfloor B n / K\rfloor$, the integer part of $B n / K$. Furthermore, since $\Gamma^{\prime} \subseteq \Gamma$

$$
X\left(\Gamma^{\prime}\right) \leq X(\Gamma) \leq A n
$$

Thus the probability $\mathbb{P}\left(E_{n}\right)$ is bounded by

$$
\mathbb{P}\left(\exists \Gamma \text { s.t. } a_{n} \in \Gamma, X\left(\Gamma^{\prime}\right) \leq A n, \text { and }|\Gamma|=B n\right) \leq \sum_{\Gamma} \mathbb{P}\left(X\left(\Gamma^{\prime}\right) \leq A n\right),
$$

where the outer sum is taken over all *-connected sets $\Gamma$ for which $a_{n} \in \Gamma$ and $|\Gamma|=B n$. Lemma 2.1 implies that the number of such sets is bounded by $\sigma^{B n}$.

The family of random variables $\left\{X_{z}\right\}_{\Gamma^{\prime}}$ is independent, since the points $z \in \Gamma^{\prime}$ are separated by distances at least $R$. Let $\left\{X_{i}\right\}$ be $\lceil B n / K\rceil$ independent copies of $X$. The exponential Chebyshev inequality [3] implies that the right-hand side of (2.3) is bounded above by

$$
\sigma^{B n} \mathbb{P}\left(\sum_{i=1}^{\lceil B n / K\rceil} X_{i} \leq A n\right) \leq \sigma^{B n} \mathrm{e}^{r A n} \mathbb{E}\left(\mathrm{e}^{-r \sum X_{i}}\right)
$$

for any $r>0$. Again by independence, if we write $M(-r)=\mathbb{E}^{-r X}$, this is equal to

$$
\sigma^{B n} \mathrm{e}^{r A n}\left(\mathbb{E} \mathrm{e}^{-r X}\right)^{\lceil B n / K\rceil} \leq \sigma^{B n} \mathrm{e}^{r A n} M(-r)^{B n / K}=\left(\sigma \mathrm{e}^{r A / B} M(-r)^{1 / K}\right)^{B n}
$$

By the bounded convergence theorem, as $r$ tends to infinity, $M(-r) \rightarrow \mathbb{P}(X=0)$, which is strictly less than $\sigma^{-K}$ by assumption (2.2). Let $r$ be large enough so that 
$M(-r)<\sigma^{-K}$. Write $p=\sigma M(-r)^{1 / K}<1$, so that

$$
\mathbb{P}\left(E_{n}\right) \leq\left(p \mathrm{e}^{r A / B}\right)^{B n}
$$

Choose the number $B$ to satisfy

$$
r A / \log (1 / p)<B<r A / \log ((1+p) / 2 p)
$$

This implies

$$
\frac{1+p}{2}<p \mathrm{e}^{r A / B}<1
$$

The left inequality will be used later in the proof of Thereom 2.5; the right inequality implies that

$$
\sum \mathbb{P}\left(E_{n}\right) \leq \sum\left(p \mathrm{e}^{r A / B}\right)^{B n}<\infty
$$

so by the Borel-Cantelli lemma, with probability one, only finitely many of the events $E_{n}$ hold.

Proof of Lemma 2.3. In what follows, we assume that $\Gamma$ is a $*$-connected set. Fix an integer $B>0$ and the sequence $a_{n}$. Consider the events

$$
E_{n}=\left\{\exists \Gamma \text { such that } a_{n} \in \Gamma, X(\Gamma)>C n \text {, and }|\Gamma| \leq B n\right\}
$$

We claim that we can choose $C$ large enough so that $\mathbb{P}\left(E_{n}\right)$ decays exponentially in $n$. From the Borel-Cantelli lemma, with probability one, it will follow that only finitely many of the events $E_{n}$ occur, which will prove the result.

As in the proof of Lemma 2.2, we consider passage times of subsets of $\Gamma$ to exploit independence. Unlike in that proof, it does not suffice to consider just one subset $\Gamma^{\prime}$. Instead, we partition the set into $k$ disjoint subsets $\Gamma_{1}, \ldots, \Gamma_{k}$, and consider the passage times of each. 
There are $k=R^{d}$ points in the cube $\{0, \ldots, R-1\}^{d}$; order them $z_{1}, \ldots, z_{k}$. We can partition the lattice $\mathbb{Z}^{d}$ into $k$ subsets by considering $R$-translations of these points. We write $z=\left(z^{1}, \ldots, z^{d}\right)$ for all $z \in \mathbb{Z}^{d}$. For any $*$-connected set $\Gamma$, we partition it into $k$ subsets by defining

$$
\Gamma_{j}=\left\{z \in \Gamma: R \text { divides } z^{i}-z_{j}^{i} \text { for all } i=1, \ldots, d\right\}
$$

for $j=1, \ldots, k$. Since points in $\Gamma_{j}$ are separated by distance at least $R$, for each $j$ the family of random variables $\left\{X_{z}\right\}_{\Gamma_{j}}$ is independent.

Let $\Gamma$ be a set described in the event $E_{n}$. We may assume that $|\Gamma|=B n$, since if it is less, the inclusion of additional points will only increase the passage time calculation. Since

$$
X(\Gamma)=X\left(\Gamma_{1}\right)+\cdots+X\left(\Gamma_{k}\right)
$$

if $X(\Gamma)>C n$, then $X\left(\Gamma_{j}\right)>C n / k$ for some $j$. Furthermore, $\left|\Gamma_{j}\right| \leq|\Gamma| \leq B n$ for each $j$.

As in Lemma 2.2 , the probability $\mathbb{P}\left(E_{n}\right)$ is bounded above by

$$
\sum_{\Gamma} \mathbb{P}\left(\exists j \in\{1, \ldots, k\} \text { s.t. } X\left(\Gamma_{j}\right)>C n / k\right)
$$

where again the sum is over *-connected sets $\Gamma$ for which $a_{n} \in \Gamma$ and $|\Gamma|=B n$. For each $j$, the family of random variables $\left\{X_{i}\right\}_{\Gamma_{j}}$ is independent. Let $\left\{X_{i}\right\}$ be $B n$ independent copies of $X$. The exponential Chebyshev inequality [3] implies that the right-hand side of (2.5) is bounded above by

$$
k \sigma^{B n} \mathbb{P}\left(\sum_{i=1}^{B n} X_{i}>C n / k\right) \leq k \sigma^{B n} \mathrm{e}^{-C n / k} \mathbb{E}\left(\mathrm{e}^{\sum X_{i}}\right)
$$


where we have added extra independent copies of $X_{i}$ to make the number of terms exactly $B n$. If we write $M=M(1)=\mathbb{E} \mathrm{e}^{X}$, then this is equal to

$$
k \sigma^{B n} \mathrm{e}^{-C n / k} M^{B n}=k\left(\sigma^{B} \mathrm{e}^{-C / k} M^{B}\right)^{n} .
$$

We choose $C \gg 1$ so that $\sigma^{B} \mathrm{e}^{-C / k} M^{B}<1$. Thus

$$
\sum \mathbb{P}\left(E_{n}\right) \leq k \sum\left(\sigma^{B} \mathrm{e}^{-C / k} M^{B}\right)^{n}<\infty
$$

so by the Borel-Cantelli lemma, with probability one only finitely many of the events $E_{n}$ hold.

2.2. Applications to Continuum Model. In this section, we apply the estimates from the previous section to the continuum model. Lemma 2.4 says that at large scales, the Riemannian distance function between two points is bounded by a uniform constant $K$ times the Euclidean distance between them. Theorem 2.5 says that $\mu_{v}$ is positive for all $v$.

The cubes $C_{z}$ form a partition of $\mathbb{R}^{d}$. For $x \in \mathbb{R}^{d}$, let $\tilde{x}$ be the unique point on the lattice such that $x \in C_{\tilde{x}}$. The metric $g$ induces two notions of "passage time" over a discrete set $\Gamma$ :

$$
\Lambda(\tilde{\gamma})=\sum_{z \in \tilde{\gamma}} \Lambda_{z} \quad \text { and } \quad \lambda(\tilde{\gamma})=\sum_{z \in \tilde{\gamma}} \lambda_{z}
$$

Lemma 2.4. There exists a non-random $K>0$ such that, with probability one, for all $x \in \mathbb{R}^{d}$ and $\rho>0$, there exists $T(x)>0$ such that if $t \geq T$ and $|x-y| \leq \rho$, then

$$
d(t x, t y) \leq K t \rho
$$

Proof. By rescaling $x$ and $y$, it suffices to prove the lemma with $\rho=1$.

Apply Lemma 2.3 with $B=2 d$ and $X_{z}=\Lambda_{z}$. Thus there exists a non-random $C>0$ such that, with probability one, for any sequence $a_{n} \in \mathbb{Z}^{d}$, there exists $N>0$ 
such that for all $n \geq N$, if $\Gamma$ is a finite $*$-connected set which contains the point $a_{n}$ and $|\Gamma| \leq 2 d n$, then $\Lambda(\Gamma) \leq C n$.

Let $K=C \sqrt{d}$, and suppose that with positive probability, there is some $x \in \mathbb{R}^{d}$ such that for any $n>0$, there exists $y$ (depending on $n$ ) such that $|x-y| \leq 1$ but $d(n x, n y)>K n$. We will show that this leads to a contradiction. Let $N$ be as in Lemma 2.3 applied with the sequence $a_{n}=\widetilde{n x}$.

Suppose that $n \geq N$. Let $\gamma$ be the straight-line segment from $n x$ to $n y$. Let

$$
\Gamma=\left\{z \in \mathbb{Z}^{d}: \gamma \cap C_{z} \neq \emptyset\right\}
$$

index the cubes $C_{z}$ which $\gamma$ meets. Note that $\widetilde{n x} \in \Gamma$. Clearly,

$$
|\Gamma| \leq 2 d|n x-n y| \leq 2 d n
$$

since $|x-y| \leq 1$. By Lemma 2.3,

$$
\Lambda(\Gamma) \leq C n
$$

The distance between $n x$ and $n y$ is a lower bound for the Riemannian length of $\gamma$ :

$$
d(n x, n y) \leq R(\gamma)
$$

Furthermore, since $\gamma$ is a line segment, the Euclidean length of $\gamma$ in each cube is at most $\sqrt{d}$. Thus we can estimate the Riemannian length of $\gamma$ by summing $\Lambda_{z}$ over $\Gamma$ :

$$
d(n x, n y) \leq R(\gamma)=\sum_{z \in \Gamma} R\left(\gamma \cap C_{z}\right) \leq \sum_{z \in \Gamma} \Lambda_{z} \sqrt{d}=\Lambda(\Gamma) \sqrt{d} \leq C n \sqrt{d}=K n
$$

since $K=C \sqrt{d}$. This contradicts the assumption that $d(n x, n y)>K n$.

Theorem 2.5. The constants $\mu_{v}$ are all positive.

Proof. Suppose $\mu=\mu_{v}=0$ for some unit vector $v$. 
Let $\epsilon>0$, and apply Lemma 2.2 with the constant sequence $a_{n} \equiv 0$ to $A=4 \epsilon$ and $X_{z}=\lambda_{z}$. Thus there exists a non-random $B>0$ such that, with probability one, there exists $N_{1}>0$ such that for $n \geq N_{1}$, if $\Gamma$ is a $*$-connected set which contains the origin and $\lambda(\Gamma) \leq 4 \epsilon n$, then $|\Gamma| \leq B n$.

By Kingman's subadditive ergodic theorem, with probability one there exists $N_{2}>$ 0 such that if $n \geq N_{2}$, then

$$
d(0, n v) \leq \epsilon n / 2,
$$

since we assumed that $\mu_{v}=0$.

Let $N=\max \left\{N_{1}, N_{2}\right\}$, and suppose $n \geq N$. A priori, the distance $d(0, n v)$ need not be realized as the Riemannian length of a curve, so let $\gamma$ be a $C^{1}$-curve from 0 to $n v$ with

$$
R(\gamma) \leq d(0, n v)+\epsilon n / 2 \leq \epsilon n,
$$

where the second inequality follows from (2.7).

Define the discrete set

$$
\Gamma=\left\{z \in \mathbb{Z}^{d}: L\left(\gamma \cap C_{z}\right) \geq 1 / 4\right\} ;
$$

that is, $z \in \Gamma$ provided the Euclidean length of $\gamma$ in the cube $C_{z}$ is at least $1 / 4$.

We claim that $\Gamma$ is $*$-connected. Suppose not, so that the continuum set $W=$ $\bigcup_{z \in \Gamma} C_{z}$ has at least two components, separated by Euclidean distance at least 1 . Let $W^{\prime}$ be the $1 / 4$-neighborhood around $W$, so that the components of $W^{\prime}$ are separated by Euclidean distance at least 1/2. By definition of $\Gamma$, the curve $\gamma$ meets each component of $W^{\prime}$, but not the complement $\mathbb{R}^{d} \backslash W^{\prime}$. Since $\gamma$ is continuous, this is a contradiction; hence, $\Gamma$ is $*$-connected. 
In each cube $C_{z}$, we can estimate the Riemannian length of $\gamma$ using $\lambda_{z}$ :

$$
L\left(\gamma \cap C_{z}\right) \lambda_{z} \leq R\left(\gamma \cap C_{z}\right)
$$

where $L$ denotes Euclidean length. Furthermore, by summing $\lambda_{z}$ over the points of $\Gamma$, we get a lower bound for $R(\gamma)$ :

$$
\frac{1}{4} \lambda(\Gamma) \leq \sum_{z \in \Gamma} L\left(\gamma \cap C_{z}\right) \lambda_{z} \leq \sum_{z \in \Gamma} R\left(\gamma \cap C_{z}\right) \leq R(\gamma) \leq \epsilon n
$$

Clearly, $0 \in \Gamma$, so Lemma 2.2 implies that $|\Gamma| \leq B n$.

In the proof of that lemma, we chose $B$ so that

$$
B \leq \frac{r}{\log ((1+p) / 2 p)} A
$$

for positive constants $r$ and $p<1$ not depending on $A$; see (2.4). Since $A=4 \epsilon$, if we write $B^{\prime}=4 r / \log ((1+p) / 2 p)$, then

$$
|\Gamma| \leq B^{\prime} \epsilon n
$$

Let $z$ be a lattice point in $\Gamma$ which minimizes the distance $|n v-z|$. Clearly, $|z| \geq n / 2$. Since $\Gamma$ is $*$-connected and $z \in \Gamma$,

$$
|\Gamma| \geq n / 2 \sqrt{d}
$$

For small $\epsilon$, this contradicts (2.9), so $\mu$ must be positive.

\section{The Shape Theorem and Consequences}

Define the function

$$
\mu(x)= \begin{cases}\mu_{x /|x|}|x|, & x \neq 0 \\ 0, & x=0\end{cases}
$$


Proposition 1.3 and Theorem 2.5 imply that $\mu$ is continuous and, for $x \neq 0$, strictly positive. It follows from the triangle inequality for the distance function that $\mu(x)$ is a norm on $\mathbb{R}^{d}$. Consider the unit ball in this norm,

$$
A=\{x: \mu(x) \leq 1\}=\left\{x:|x| \leq \mu_{x /|x|}^{-1}\right\}
$$

as well as the random Riemannian ball of radius $t$ centered at the origin,

$$
B_{t}=\{x: d(0, x) \leq t\}
$$

Theorem 3.1 (Shape Theorem). For all $\epsilon>0$, with probability one, there exists $T$ such that if $t \geq T$, then

$$
(1-\epsilon) A \subseteq \frac{1}{t} B_{t} \subseteq(1+\epsilon) A
$$

The set $A$ is called the limiting shape of the random Riemannian metric $g$. The shape theorem for lattice first-passage percolation was proved by Cox and Durrett [2]; our proof is modeled on the arguments in Durrett [3].

Proof. It suffices to prove the theorem for $\epsilon \in(0,1)$. Let

$$
\delta<\min \left\{\frac{1}{K}, 1-\frac{1+\epsilon^{2}}{1+\epsilon}\right\},
$$

where $K$ is as in Lemma 2.4. Let $B^{\mathrm{E}}(x, r)$ denote the Euclidean ball of radius $r$ centered at $x$.

We will show that with probability one, there exists $T>0$ such that if $t \geq T$, then $(1-\epsilon) A \subseteq \frac{1}{t} B_{t}$. To do this, we will first prove that for every $x$, there is a random $T(x)$ such that if $t \geq T$, then the small Euclidean ball $B^{\mathrm{E}}\left(x, \delta \epsilon^{2}\right)$ is contained in $\frac{1}{t} B_{t}$. Since the set $(1-\epsilon) A$ is compact, it can be covered by finitely many balls $B^{\mathrm{E}}\left(x_{i}, \delta \epsilon^{2}\right)$. Letting $T=\max \left\{T\left(x_{i}\right)\right\}$ will prove the result. 
Fix $x \in(1-\epsilon) A$. We claim that with probability one, there exists $T(x)$ such that if $t \geq T$ and $|x-y| \leq \delta \epsilon^{2}$, then $d(0, t y) \leq t$, hence $B^{\mathrm{E}}(x, \delta \epsilon) \subseteq \frac{1}{t} B_{t}$ for $t \geq T$. By the triangle inequality,

$$
d(0, t y) \leq d(0, t x)+d(t x, t y)
$$

The first term is controlled by Kingman's theorem: with probability one, there exists $T_{1}(x)$ such that if $t \geq T_{1}$, then

$$
d(0, t x) \leq(1+\epsilon) t \mu(x) \leq\left(1-\epsilon^{2}\right) t
$$

since $\mu(x) \leq(1-\epsilon)$.

The second term is controlled by Lemma 2.4 applied to this $x$ and $\rho=\delta \epsilon^{2}$. With probability one, there exists $T_{2}(x)$ such that for all $t \geq T_{2}$ and $y$ with $|x-y| \leq \delta \epsilon^{2}$, then

$$
d(t x, t y) \leq K t \delta \epsilon^{2}<\epsilon^{2} t
$$

since $\delta<1 / K$.

Let $T(x)=\max \left\{T_{1}, T_{2}\right\}$. If $t \geq T(x)$, then for all $y \in B^{\mathrm{E}}\left(x, \delta \epsilon^{2}\right)$,

$$
d(0, t y) \leq\left(1-\epsilon^{2}\right) t+\epsilon^{2} t=t
$$

implying that $B^{\mathrm{E}}\left(x, \delta \epsilon^{2}\right) \subseteq \frac{1}{t} B_{t}$ for all $t \geq T(x)$. Let $x_{i}$ be finitely many points such that $\bigcup B^{\mathrm{E}}\left(x_{i}, \delta \epsilon^{2}\right)$ covers $(1-\epsilon) A$, and let $T=\max _{i} T\left(x_{i}\right)$. Then for all $t \geq T$,

$$
(1-\epsilon) A \subseteq \bigcup_{i} B^{\mathrm{E}}\left(x_{i}, \delta \epsilon^{2}\right) \subseteq \frac{1}{t} B_{t}
$$

completing the lower half of the shape theorem.

Now we prove the upper half. For any $x$, let $T_{2}(x)$ be as above, so that if $t \geq T_{2}$ and $|x-y|<\delta \epsilon^{2}$, then $d(t x, t y) \leq \epsilon^{2} t$. By Kingman's theorem, with probability one, 
there exists $T_{3}(x)$ such that if $t \geq T_{3}$, then

$$
d(0, t x) \geq(1-\delta) t \mu(x) .
$$

Choose finitely many $x_{i} \in 2 A \backslash(1+\epsilon) A$ such that the closure of $2 A \backslash(1+\epsilon) A$ is covered by $\bigcup B^{\mathrm{E}}\left(x_{i}, \delta \epsilon^{2}\right)$. Belonging to $2 A \backslash(1+\epsilon) A$ implies that $\mu\left(x_{i}\right)>(1+\epsilon)$. Let $T=\max _{i}\left\{T_{2}\left(x_{i}\right), T_{3}\left(x_{i}\right)\right\}$, and let $t \geq T$. Then if $y \in B^{\mathrm{E}}\left(x_{i}, \delta \epsilon^{2}\right)$,

$$
\begin{aligned}
d(0, t y) & \geq d\left(0, t x_{i}\right)-d\left(t x_{i}, t y\right) \\
& \geq(1-\delta) t \mu\left(x_{i}\right)-\epsilon^{2} t \\
& \geq(1-\delta)(1+\epsilon) t-\epsilon^{2} t \\
& >\left(1+\epsilon^{2}\right) t-\epsilon^{2} t=t
\end{aligned}
$$

where the final inequality follows from the assumption (3.3) on $\delta$. Thus $\bigcup B^{\mathrm{E}}\left(x_{i}, \delta \epsilon^{2}\right) \subseteq$ $\frac{1}{t} B_{t}^{c}$ so

$$
2 A \backslash(1+\epsilon) A \subseteq \bigcup B^{\mathrm{E}}\left(x_{i}, \delta \epsilon^{2}\right) \subseteq \frac{1}{t} B_{t}^{c} .
$$

The set $\frac{1}{t} B_{t}$ is connected and contains the origin, hence $\frac{1}{t} B_{t} \subseteq(1+\epsilon) A$ as desired.

Corollary 3.2. If the measure $\mathbb{P}$ is isotropic (rotationally-invariant), then the limiting shape $A$ is the Euclidean ball of radius $\mu^{-1}$.

A simple consequence of Lemma 2.4 is that the convergence (1.3) given by Kingman's theorem is uniform:

Proposition 3.3. For all $\epsilon>0$, with probability one, there exists $T>0$ such that if $t \geq T$, then for all $v \in S^{d-1}$,

$$
\left|\frac{1}{t} d(0, t v)-\mu_{v}\right| \leq \epsilon
$$


Proof. Suppose not. Then with positive probability, there exist $\epsilon>0, t_{n} \rightarrow \infty$ and $v_{n} \in S^{d-1}$ such that

$$
\left|\frac{1}{t_{n}} d\left(0, t_{n} v_{n}\right)-\mu_{v_{n}}\right|>\epsilon,
$$

for all $n$. By compactness of the sphere, a subsequence of $v_{n}$ converges to some $v \in S^{d-1}$; assume without loss of generality that $v_{n} \rightarrow v$. By the triangle inequality,

$$
\epsilon<\left|\frac{1}{t_{n}} d\left(0, t_{n} v_{n}\right)-\frac{1}{t_{n}} d\left(0, t_{n} v\right)\right|+\left|\frac{1}{t_{n}} d\left(0, t_{n} v\right)-\mu_{v}\right|+\left|\mu_{v_{n}}-\mu_{v}\right| .
$$

The second and third terms tend to zero a.s. as $n \rightarrow \infty$ by Kingman's theorem and Proposition 1.3, respectively. By the triangle inequality, the first term is bounded by $\frac{1}{t_{n}} d\left(t_{n} v, t_{n} v_{n}\right)$. Let $\rho=\epsilon / 2 K$, where $K$ is as in Lemma 2.4. Since for large $n$, $\left|v-v_{n}\right| \leq \rho$, we can apply that lemma with $x=v$ to get

$$
\frac{1}{t_{n}} d\left(t_{n} v, t_{n} v_{n}\right) \leq K \rho=\epsilon / 2,
$$

for large $n$ almost surely. This contradicts (3.4).

Theorem 3.4. With probability one, if $\gamma$ is a $C^{1}$-curve parametrized by Riemannian length, then $|\gamma(t)|<\infty$ for all $t \geq 0$.

Proof. It suffices to consider curves starting from the origin. Suppose that with positive probability, there exists a smooth curve $\gamma$ parametrized by Riemannian length which starts from the origin and for which

$$
\lim _{t \rightarrow T}|\gamma(t)|=\infty
$$

for some finite $T$. Since $\gamma$ is parametrized by Riemannian length, for all $t \geq 0$,

$$
t \geq d(0, \gamma(t))
$$


Proposition 3.3 and (3.5) imply that

$$
0=\lim _{t \rightarrow T} \frac{t}{|\gamma(t)|} \geq \lim _{t \rightarrow T} \frac{d(0, \gamma(t))}{|\gamma(t)|} \geq \min _{v} \mu_{v}>0
$$

a contradiction.

We can refine this result if we impose an additional smoothness constraint on the metric. If $g$ is a $C^{2}$-smooth Riemannian metric, i.e. $g \in C^{2}\left(\mathbb{R}^{d}\right.$, SPD), then we can use the calculus of variations to derive the Euler-Lagrange equations for the functional $R$. These are called the geodesic equations [13] for the Riemannian metric $g$, and solutions to this system are called geodesics. The geodesic equations form a secondorder system with locally-Lipschitz coefficients, so a geodesic is uniquely determined by its starting point and velocity. We call a geodesic $\gamma$ length-minimizing if for all $x, y \in \gamma$, the distance $d(x, y)$ is realized as the Riemannian length of the part of the curve $\gamma$ which connects the two points. Not all geodesics are length-minimizing; for example, on the sphere, the geodesics are great circles, which do not minimize length past antipodal points.

A metric is said to be geodesically complete if for all $x \in \mathbb{R}^{d}$ and $v \in T_{x} \mathbb{R}^{d}$, the unique geodesic $\gamma$ at $x$ in direction $v$ can be continued for all time. Part of the Hopf-Rinow theorem [13] of Riemannian geometry is that geodesic completeness is equivalent to the condition stated in Theorem 3.4 for our random metric $g$. A further corollary [13] is that distances are always realized by geodesics. Summarizing, we have

Corollary 3.5. Suppose that, in addition to the assumptions of this paper, $g$ is a $C^{2}$-smooth random Riemannian metric. Then, with probability one, $g$ is geodesically complete. Consequently, for all $x, y \in \mathbb{R}^{d}$, there is a finite length-minimizing geodesic $\gamma$ connecting $x$ to $y$ such that

$$
d(x, y)=R(\gamma)
$$


We alert the reader to a different meaning of the word "geodesic," used often in the first-passage percolation literature. The term is used there to denote a globally length-minimizing path. This is very different from the standard meaning of the word in differential geometry: as described above, geodesics are the curves which locally minimize length, but not necessarily globally. We adhere to this meaning in the present paper.

The existence of two-sided minimizing paths is an open question for all FPP models. For two-dimensional standard FPP, Licea, Newman and Piza [14, 15, 16] have a number of results in this direction. Their work relies on certain curvature assumptions about the limiting shape, which have not been verified for models of independent FPP. Howard and Newman's [9, 10] model of Euclidean FPP, on the other hand, is rotationally invariant. Consequently, the limiting shape is a Euclidean ball, and they prove many results not available in the lattice setting. See the excellent survey [8] for more details. By Corollary 3.2, the limiting shape for isotropic Riemannian FPP is a Euclidean ball. Our hope is for this setting to be a fertile ground for adapting the results referenced in this paragraph.

Remark added after publication: The moment assumptions (1.2) and (2.1) can be slightly weakened to:

$$
M(r)=\mathbb{E}\left[\mathrm{e}^{r \Lambda_{0}}\right]<\infty \quad \text { for all } r \leq a
$$

for some $a>0$. The only modification to the paper is in the proof of Lemma 2.2, where one uses $M=M(a)$ instead of $M=M(1)$. We leave the details to the reader.

\section{REFERENCES}

[1] R.J. Adler and J.E. Taylor. Random Fields and Geometry. Springer New York, 2007.

[2] J.T. Cox and R. Durrett. Some limit theorems for percolation processes with necessary and sufficient conditions. The Annals of Probability, 9(4):583-603, 1981. 
[3] R. Durrett. Lecture notes on particle systems and percolation. The Wadsworth \& Brooks/Cole Statistics/Probability Series, Pacific Grove, CA, 1988.

[4] R. Durrett. Probability: theory and examples. Duxbury Press Belmont, CA, 1996.

[5] M. Fekete. Über die Verteilung der Wurzeln bei gewissen algebraischen Gleichungen mit ganzzahligen Koeffizienten. Mathematische Zeitschrift, 17(1):228-249, 1923.

[6] T. Gneiting. Compactly supported correlation functions. Journal of Multivariate Analysis, 83(2):493-508, 2002.

[7] JM Hammersley and DJA Welsh. First-passage percolation, sub-additive process, stochastic network and generalized renewal theory. Bernoulli, 1713: Bayes, 1763; Laplace, 1813. Anniversary Volume, page 61, 1965.

[8] C.D. Howard. Models of first-passage percolation. Probability on Discrete Structures, pages $125-173,2004$.

[9] C.D. Howard and C.M. Newman. Euclidean models of first-passage percolation. Probability Theory and Related Fields, 108(2):153-170, 1997.

[10] C.D. Howard and C.M. Newman. Special Invited Paper: Geodesics And Spanning Trees For Euclidean First Passage Percolation. Ann. Probab, 29(2):577-623, 2001.

[11] H. Kesten. Aspects of first passage percolation. Ecole d'été de Probabilités de St. Flour. Lecture Notes in Math, 1180:125-264, 1984.

[12] JFC Kingman. The ergodic theory of subadditive stochastic processes. Journal of the Royal Statistical Society. Series B (Methodological), 30(3):499-510, 1968.

[13] J.M. Lee. Riemannian Manifolds: An Introduction to Curvature. Springer, 1997.

[14] C. Licea and C.M. Newman. Geodesics in two-dimensional first-passage percolation. The Annals of Probability, pages 399-410, 1996.

[15] C. Licea, CM Newman, and MST Piza. Superdiffusivity in first-passage percolation. Probability Theory and Related Fields, 106(4):559-591, 1996.

[16] C.M. Newman. Topics in disordered systems. Birkhäuser, 1997.

[17] M. Talagrand. Regularity of Gaussian processes. Acta Mathematica, 159(1):99-149, 1987. 


\section{Appendix B Continuous Disintegrations of Gaussian MEASURES}

Submitted to Journal of Theoretical Probability.

Received 8 April 2010. 


\title{
CONTINUOUS DISINTEGRATIONS OF GAUSSIAN MEASURES
}

\author{
T. LAGATTA
}

\begin{abstract}
A disintegration (or regular conditional probability) is a way to condition a probability measure on a single point $y$. We introduce continuous disintegrations as those which vary continuously in $y$. We present a necessary and sufficient condition for continuous disintegrations to exist for Gaussian measures on separable Banach spaces. For the example of continuous functions on a compact set, this condition takes a simple form and is satisfied for a wide class of applications, including stationary Gaussian processes. We also analyze how continuous disintegrations transform under absolutely-continuous changes of measure.
\end{abstract}

\section{IntRoduction}

Let $X$ and $Y$ be complete, separable metric spaces, with Borel $\sigma$-algebras $\mathcal{B}(X)$ and $\mathcal{B}(Y)$, and let $\mathbb{P}$ be a Radon probability measure on $X$. Let $\eta: X \rightarrow Y$ be a measurable function, and denote the push-forward measure of $\mathbb{P}$ on $Y$ by $\mathbb{P}_{Y}=\mathbb{P} \circ \eta^{-1}$. A disintegration (or regular conditional probability) of $\mathbb{P}$ with respect to $\eta$ is a map $Y \times \mathcal{B}(X) \rightarrow \mathbb{R}$ (denoted by $\left.(y, B) \mapsto \mathbb{P}^{y}(B)\right)$ such that:

- For all $y \in Y, \mathbb{P}^{y}$ is a probability measure on $\mathcal{B}(X)$.

- For all $B \in \mathcal{B}(X), y \mapsto \mathbb{P}^{y}(B)$ is a measurable function of $y \in Y$.

- The measure $\mathbb{P}^{y}$ is supported on the fiber of $y$. i.e., for $\mathbb{P}_{Y}$-almost every $y \in Y$, $\mathbb{P}^{y}\left(\eta^{-1}(y)\right)=1$, and

- For all integrable functions $f: X \rightarrow \mathbb{R}$,

$$
\int_{X} f(x) \mathrm{d} \mathbb{P}(x)=\int_{Y} \int_{\eta^{-1}(y)} f(x) \mathrm{d} \mathbb{P}^{y}(x) \mathrm{d} \mathbb{P}_{Y}(y) .
$$

Date: Spring 2010. 
Furthermore, if $\eta$ is continuous and $Y_{0}$ is a closed subset of $Y$ of full $\mathbb{P}_{Y}$-measure, we say that $\mathbb{P}^{y}$ is a continuous disintegration on $Y_{0}$ provided

- If $y_{n} \in Y_{0}$ and $y_{n} \rightarrow y$, then $\mathbb{P}^{y_{n}}$ converges weakly to $\mathbb{P}^{y}$.

While disintegrations exist in wide generality [1, 3], we are unaware of any discussion of continuous disintegrations. In the case where $X$ and $Y$ are finite-dimensional vector spaces, $\mathbb{P}$ is a Gaussian measure on $X$, and $\eta: X \rightarrow Y$ is a linear map, it is a simple exercise to show that $\mathbb{P}$ has a continuous disintegration $\mathbb{P}^{y}$ on $Y$ which is itself a Gaussian measure on $X$. For a Gaussian measure $\mathbb{P}$ on a separable Banach space $X$ and a continuous linear map $\eta: X \rightarrow Y$, Tarieladze and Vakhania [6] show that $\mathbb{P}$ admits a disintegration $\mathbb{P}^{y}$ which is a Gaussian measure for all $y$. Furthermore, when the push-forward measure $\mathbb{P}_{Y}$ has finite-dimensional support in $Y$, it quickly follows from their Theorem 3.11a that $\mathbb{P}^{y}$ is a continuous disintegration on the support.

In Section 2, we present some results for general measures on separable Banach spaces. Let $\mathbb{P}_{Y}$ be the push-forward measure of $\mathbb{P}$ under $\eta$, and let $K$ be the covariance operator of $\mathbb{P}$. The linear space $\eta K \eta^{*} Y^{*}$ is of critical importance to our study, as its closure $Y_{0}$ has full $\mathbb{P}_{Y}$-measure in $Y$. We define $M$ as the operator norm of $\eta^{-1}$ on $\eta K \eta^{*} Y^{*}$. In Section 3, we prove the main results of the paper: the finiteness of $M$ is a necessary and sufficient condition for a Gaussian measure $\mathbb{P}$ to admit a continuous disintegration $\mathbb{P}^{y}$ on $Y_{0}$.

In Section 4.1, we consider the question of continuous disintegrations for Gaussian stochastic processes (or random fields). When $X=C(U)$ is the space of real-valued continuous functions on a compact set $U \subseteq \mathbb{R}^{n}, M$ has a simple form involving the covariance function $c$ of the measure $\mathbb{P}$. In fact, $M$ is finite for many applications, including the case when $c$ is a stationary covariance function.

In Section 4.2, we analyze how continuous disintegrations transform under absolutelycontinuous changes of measure. If $\mu$ is a measure absolutely continuous to $\mathbb{P}$, in 
Section 4.2 we explicitly construct a disintegration $\mu^{y}$ using the measures $\mathbb{P}_{Y}$ and $\mathbb{P}^{y}$. Furthermore, if $\mathbb{P}^{y}$ is a continuous disintegration on $Y_{0}$ and the Radon-Nikodym derivative $\mathrm{d} \mu / \mathrm{d} \mathbb{P}$ is bounded and continuous on $X$, we show that there exists an open subset $U$ of $Y_{0}$ such that for all compact $K \subseteq U$, if $y_{n} \rightarrow y$ in $K$ then $\mu^{y_{n}} \rightarrow \mu^{y}$. We also provide an example illustrating that the continuous disintegration need not extend to the boundary of $U$.

We state and prove our results for Gaussian measures $\mathbb{P}$ with zero mean, but analogous statements hold when the mean is non-zero.

\section{General Remarks on Measures in Banach Spaces}

Let $X$ be a separable Banach space, and denote the Borel $\sigma$-algebra of $X$ by $\mathcal{B}(X)$. Continuous linear functionals of $X$ are measurable functions, hence random variables. Let $\mathbb{P}$ be a probability measure on $(X, \mathcal{B}(X))$ such that the continuous linear functionals have finite variance: $X^{*} \subseteq L^{2}(X, \mathcal{B}(X), \mathbb{P})$. These are the weakorder-two probability measures of [8, Section III.2], and it follows that there exist an element $m \in X$ and a continuous operator $K: X^{*} \rightarrow X$ for $\mathbb{P}$ such that

$$
\mathbb{E}(f)=f(m) \quad \text { and } \quad \mathbb{E}(f g)-f(m) g(m)=f(K g)
$$

for all $f, g \in X^{*}$. We call $m$ the mean and $K$ the covariance operator of $\mathbb{P}$.

We define the support of $\mathbb{P}$ to be the smallest closed set in $X$ of full measure, and we denote this by supp $\mathbb{P}$. In [7], Vakhania proves that the support is described by the mean and covariance operator of $\mathbb{P}$ :

\section{Proposition 1.}

$$
\operatorname{supp} \mathbb{P} \subseteq m+\overline{K X^{*}}
$$

Consequently, $\mathbb{P}\left(m+\overline{K X^{*}}\right)=1$. If $\mathbb{P}$ is a Gaussian measure, then supp $\mathbb{P}=m+\overline{K X^{*}}$.

For the remainder of this section, we assume that the mean of $\mathbb{P}$ is zero. 
Let $Y$ be a separable Banach space, and let $\eta: X \rightarrow Y$ be a continuous linear map. Denote by $\mathcal{B}(Y)$ the Borel $\sigma$-algebra of $Y$. Let $\mathbb{P}_{Y}$ be the push-forward measure on $Y$ of $\mathbb{P}$ :

$$
\mathbb{P}_{Y}(B)=\mathbb{P}\left(\eta^{-1}(B)\right)
$$

for any Borel set $B \in \mathcal{B}(Y)$. The measure $\mathbb{P}_{Y}$ satisfies the change of variable formula

$$
\int_{\eta^{-1}(B)} g(\eta x) \mathrm{d} \mathbb{P}(x)=\int_{B} g(y) d \mathbb{P}_{Y}(y),
$$

for any integrable $g: Y \rightarrow \mathbb{R}$. Consequently, $\mathbb{P}_{Y}$ has mean zero and covariance operator $\eta K \eta^{*}$.

The covariance operator $K$ defines a symmetric inner product $\langle f, g\rangle=f(K g)$ on $X^{*}$. For a subspace $B$ of $X^{*}$, let $B^{\perp}$ be the space of functionals uncorrelated with $B$ :

$$
B^{\perp}=\left\{f \in X^{*}: f(K g)=0 \text { for all } g \in B\right\}
$$

Lemma 2. When restricted to the subspace $K X^{*}$ of $X$, the map $\eta$ has kernel $K\left(\eta^{*} Y^{*}\right)^{\perp}$. Consequently, on $K \eta^{*} Y^{*}, \eta$ is injective.

Proof. Let $f \in X^{*}$, and suppose that $\eta(K f)=0$ in $Y$. Then for all $e \in Y^{*}$,

$$
0=e(\eta K f)=\left\langle\eta^{*} e, f\right\rangle
$$

thus $f \in\left(\eta^{*} Y^{*}\right)^{\perp}$.

Define the linear map $m: \eta K \eta^{*} Y^{*} \rightarrow X$ by

$$
m(y)=\eta^{-1}(y)
$$

This is well-defined by Lemma 2, and has (possibly infinite) operator norm

$$
M:=\|m\|_{\mathrm{op}}=\sup _{e \in Y^{*}}\left\{\frac{\left\|K \eta^{*} e\right\|_{X}}{\left\|\eta K \eta^{*} e\right\|_{Y}}: K \eta^{*} e \neq 0\right\}
$$


In fact, since $\eta K \eta^{*}$ generates an inner product on $Y^{*}$, it satisfies the Schwarz inequality $[2]$

$$
\left|e^{\prime} \eta K \eta^{*} e\right|^{2} \leq\left|e^{\prime} \eta K \eta^{*} e^{\prime}\right|\left|e \eta K \eta^{*} e\right|
$$

Thus $e^{\prime}\left(\eta K \eta^{*} e\right) \neq 0$ for some $e^{\prime} \in Y^{*}$ exactly if $e\left(\eta K \eta^{*} e\right) \neq 0$, and we have the simpler expression

$$
M=\sup _{e \in Y^{*}}\left\{\frac{\left\|K \eta^{*} e\right\|_{X}}{\left\|\eta K \eta^{*} e\right\|_{Y}}: e\left(\eta K \eta^{*} e\right) \neq 0\right\} .
$$

Let

$$
Y_{0}=\overline{\eta K \eta^{*} Y^{*}}
$$

Since $\eta K \eta^{*}$ is the covariance operator of $\mathbb{P}_{Y}$, Proposition 1 implies that $\operatorname{supp} \mathbb{P}_{Y} \subseteq Y_{0}$ so $\mathbb{P}_{Y}\left(Y_{0}\right)=1$.

We suppose for the remainder of this section that $M<\infty$. This makes $m$ continuous on $\eta K \eta^{*} Y^{*}$, and we extend $m$ continuously to all of $Y_{0}$. Note that $m$ is a continuous function defined $\mathbb{P}_{Y^{-}}$-almost everywhere, and satisfies $\eta(m(y))=y$ for all $y \in Y_{0}$.

Lemma 3. The operator $\hat{K}: X^{*} \rightarrow X$ given by the formula

$$
\hat{K}=K-K \eta^{*} m^{*}
$$

is well-defined. Furthermore,

$$
m \eta K \eta^{*} m^{*}=K \eta^{*} m^{*}
$$

We call $m(y)$ the conditional mean and $\hat{K}$ the conditional covariance operator of $\mathbb{P}$ with respect to $\eta$. This nomenclature will be clear in the context of Gaussian measures in the next section. 
Proof. Let $H$ be the Hilbert space completion of the space $X^{*}$ under the inner product generated by $K$, and let $\iota^{*}: X^{*} \hookrightarrow H$ be the inclusion map. Define the unitary map $\iota: H \rightarrow X$ first on the dense subspace $\iota^{*} X^{*}$ by $\iota\left(\iota^{*} f\right)=K f$, then extend it continuously to all of $H$. Thus $K$ factors as $\iota \iota^{*}$.

Let $H_{Y}$ be the completion of $\iota^{*} \eta^{*} Y^{*}$ in $H$, and let $\pi: H \rightarrow H$ be the orthogonal projection onto the subspace $H_{Y}$. We claim that the two continuous maps $m \eta \iota$ and $\iota \pi$ from $H$ to $X$ are equal. It suffices to check the action on the dense subspaces $\iota^{*} \eta^{*} Y^{*} \subseteq H_{Y}$ and $\iota^{*}\left(\eta^{*} Y^{*}\right)^{\perp} \subseteq H_{Y}^{\perp}:$

$$
(m \eta \iota-\iota \pi) \iota^{*} \eta^{*} Y^{*}=m \eta K \eta^{*} Y^{*}-K \eta^{*} Y^{*}=0
$$

since $\pi$ is the identity on $\iota^{*} \eta^{*} Y^{*}$ and $m \circ \eta$ is the identity on $K \eta^{*} Y^{*}$, and

$$
(m \eta \iota-\iota \pi) \iota^{*}\left(\eta^{*} Y^{*}\right)^{\perp}=m \eta K\left(\eta^{*} Y^{*}\right)^{\perp}-0=0
$$

since $\pi$ kills $\iota^{*}\left(\eta^{*} Y^{*}\right)^{\perp}$ and $K\left(\eta^{*} Y^{*}\right)^{\perp} \subseteq \operatorname{ker} \eta$ by Lemma 2. Thus $m \eta \iota=\iota \pi$ on $H$.

By duality, the adjoint maps $\iota^{*} \eta^{*} m^{*}$ and $\pi \iota^{*}$ from $X^{*}$ to $H$ are also equal, so

$$
\hat{K}=K-K \eta^{*} m^{*}=K-\iota \iota^{*} \eta^{*} m^{*}=K-\iota \pi \iota^{*}
$$

is well-defined.

For the proof of equation (4), observe that

$$
m \eta K \eta^{*} m^{*}=m \eta \iota \iota^{*} \eta^{*} m^{*}=\iota \pi^{2} \iota^{*}=\iota \pi \iota^{*}=K \eta^{*} m^{*} .
$$

Lemma 4. The space $\hat{K} X^{*}$ is in the kernel of $\eta$. Consequently,

$$
\eta\left(m(y)+\overline{\hat{K} X^{*}}\right)=y
$$


for all $y \in Y_{0}$.

Proof. Let $f \in X^{*}$. The claim is proved if we verify that $e(\eta \hat{K} f)=0$ for all $e \in Y^{*}$. Recall that $\eta \circ m$ is the identity on $Y_{0}$ and $m \circ \eta$ is the identity on $K \eta^{*} Y^{*}$. Thus

$$
e(\eta \hat{K} f)=e(\eta K f)-e\left(\eta K \eta^{*} m^{*} f\right)=f\left(K \eta^{*} e\right)-f\left(m \eta K \eta^{*} e\right)=0
$$

by the symmetry of $K$.

\section{Gaussian Disintegrations}

A measure $\mathbb{P}$ on a Banach space $X$ is said to be Gaussian if each continuous linear functional $f \in X^{*}$ is a real-valued Gaussian random variable. A Gaussian measure $\mathbb{P}$ is described completely by its mean $m$ and covariance operator $K$; by Proposition 1 , its support is the entire linear space $m+\overline{K X^{*}}$.

Supposing that $M<\infty$, we exploit the Gaussian structure in three ways to construct a continuous disintegration $\mathbb{P}^{y}$ on $Y_{0}$. First, once we have the conditional mean $m(y)$ and conditional covariance $\hat{K}$ as in the preceding section, we define $\mathbb{P}^{y}$ as the Gaussian measure with mean $m(y)$ and covariance $\hat{K}$. This is the infinite-dimensional analogue of the fact that conditioned finite-dimensional Gaussians are still Gaussian.

Second, instead of verifying the disintegration equation (1) directly, we verify an equivalent identity involving characteristic functionals. This is a simple, algebraic computation in the Gaussian context, since the characteristic functionals of Gaussian measures have an explicit form. Third, to show that $\mathbb{P}^{y}$ varies continuously in $y$, we take advantage of the fact that all Gaussian measures with the same covariance operator are simply translations of the zero-mean Gaussian. This makes weak convergence easy to prove, which we do in the following lemma. 
Lemma 5. Let $X$ be a Banach space, and let $m_{n} \rightarrow m$ in $X$. Let $\mathbb{P}_{n}$ and $\mathbb{P}$ be Gaussian measures with means $m_{n}$ and $m$, respectively, and the same covariance operator $K$. Then $\mathbb{P}_{n} \rightarrow \mathbb{P}$ weakly.

Proof. Let $\mathbb{P}_{0}$ be the Gaussian measure with mean zero and covariance $K$. If $f$ is a continuous, bounded function on $X$, then

$\lim _{n \rightarrow \infty} \int_{X} f(x) d \mathbb{P}_{n}(x)=\lim _{n \rightarrow \infty} \int_{X} f\left(x+m_{n}\right) d \mathbb{P}_{0}(x)=\int_{X} f(x+m) d \mathbb{P}_{0}(x)=\int_{X} f(x) \mathrm{d} \mathbb{P}(x)$

by the Bounded Convergence Theorem.

Theorem 6. Let $X$ and $Y$ be separable Banach spaces, and $\eta: X \rightarrow Y$ a continuous linear map. Let $\mathbb{P}$ be a Gaussian measure on $X$ with mean zero and covariance operator $K$, and let $\mathbb{P}_{Y}$ be the push-forward measure of $\mathbb{P}$ on $Y$. Suppose that

$$
M=\sup _{e \in Y^{*}}\left\{\frac{\left\|K \eta^{*} e\right\|_{X}}{\left\|\eta K \eta^{*} e\right\|_{Y}}: e\left(\eta K \eta^{*} e\right) \neq 0\right\}<\infty .
$$

There exists a continuous disintegration $\mathbb{P}^{y}$ on $Y_{0}=\overline{\eta K \eta^{*} Y^{*}}$. Furthermore, there exists a continuous linear operator $m: Y_{0} \rightarrow X$ such that for all $y \in Y_{0}, \mathbb{P}^{y}$ is the Gaussian measure with mean $m(y)$ and covariance operator $\hat{K}=K-K \eta^{*} m^{*}$.

Proof. Define the conditional mean $m: Y_{0} \rightarrow X$ as in the preceding section. We assume $M<\infty$ so the operator $m$ is continuous on $Y_{0}$. Let $\hat{K}=K-K \eta^{*} m^{*}$ be the conditional covariance operator. By assumption, $K$ is a covariance operator for a Gaussian measure; since $\hat{K} \leq K, \hat{K}$ is also a Gaussian covariance operator [6, Proposition 3.9]. Let $\mathbb{P}^{y}$ be the Gaussian measure on $X$ with mean $m(y)$ and covariance operator $\hat{K}$. To show that $\mathbb{P}^{y}$ is a disintegration with respect to $\eta$, we must verify that $\mathbb{P}^{y}\left(\eta^{-1}(y)\right)=1$ and the disintegration equation (1).

By Proposition 1, the support of $\mathbb{P}^{y}$ is

$$
m(y)+\overline{\hat{K} X^{*}} .
$$


By Lemma $4, \eta\left(m(y)+\overline{\hat{K} X^{*}}\right)=y$, hence $\mathbb{P}^{y}\left(\eta^{-1}(y)\right)=1$.

The characteristic functional of a measure $\mu$ on $X$ is the map $\hat{\mu}: X^{*} \rightarrow \mathbb{R}$ defined by

$$
\hat{\mu}(f)=\int_{X} \mathrm{e}^{\mathrm{i} f(x)} \mathrm{d} \mu(x)
$$

for all $f \in X^{*}$. If $\mu$ is Gaussian with mean $m$ and covariance operator $K$, then its characteristic functional has the form [6, Lemma 3.6]

$$
\hat{\mu}(f)=\mathrm{e}^{\mathrm{i} f(m)-f(K f) / 2}
$$

There is an equivalent formulation of the disintegration equation (1) using characteristic functionals [6, Proposition 3.2]: $\mathbb{P}^{y}$ satisfies (1) if and only if

$$
\hat{\mathbb{P}}(f)=\int_{Y} \hat{\mathbb{P}}^{y}(f) d \mathbb{P}_{Y}(y)
$$

for all $f \in X^{*}$. Since $\mathbb{P}$ has mean zero and covariance operator $K, \hat{\mathbb{P}}(f)=\mathrm{e}^{-f(K f) / 2}$. Thus we compute

$$
\begin{aligned}
\int_{Y} \hat{\mathbb{P}}^{y}(f) d \mathbb{P}_{Y}(y) & =\int_{Y} \mathrm{e}^{\mathrm{i} f(m(y))-f(\hat{K} f) / 2} \mathrm{~d} \mathbb{P}_{Y}(y) \\
& =\mathrm{e}^{-f(K f) / 2+f\left(K \eta^{*} m^{*} f\right) / 2} \int_{Y} \mathrm{e}^{\mathrm{i} f(m(y))} \mathrm{d} \mathbb{P}_{Y}(y) \\
& =\mathrm{e}^{-f(K f) / 2+f\left(K \eta^{*} m^{*} f\right) / 2} \int_{X} \mathrm{e}^{\mathrm{i} f(m(\eta(x)))} \mathrm{d} \mathbb{P}(x) .
\end{aligned}
$$

by the change of variable formula (2). The latter integral is itself the action of the characteristic functional $\hat{\mathbb{P}}$ on $\eta^{*} m^{*} f$ :

$\hat{\mathbb{P}}\left(\eta^{*} m^{*} f\right)=\exp \left(-\eta^{*} m^{*} f\left(K \eta^{*} m^{*} f\right) / 2\right)=\exp \left(-f\left(m \eta K \eta^{*} m^{*} f\right) / 2\right)=\exp \left(-f\left(K \eta^{*} m^{*} f\right) / 2\right)$

by equation (4). This cancels with the $\exp \left(f\left(K \eta^{*} m^{*} f\right) / 2\right)$ term in (5), proving that $\mathbb{P}^{y}$ is a disintegration. 
Finally, we verify that $\mathbb{P}^{y}$ is a continuous disintegration on $Y_{0}$. If $y_{n} \rightarrow y$, then $m\left(y_{n}\right) \rightarrow m(y)$ since $m$ is continuous on $Y_{0}$. The measures $\mathbb{P}^{y}$ all have the same covariance operator $\hat{K}$, so Lemma 5 applies and $\mathbb{P}^{y_{n}} \rightarrow \mathbb{P}^{y}$ weakly. This completes the proof.

Having shown that $M<\infty$ is a sufficient condition for there to exist a continuous disintegration of a Gaussian measure, we now show it is a necessary condition.

Theorem 7. Let $X$ and $Y$ be separable Banach spaces, and $\eta: X \rightarrow Y$ a continuous linear map. Let $\mathbb{P}$ be a Gaussian measure on $X$ with mean zero and covariance operator $K$, and let $\mathbb{P}_{Y}$ be the push-forward measure of $\mathbb{P}$ on $Y$. Suppose that

$$
M=\sup _{e \in Y^{*}}\left\{\frac{\left\|K \eta^{*} e\right\|_{X}}{\left\|\eta K \eta^{*} e\right\|_{Y}}: e\left(\eta K \eta^{*} e\right) \neq 0\right\}=\infty .
$$

For any closed set $Y_{0}$ of full $\mathbb{P}_{Y}$-measure, there does not exist a continuous disintegration $\mathbb{P}^{y}$ on $Y_{0}$.

Proof. Let $Y_{0}$ be a closed subset of $Y$ of full $\mathbb{P}_{Y}$-measure, and suppose $\tilde{\mathbb{P}}^{y}$ is a continuous disintegration of $\mathbb{P}$ on $Y_{0}$.

The main result of [6, Theorem 3.11] is that there exist a Borel-measurable map $m: Y \rightarrow X$ and a Gaussian covariance operator $\hat{K}$ such that the Gaussian measure $\mathbb{P}^{y}$ with mean $m(y)$ and covariance $\hat{K}$ is a disintegration of $\mathbb{P}$. Furthermore, there exists a vector subspace $Y_{1}$ of $Y$ of full $\mathbb{P}_{Y}$-measure such that the restriction of $m$ to $Y_{1}$ is Borel measurable and $\eta(m(y))=y$ for all $y \in Y_{1}$.

Disintegrations are unique up to sets of measure zero [6, Theorem 2.4], so there exists a set $Y_{2}$ of $Y$ of full $\mathbb{P}_{Y^{-}}$-measure such that $\tilde{\mathbb{P}}^{y}=\mathbb{P}^{y}$ for all $y \in Y_{2}$. Let

$$
Y^{\prime}=\overline{Y_{0} \cap Y_{1} \cap Y_{2}}
$$


so that $\mathbb{P}^{y}$ is a continuous disintegration of $\mathbb{P}$ on $Y^{\prime}$. Since $Y^{\prime}$ is a closed set of full $\mathbb{P}_{Y}$-measure, it contains the linear space supp $\mathbb{P}_{Y}=\overline{\eta K \eta^{*} Y^{*}}$ as a subset.

Lemma 8. There exists a sequence $y_{n} \in \eta K \eta^{*} Y^{*}$ such that $y_{n} \rightarrow 0$ but $\left\|m\left(y_{n}\right)\right\|_{X} \geq 1$ for all $n$. Consequently, $m$ is discontinuous on $Y^{\prime}$.

Furthermore, the distance in $X$ from $m\left(y_{n}\right)$ to $\overline{\hat{K} X^{*}}$ is at least 1 for all $n$.

Proof. As in the proof of Lemma 3, let $H$ be the Hilbert space completion of the space $X^{*}$ under the inner product generated by $K$, and let $\iota^{*}: X^{*} \hookrightarrow H$ be the inclusion map. Define the unitary map $\iota: H \rightarrow X$ first on the dense subspace $\iota^{*} X^{*}$ by $\iota\left(\iota^{*} f\right)=K f$, then extend it continuously to all of $H$.

Let $H_{Y}$ be the completion of $\iota^{*} \eta^{*} Y^{*}$ in $H$. Choose $e_{i} \in Y^{*}$ so that $h_{i}=\iota^{*} \eta^{*} e_{i}$ is an orthonormal basis in $H_{Y}$. For all $y \in Y^{\prime}$,

$$
m(y)=\sum_{i=1}^{\infty} e_{i}(y) K \eta^{*} e_{i}=\iota\left(\sum_{i=1}^{\infty} e_{i}(y) h_{i}\right)
$$

this follows from the proof of [6, Theorem 3.11, Case 3]. If $\pi: H \rightarrow H$ is the orthogonal projection onto $H_{Y}$ in $H$, this formula implies that $m \eta \iota=\iota \pi$ on $H$. Thus for $g \in Y^{*}$,

$$
\left\|m\left(\eta K \eta^{*} g\right)\right\|_{X}=\left\|(m \eta \iota) \iota^{*} \eta^{*} g\right\|_{X}=\left\|\iota \pi \iota^{*} \eta^{*} g\right\|_{X}=\left\|K \eta^{*} g\right\|_{X}
$$

since $\pi$ is the identity on $\iota^{*} \eta^{*} Y^{*}$.

Since $M=\infty$, there exist $g_{n} \in Y^{*}$ such that

$$
\left\|K \eta^{*} g_{n}\right\|_{X} \geq n\left\|\eta K \eta^{*} g_{n}\right\|_{Y}
$$

Setting

$$
y_{n}=\frac{\eta K \eta^{*} g_{n}}{n\left\|\eta K \eta^{*} g_{n}\right\|}
$$

and applying (6) completes the proof that $\left\|m\left(y_{n}\right)\right\| \geq 1$. 
It also follows from the proof of [6, Theorem 3.11, Case 3] that

$$
\hat{K}=K-\iota \pi \iota^{*}
$$

If $g \in Y^{*}$ and $f \in X^{*}$, then for $y=\eta K \eta^{*} g$,

$$
\begin{aligned}
\|m(y)-\hat{K} f\|_{X}^{2} & =\left\|\iota \iota^{*} \eta^{*} g-\left(\iota \iota^{*} f-\iota \pi \iota^{*} f\right)\right\|_{X}^{2} \\
& =\left\|\iota^{*} \eta^{*} g-(\mathrm{id}-\pi) \iota^{*} f\right\|_{H}^{2} \\
& =\left\|\iota^{*} \eta^{*} g\right\|_{H}^{2}+\left\|(\mathrm{id}-\pi) \iota^{*} f\right\|_{H}^{2} \\
& \geq\|m(y)\|_{X}^{2}+0,
\end{aligned}
$$

by the Pythagorean Theorem [2], since id $-\pi$ is the orthogonal projection onto $H_{Y}^{\perp}$. Plugging in $y_{n}$ as above completes the proof of the second claim.

Since $y_{n} \rightarrow 0$ in $\eta K \eta^{*} Y^{*} \subseteq Y^{\prime}$ and $\mathbb{P}^{y}$ is a continuous disintegration on $Y^{\prime}, \mathbb{P}^{y_{n}} \rightarrow \mathbb{P}^{0}$ weakly. By Proposition $1, \mathbb{P}^{0}$ is supported on $\overline{\hat{K} X^{*}}$. Thus the open $\frac{1}{2}$-neighborhood of $\overline{\hat{K} X^{*}}$,

$$
U=\left\{x \in X:\left\|x-x^{\prime}\right\|<\frac{1}{2} \text { for some } x^{\prime} \in \overline{\hat{K} X^{*}}\right\}
$$

has full $\mathbb{P}^{0}$-measure. Since $\mathbb{P}^{y_{n}} \rightarrow \mathbb{P}^{0}$ weakly and $U$ is open, $\lim \inf \mathbb{P}^{y_{n}}(U) \geq \mathbb{P}^{0}(U)=$ 1. However, $\mathbb{P}^{y_{n}}$ is supported on $m\left(y_{n}\right)+\overline{\hat{K} X^{*}}$, which is distance at least 1 from $\overline{\hat{K} X^{*}}$ by the preceding lemma, a contradiction.

\section{Applications}

4.1. Gaussian Processes. Let $U \subseteq \mathbb{R}^{n}$ be compact, and consider $X=C(U)$ the space of real-valued continuous functions on $U$ with the supremum norm

$$
\|x\|_{X}=\sup _{t \in U}|x(t)| .
$$


A stochastic process is just an $X$-valued random variable (for example, a standard Wiener process is a $C([0,1])$-valued random variable). In this context, $M$ is easily computable, and in fact is finite for many applications.

For $t \in U$, let $\delta_{t} \in X^{*}$ be the evaluation functional, defined by $\delta_{t} x=x(t)$. The Riesz representation theorem [2] says that $X^{*}$ is the space of Radon measures on $U$, hence $\delta_{t}$ is the unit point mass measure at $t$.

We recall that a function $c: U \times U \rightarrow \mathbb{R}$ is positive-definite if for any $t_{1}, \ldots, t_{n} \in U$, the $n \times n$ matrix given by $c\left(t_{i}, t_{j}\right)$ is positive-definite. We say that a function $c$ is stationary if $c(s, t)=c(s-v, t-v)$ for any $v \in \mathbb{R}^{n}$ such that $(s-v, t-v) \in U \times U$.

Proposition 9. Suppose $U \subseteq \mathbb{R}^{n}$ is compact, and let $V$ be a closed subset of $U$. Let $X=C(U)$ and $Y=C(V)$. Let $\eta: X \rightarrow Y$ be the restriction map, defined by $(\eta x)(t)=x(t)$ for $t \in V$. Let $c: U \times U \rightarrow \mathbb{R}$ be a continuous, positive-definite function, and define the integral operator $K: X^{*} \rightarrow X$ by

$$
(K \mu)(t)=\int_{U} c(t, s) \mathrm{d} \mu(s)
$$

for all Radon measures $\mu \in X^{*}$ and $t \in U$. Suppose $\mathbb{P}$ is a measure with mean zero and covariance operator $K$. Then

$$
M=\sup _{s \in V}\left\{\frac{\sup _{t \in U}|c(s, t)|}{\sup _{s^{\prime} \in V}\left|c\left(s, s^{\prime}\right)\right|}: c(s, s) \neq 0\right\} .
$$

Equivalently, $M$ is the minimum $M^{\prime} \geq 1$ for which

$$
\sup _{t \in U} c(s, t) \leq M^{\prime} \sup _{s^{\prime} \in V} c\left(s, s^{\prime}\right)
$$

for all $s \in V$.

If $c(s, t)$ attains its maximum at $t=s$ for all $t, s$, then $M=1$.

If $c$ is stationary, then $M=1$.

If $c$ is bounded from below on $V \times V$, then $M<\infty$. 
Proof. The linear span of $\left\{\delta_{s}\right\}_{s \in V}$ is dense in $Y^{*}[5]$, so when we calculate $M$ as in (3), it suffices to consider only functionals of the form $e=\delta_{s}$. Furthermore, $K \eta^{*} \delta_{s}$ is the function $c(s, \cdot)$. This proves $(7)$.

If $c(s, s) \geq c(s, t)$ for all $s \in V$ and $t \in U$, then the suprema in (7) are attained with both $t$ and $s^{\prime}$ equal to $s$, hence $M=1$. If $c$ is stationary, then by the Schwarz inequality,

$$
c(s, t)^{2} \leq c(s, s) c(t, t)=c(s, s) c(t-(t-s), t-(t-s))=c(s, s)^{2}
$$

for all $s$ and $t$, so $c(s, t)$ attains its maximum when $s$ and $t$ are equal, hence $M=1$. The final assertion is trivial, since as a continuous function, $c$ is bounded above on $V \times U$

Write $c_{s}(t)=c(s, t)$ and

$$
Y_{0}=\overline{\operatorname{span}\left\{c_{s}\right\}} \subseteq Y
$$

where the span is over $s \in V$. Suppose that $M<\infty$, and define the continuous operator $m: Y_{0} \rightarrow X$ by

$$
m\left(c_{s}\right)(t)=c_{s}(t)
$$

extending linearly and continuously to all of $Y_{0}$. For $t \in U$, denote by $\left.c_{t}\right|_{V}$ the restriction of $c_{t}$ to $V$, and let $a_{t} \in X$ be the function $a_{t}=m\left(\left.c_{t}\right|_{V}\right)$. Define the positive-definite function $\hat{c}: U \times U \rightarrow \mathbb{R}$ by

$$
\hat{c}\left(t, t^{\prime}\right)=c\left(t, t^{\prime}\right)-a_{t}\left(t^{\prime}\right)
$$

Corollary 10. Let $X=C(U), Y=C(V)$, and $\eta: X \rightarrow Y$ be the restriction map. Suppose $\mathbb{P}$ is a Gaussian measure on $X$ with mean zero and covariance function $c(s, t)$. Under the push-forward measure $\mathbb{P}_{Y}$ of $\mathbb{P}$, the space $Y_{0}$ has full measure in $Y$. 
Define $M$ as in (7). The Gaussian measure $\mathbb{P}$ has a continuous disintegration $\mathbb{P}^{y}$ on $Y_{0}$ if and only if $M<\infty$. Furthermore, $\mathbb{P}^{y}$ is the Gaussian measure on $X$ with mean $m(y)$ and covariance function $\hat{c}$.

4.2. Absolute Continuity of Measures. In this section, we make no assumption that $X$ and $Y$ are Banach spaces, nor that $\mathbb{P}$ is a Gaussian measure.

Theorem 11. Let $X$ and $Y$ be complete, separable metric spaces, with $\eta: X \rightarrow Y$ a continuous linear map. Let $\mathbb{P}$ be a Radon measure on $X$ with a disintegration $\mathbb{P}^{y}$. Suppose $\mu \ll \mathbb{P}$, and denote the push-forward measures of $\mu$ and $\mathbb{P}$ by $\mu_{Y}$ and $\mathbb{P}_{Y}$, respectively. Then $\mu_{Y} \ll \mathbb{P}_{Y}$; there exists a disintegration $\mu^{y}$ of $\mu$; and $\mu^{y} \ll \mathbb{P}^{y}$ for $\mathbb{P}_{Y}$-almost every $y$.

Furthermore, let $\mathbb{P}^{y}$ be a continuous disintegration on $Y_{0} \subseteq Y$, and suppose that $\rho(x)=\frac{\mathrm{d} \mu}{\mathrm{d} \mathbb{P}}(x)$ is bounded and continuous on $\operatorname{supp} \mathbb{P} \subseteq X$. Then $\rho_{Y}(y):=\frac{\mathrm{d} \mu_{Y}}{\mathrm{dP}_{Y}}(y)$ is continuous on $Y_{0} ; \mu^{y} \ll \mathbb{P}^{y}$ on the open subset $U=\left\{y: \rho_{Y}(y)>0\right\} \subseteq Y_{0}$ of full $\mu_{Y}$-measure; and for all compact $K \subseteq U$, if $y_{n} \rightarrow y$ in $K$ then $\mu^{y_{n}} \rightarrow \mu^{y}$ weakly.

Proof. Write $\rho(x)=\frac{\mathrm{d} \mu}{\mathrm{d} \mathbb{P}}(x)$. Let $\mathbb{P}_{Y}$ and $\mu_{Y}$ be the push-forward measures of $\mathbb{P}$ and $\mu$, respectively. Using the disintegration equation for $\mathbb{P}^{y}$ yields

$$
\mu_{Y}(B)=\mu\left(\eta^{-1}(B)\right)=\int_{\eta^{-1}(B)} \rho(x) \mathrm{d} \mathbb{P}(x)=\int_{B} \int_{\eta^{-1}(y)} \rho(x) \mathrm{d} \mathbb{P}^{y}(x) \mathrm{d} \mathbb{P}_{Y}(y)
$$

for every $B \in \mathcal{B}(Y)$, hence $\mu_{Y} \ll \mathbb{P}_{Y}$ with

$$
\rho_{Y}(y):=\frac{\mathrm{d} \mu_{Y}}{\mathrm{dP}_{Y}}(y)=\int_{\eta^{-1}(y)} \rho(x) \mathrm{d} \mathbb{P}^{y}(x)
$$

for $\mathbb{P}_{Y}$-almost every $y$. Since $\rho_{Y}(y)=0$ if and only if $\rho(x)=0$ for $\mathbb{P}^{y}$-almost every $x$, the measure $\mu^{y} \ll \mathbb{P}^{y}$ with Radon-Nikodym derivative

$$
\rho^{y}(x):=\frac{\mathrm{d} \mu^{y}}{\mathrm{~d} \mathbb{P} y}(x)=\frac{\rho(x)}{\rho_{Y}(y)}
$$


is well-defined on the set $\left\{y: \rho_{Y}(y)>0\right\}$ of full $\mu_{Y}$-measure.

We verify that $\mu^{y}$ satisfies the disintegration equation (1) for $\mu$ :

$$
\begin{aligned}
\int_{Y} \int_{\eta^{-1}(y)} f(x) \mathrm{d} \mu^{y}(x) \mathrm{d} \mu_{Y}(y) & =\int_{Y} \int_{\eta^{-1}(y)} f(x) \frac{\rho(x)}{\rho_{Y}(y)} \mathrm{d} \mathbb{P}^{y}(x) \mathrm{d} \mu_{Y}(y) \\
& =\int_{Y} \int_{\eta^{-1}(y)} f(x) \rho(x) \mathrm{d} \mathbb{P}^{y}(x) \mathrm{dP}_{Y}(y) \\
& =\int_{X} f(x) \rho(x) \mathrm{d} \mathbb{P}(x) \\
& =\int_{X} f(x) \mathrm{d} \mu(x)
\end{aligned}
$$

for all measurable $f$.

Now, suppose that $\mathbb{P}^{y}$ is a continuous disintegration on $Y_{0}$ and that $\rho(x)$ is continuous and bounded. By (8), this implies that the function $\rho_{Y}(y)$ is defined for all $y \in Y_{0}$ and is furthermore continuous. Thus $U=\left\{y \in Y_{0}: \rho_{Y}(y)>0\right\}$ is open in the subspace topology of $Y_{0}$.

Let $K$ be a compact subset of $U$. Suppose $y_{n} \rightarrow y$ in $K$, and let $f$ be a continuous, bounded function on $\operatorname{supp} \mathbb{P}$. Then

$$
\begin{aligned}
\left|\int f \mathrm{~d} \mu^{y_{n}}-\int f \mathrm{~d} \mu^{y}\right| & =\left|\int f \rho^{y_{n}} \mathrm{~d} \mathbb{P}^{y_{n}}-\int f \rho^{y} \mathrm{~d} \mathbb{P}^{y}\right| \\
& \leq \int|f|\left|\rho^{y_{n}}-\rho^{y}\right| \mathrm{d} \mathbb{P}^{y_{n}}+\left|\int f \rho^{y} \mathrm{~d} \mathbb{P}^{y_{n}}-\int f \rho^{y} \mathrm{~d} \mathbb{P}^{y}\right| \\
& \leq\left|\frac{1}{\rho_{Y}\left(y_{n}\right)}-\frac{1}{\rho_{Y}(y)}\right| \sup |f \rho|+\left|\int f \rho^{y} \mathrm{~d} \mathbb{P}^{y_{n}}-\int f \rho^{y} \mathrm{~d} \mathbb{P}^{y}\right|
\end{aligned}
$$

The first term goes to zero since $1 / \rho_{Y}(y)$ is continuous on $K$, and the second term goes to zero since $f \rho^{y}$ is a bounded, continuous function on $\operatorname{supp} \mathbb{P}$ and $\mathbb{P}^{y_{n}} \rightarrow \mathbb{P}^{y}$ weakly.

There need not exist a continuous disintegration $\mu^{y}$ extending to the boundary of $U$. Consider the probability space $X=[0,1] \times[0,2]$ with uniform measure, and let 
$Y=[0,2]$ with $\eta: X \rightarrow Y$ the projection onto the second component. Let $\rho(x, y)$ be a continuous density function satisfying

$$
\rho(x, y)=0 \text { if and only if } 1-x \leq y \leq 2-x
$$

Here, $U=[0,1) \cup(1,2] \subseteq Y$. As $y \rightarrow 1$ from below, the measures $\mu^{y}$ concentrate on the point $(x, y)=(0,1)$; as $y \rightarrow 1$ from above, they concentrate on $(x, y)=(1,1)$. Thus there is not a unique measure $\mu^{1}$ on $X$ such that $\mu^{y} \rightarrow \mu^{1}$ as $y \rightarrow 1$.

While in the above example, the sequence $\mu^{y_{n}}$ is tight, this need not be true if the probability space $X$ is not compact. Let $X=\mathbb{R}^{2}$ and $Y=[0,1]$ with $\eta: X \rightarrow Y$ the projection map. Denote by $\mathcal{N}(m)$ the one-dimensional Gaussian with mean $m$ and variance 1 , and $\mathcal{U}$ the uniform measure on $[0,1]$, and let $\mathbb{P}=\mathcal{N}(0) \times \mathcal{U}$. Let $\rho(x, y)=\frac{1}{Z} \mathrm{e}^{-1 / y}$, where $Z$ is the normalization constant so that $\rho \mathrm{d} \mathbb{P}$ is a probability measure. The reader may verify that $\mu^{y}=\mathcal{N}\left(-\frac{1}{y}\right) \times \delta_{y}$ on $X$, which is not tight as $y \rightarrow 0$.

Acknowledgements. The author thanks Janek Wehr and Joe Watkins for many useful discussions and helpful feedback, as well as Mark Meckes [4] for the simple proof of Lemma 5 .

\section{REFERENCES}

[1] R. Durrett. Probability: theory and examples. Duxbury Press Belmont, CA, 1996.

[2] GB Folland. Real Analysis: Modern Techniques and Their Applications. Wiley-Interscience, 1999.

[3] D. Leão Jr, M. Fragoso, and P. Ruffino. Regular conditional probability, disintegration of probability and Radon spaces. Proyecciones (Antofagasta), 23(1), 2004.

[4] M. Meckes. Convergence of Gaussian Measures. MathOverflow: http://mathoverflow.net/ questions/16422, 2010.

[5] M. Reed and B. Simon. Methods of Modern Mathematical Physics. Academic Press, 1981. 
[6] V. Tarieladze and N. Vakhania. Disintegration of Gaussian measures and average-case optimal algorithms. Journal of Complexity, 23(4-6):851-866, 2007.

[7] NN Vakhania. The topological support of Gaussian measure in Banach space. Nagoya Math. J, $57: 59-63,1975$.

[8] NN Vakhania, VI Tarieladze, and SA Chobanyan. Probability distributions on Banach spaces. Transl. from the Russian by Wojbor A. Woyczynski. Mathematics and Its Applications (Soviet Series), 14, 1987. 